\title{
Multimodal image fusion in endovascular complex aortic aneurysm repair
}

Citation for published version (APA):

Karmann-Sailer, A-M. H. (2016). Multimodal image fusion in endovascular complex aortic aneurysm repair. [Doctoral Thesis, Maastricht University]. Datawyse / Universitaire Pers Maastricht. https://doi.org/10.26481/dis.20161214ak

Document status and date:

Published: 01/01/2016

DOI:

10.26481/dis.20161214ak

Document Version:

Publisher's PDF, also known as Version of record

\section{Please check the document version of this publication:}

- A submitted manuscript is the version of the article upon submission and before peer-review. There can be important differences between the submitted version and the official published version of record.

People interested in the research are advised to contact the author for the final version of the publication, or visit the DOI to the publisher's website.

- The final author version and the galley proof are versions of the publication after peer review.

- The final published version features the final layout of the paper including the volume, issue and page numbers.

Link to publication

\footnotetext{
General rights rights.

- You may freely distribute the URL identifying the publication in the public portal. please follow below link for the End User Agreement:

www.umlib.nl/taverne-license

Take down policy

If you believe that this document breaches copyright please contact us at:

repository@maastrichtuniversity.nl

providing details and we will investigate your claim.
}

Copyright and moral rights for the publications made accessible in the public portal are retained by the authors and/or other copyright owners and it is a condition of accessing publications that users recognise and abide by the legal requirements associated with these

- Users may download and print one copy of any publication from the public portal for the purpose of private study or research.

- You may not further distribute the material or use it for any profit-making activity or commercial gain

If the publication is distributed under the terms of Article $25 \mathrm{fa}$ of the Dutch Copyright Act, indicated by the "Taverne" license above, 


\section{Multimodal Image Fusion in Endovascular Complex Aortic Aneurysm Repair}

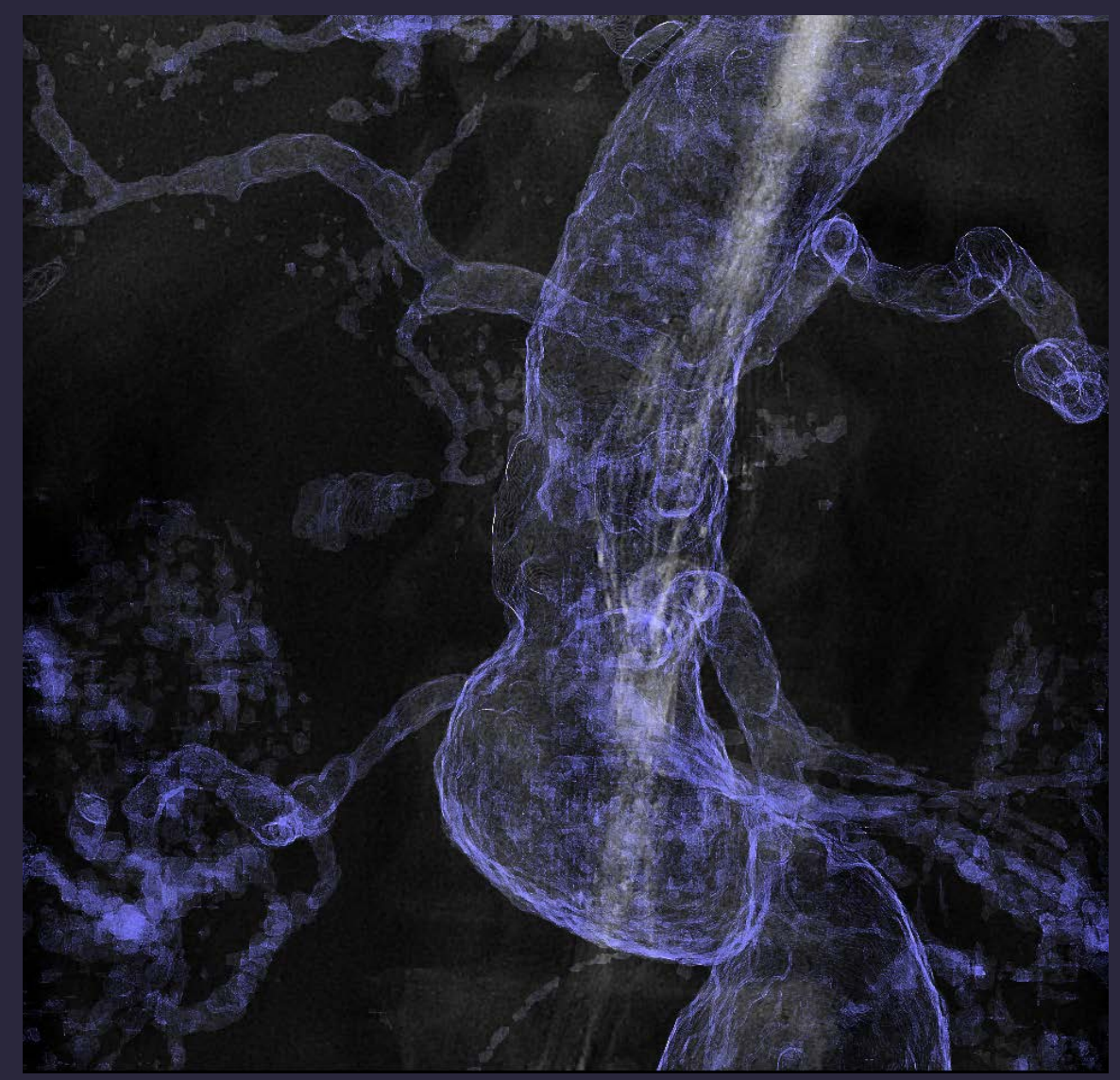

Anna-Margaretha Sailer 
Copyright of the individual chapters lies with the publisher of the journal listed at the beginning of each respective chapter. No part of this thesis may be reproduced in any form, by print, photocopy, digital file, internet, or any other means without written permission from the author. 


\title{
Multimodal Image Fusion in
}

\section{Endovascular Complex Aortic Aneurysm Repair}

\author{
PROEFSCHRIFT \\ ter verkrijging van de graad van doctor aan \\ de Universiteit Maastricht, \\ op gezag van de Rector Magnificus, \\ Prof. dr. Rianne M. Letschert \\ volgens het besluit van het College van Decanen, \\ in het openbaar te verdedigen \\ op woensdag 14 december 2016 om 12.00 uur
}

door

Anna-Margaretha Hedwig Karmann (geboren Sailer) 


\section{Promotores}

Prof. Dr. G.W.H. Schurink

Prof. Dr. M.W. de Haan

\section{Copromotor}

Dr. C.R.L.P.N. Jeukens

\section{Beoordelingscommissie}

Prof. Dr. R.J. van Oostenbrugge (voorzitter)

Prof. Dr. W.F. Buhre

Dr. J.A. van Herwaarden (Universitair Medisch Centrum, Utrecht)

Prof. Dr. P.A.M. Hofman

Prof. Dr. L.J. Schultze Kool (Radboud Universitair Medisch Centrum, Nijmegen) 


\section{CONTENTS}

Chapter 1 General Introduction

7

Chapter 2 CTA with fluoroscopy image fusion guidance in endovascular complex aortic aneurysm repair

Chapter 3 Quantification of respiratory movement of the aorta and side branches

Chapter 4 Radiation exposure of abdominal cone beam computed tomography

Chapter 5 Occupational radiation exposure during endovascular aortic repair

Chapter 6 Endovascular treatment of complex aortic aneurysms: Prevalence of acute kidney injury and effect on long-term renal function

Chapter 7 Fusion image guidance in endovascular peripheral artery interventions - a feasibility study

Chapter 8 General Discussion

Chapter 9 Valorization 105

Summary 109

Samenvatting 111

Acknowledgement 115

Curriculum Vitae

List of Publications

121 

CHAPTER

General Introduction 


\section{ENDOVASCULAR AORTIC ANEURYSM REPAIR}

Aortic aneurysms are defined as aortic segments with a $50 \%$ increase in diameter compared to the normal aorta. ${ }^{1}$ Aortic aneurysms affect between 2 and 8 percent of the western population with a generally higher prevalence in males, patients of advanced age and a localization in the abdominal aorta. ${ }^{2-4}$ Although pathogenesis of aneurysm formation and growth are not fully understood, aortic aneurysms have the habit to grow over time, significantly increasing the risk of rupture and eventual mortality. ${ }^{5-7}$ Surgical repair with open exposure of the aneurysm has successfully been performed since the 1950s by suturing a prosthetic graft proximally and distally into the adjacent aorta or iliac arteries. The high mortality rate of more than $4 \%$ associated with open aneurysm repair and a general trend towards minimally invasive surgery techniques led to the concept of delivering a covered stent graft endoluminally to the location of the aneurysm, effectively sealing off the aneurysm wall from systemic pressures and thereby preventing further aneurysm growth and rupture. ${ }^{8}$

In 1991, the first published report of stent graft implantation for abdominal aortic aneurysm in humans delivered scientific proof for the feasibility of endovascular aneurysm exclusion by delivering an endograft through small incisions in peripheral arteries. Since then three prospective randomized controlled trials on elective aortic repair have shown a manifest benefit of 30-day postoperative mortality in favor of endovascular treatment compared to open surgery. ${ }^{10-12}$ These results have been supported by data from large registries and one metaanalysis. ${ }^{13-15}$ For low risk patients, operative mortality rates between open surgery and endovascular repair were found similar. ${ }^{16}$ In 1994, Dake et al first reported the use of thoracic endovascular aortic repair (TEVAR) by introducing stent-grafts for the treatment of descending thoracic aortic aneurysms in a series of patients who were at high risk for open surgery. ${ }^{17}$ In the following 20 years, endovascular aortic repair has evolved at a rapid pace, targeting the treatment of more and more complex vascular pathology.

Aortic aneurysms are considered complex when the arterial anatomy does not provide a sufficient infrarenal or thoracic sealing zone. In consequence, the aortic stent graft needs to be extended above the level of relevant side branches such as the renal arteries, visceral arteries and supraaortic branches. In order to provide adequate graft sealing and, at the same time, preserve aortic side branch patency and blood supply to the depending organs, the main aortic branches arising from the affected part of the aorta are perioperatively catheterized and stented through customized fenestrations or branches in the aortic stent graft (FEVAR/BEVAR). ${ }^{18}$ Fenestrated and branched endovascular aortic repair (FEVAR/BEVAR) nowadays allows the treatment of complex aortic aneurysms such as juxta-and suprarenal aneurysms, thoraco-abdominal aneurysms and aortic arch aneurysms. 


\section{CHALLENGES OF ENDOVASCULAR REPAIR OF COMPLEX AORTIC ANEURYSMS}

Although standard endovascular repair proved favorable in perioperative survival, blood loss and shorter hospital stay compared to open surgery, EVAR relies on exposure to ionizing radiation and iodinated contrast medium. In addition, endovascular approaches face several challenges in complex aortic pathology. The endovascular repair of complex vascular anatomy is highly personalized and crucially depends on the quality and cooperation of different imaging modalities, with pre-operative computed tomography (CT) and intraoperative fluoroscopy being the most commonly used radiologic techniques. With increasing complexity of the treated pathology and the introduction of customized fenestrated and branched endovascular technology, the ability to precisely assess the three-dimensional (3D) architecture of the patient's aorta and its side branches before and during the procedure becomes crucial for a safe and effective endovascular repair. Multidetector computed tomography (MDCT) provides detailed 3D morphologic information of the aorta and arterial structure which allows for adequate preoperative patient selection, detailed procedure planning and patient-specific prosthesis sizing.

Intraoperative arterial imaging has historically been limited to two-dimensional (2D) angiography and fluoroscopy. The technical success of fenestrated and branched aneurysm repair relies substantially on the secure access to the side aortic branches. This requires correct positioning and deployment of the aortic stent graft at the right level and degree of rotation, atraumatic navigation into the target vessels through the fenestrations and branches as well as adequate stenting of the vessels.

A safe and effective execution of these complex procedures would ideally require intraoperative access to the entire 3D vascular architecture information of the MDCT in order to have constant knowledge about the position of the origins of the target vessel and aortic anatomy which would facilitate optimal viewing projections and 3D image guided navigation. With the current, conventional 2D fluoroscopy and angiography, guidewires and catheters are maneuvered "blindly" without visualization of the arterial anatomy or by road mapping based on vascular architecture visualization obtained from administration of iodinated contrast media. Different projections are usually mandatory for different target vessels which typically requires repeated contrast administration for new roadmaps in different working projections.

The technical complexity of fenestrated and branched procedures on one hand and the limited intraoperative vascular information on the other contribute to a substantial increase in procedure time, radiation exposure and iodinated contrast media in endovascular complex aneurysm repair. ${ }^{19,20}$ Higher radiation exposure increases the risk of deterministic injuries and induction of stochastic carcinogenic effects in patients, endovascular surgeons, interventional radiologists and other staff members involved in 
the procedures. ${ }^{21}$ Published evidence suggests the dose-dependent nephrotoxic agency of iodinated contrast media and its contribution to acute and chronic kidney injury, although this effect has not yet been fully studied for intra-arterial contrast media administration during endovascular complex aneurysm repair. ${ }^{22-24}$

\section{HYBRID OPERATING ROOMS}

Real-time fluoroscopic imaging has been used for medical diagnostic purposes shortly after the discovery of "a new kind of rays" by Wilhelm Conrad Roentgen 8 November 1995, the Röntgenstrahlen or X-rays. ${ }^{25}$ First fluoroscopes consisted of an X-ray tube, fluorescent material (in the early years barium platinocyanide) and a fluorescent screen. The fluorescent material emitted visible light in response to X-rays and facilitated realtime display on a screen put between the patient and the examiner. Despite ongoing technical improvements in the following years, light output was low and required darkness adaptation of the examiner's eye prior to the examination together with high burden of radiation. ${ }^{25,26}$ A breakthrough in fluoroscopic imaging came with the introduction of the X-ray image intensifier (II) in the late 1940s. ${ }^{26}$ This technology implanted the conversion of X-rays to light to electrons and provided output of nonlimiting brightness and smaller image sizes. Optical coupling of the output image to a television (TV) system facilitated real-time video image display. Further improvements in image quality by adapting resolution and bandwidth of the TV system developed throughout the following years.

In the late 1990s, flat panel X-ray detectors (FPD) were introduced and continuously replaced the standard II/TV systems used on conventional fluoroscopy C-arm units. ${ }^{26}$ FPD relied on new technology that converted X-ray into light for signal acquisition and digitalization, thereby permitting high exposure interventional procedures without overheating of the intensifier and facilitating detailed image display on large digital screens. ${ }^{27}$ The application of FPD has furthermore provided the ability to acquire and display intraoperatively three dimensional (3D) images using rotational fluoroscopy. Cone-beam computed tomography (CBCT) acquired by a FPD is an advanced imaging technology which provides intraoperative volumetric "CT-like" images in multiple viewing planes. ${ }^{28}$ State of the art hybrid operating rooms with FPD and CBCT technology are now commercially available by various manufacturers and have been introduced to Maastricht University Medical Centre in 2012. 


\section{MULTIMODAL IMAGE FUSION}

With the development of hybrid operating rooms, the technology of multimodal image fusion became available for endovascular treatment. Multimodal image fusion is the overlay of two or more imaging datasets as a co-display. ${ }^{29,30}$ For endovascular repair, the detailed vascular 3D data from the diagnostic MDCT can be fused to the live fluoroscopy image serving as an arterial roadmap of the vascular architecture making the full diagnostic data available to the operator during endovascular intervention. 3D coregistration of the MDCT dataset and live fluoroscopy can be performed by means of an intraoperatively performed $\mathrm{CBCT}^{31}$ Using commercially available software, corresponding anatomic landmarks such as bony structures or vessel wall calcifications on both the MDCT and CBCT datasets are automatically or manually matched and adjusted. This aligns the MDCT volume to the exact 3D coordinate space of the C-arm and live fluoroscopy image. Following the multimodality co-registration, the MDCT image is superimposed on the dynamic fluoroscopic image and will follow any 3D rotation of the $\mathrm{C}$-arm and table movement in real-time. With volume rendering adjustments or sections, the arterial structure of the MDCT can be made visible as a vascular roadmap without over-projection of bones or soft tissue structures. Multimodal image fusion of MDCT and live fluoroscopy may improve vascular visualization during endovascular aneurysm repair procedures which may in turn allow physicians to efficiently and precisely navigate guidewires and devices through the background MDCT roadmap. First studies suggest that multimodal image fusion in endovascular aortic repair may provide potential benefits by decreasing administration of iodinated contrast media used for conventional angiography road-mapping. ${ }^{28,32}$

\section{AIMS AND OUTLINE OF THIS THESIS}

The aim of this thesis was to evaluate the benefit and limitations of multimodal image fusion in endovascular complex aortic aneurysm repair. The thesis further strives to provide advanced knowledge on the procedure-specific patient and staff radiation exposure as well as insights to potential factors that may drive postoperative renal function decline after fenestrated and branched endovascular complex aortic aneurysm repair.

In Chapter 2, the application of multimodal image fusion in endovascular complex aortic aneurysm repair and its effect on procedural iodinate contrast media usage, procedure time and fluoroscopy time are evaluated. The main limitations of fusion image accuracy are assessed. 
In Chapter 3, the respiratory movement of the aorta and its side branches is investigated and quantified, which has been identified as the main contributor to overlay inaccuracy in chapter two.

In Chapter 4, the radiation exposure to patients undergoing СВCT for multimodal image fusion in endovascular complex aortic aneurysm repair is investigated.

In Chapter 5, the radiation exposure to physicians and operating room personnel during standard and complex endovascular aortic repair is studied.

In Chapter 6, risk factors for acute kidney injury and long term renal function deterioration in patients with complex aortic aneurysms undergoing endovascular repair are evaluated.

In Chapter 7, the feasibility of applying multimodal image fusion to other image guided procedures is investigated in the concrete example of magnetic resonance angiography with fluoroscopy image fusion in endovascular peripheral artery interventions.

In Chapter 8, the results of this thesis are summarized and discussed and prospects for future research are given.

\section{REFERENCES}

1. Johnston KW, Rutherford RB, Tilson MD, Shah DM, Hollier L, Stanley JC. Suggested standards for reporting on arterial aneurysms. Ad Hoc Committee on Reporting Standards, Society for Vascular Surgery and North American Chapter, International Society for Cardiovascular Surgery. J Vasc Surg. 1991;13:452- 458.

2. Bengtsson $\mathrm{H}$, Bergqvist $\mathrm{D}$, Sternby $\mathrm{NH}$. Increasing prevalence of abdominal aortic aneurysms: a necropsy study. Eur J Surg. 1992;158:19-23.

3. McFarlane MJ. The epidemiologic necropsy for abdominal aortic aneurysm. JAMA. 1991; 265:2085-2088.

4. Singh K, Bonaa KH, Jacobsen BK, Bjork L, Solberg S. Prevalence of and risk factors for abdominal aortic aneurysms in a population-based study: the Tromso Study. Am J Epidemiol. 2001;154:236 -244.

5. Hellenthal FA, Buurman WA, Wodzig WK, Schurink GW. Biomarkers of AAA progression. Part 1: extracellular matrix degeneration. Nat Rev Cardiol. 2009;6(7):464-74.

6. Hellenthal FA, Buurman WA, Wodzig WK, Schurink GW. Biomarkers of abdominal aortic aneurysm progression. Part 2: inflammation. Nat Rev Cardiol. 2009;6(8):543-52.

7. GBD 2013 Mortality and Causes of Death Collaborators. Global, regional, and national age-sex specific allcause and cause-specific mortality for 240 causes of death, 1990-2013: a systematic analysis for the Global Burden of Disease Study 2013. Lancet. 2015;385(9963):117-71.

8. Eliason JL, Upchurch GR Jr. Endovascular abdominal aortic aneurysm repair. Circulation. 2008;117(13):1738-44.

9. Parodi JC, Palmaz JC, Barone HD. Transfemoral intraluminal graft implantation for abdominal aortic aneurysms. Ann Vasc Surg.1991;5:491- 499.

10. EVAR trial participants. Endovascular aneurysm repair versus open repair in patients with abdominal aortic aneurysm (EVAR trial 1): randomised controlled trial. Lancet. 2005;365:2179-2186. 
11. Blankensteijn JD, de Jong SE, Prinssen M, van der Ham AC, Buth J, van Sterkenburg SM, Verhagen HJ, Buskens E, Grobbee DE; Dutch Randomized Endovascular Aneurysm Management (DREAM) Trial Group. Dutch Randomized Endovascular Aneurysm Management (DREAM) Trial Group. Two-year outcomes after conventional or endovascular repair of abdominal aortic aneurysms. N Engl J Med. 2005;352:2398-2405.

12. Lederle FA, Freischlag JA, Kyriakides TC, Padberg FT Jr, Matsumura JS, Kohler TR, Lin PH, Jean-Claude JM, Cikrit DF, Swanson KM, Peduzzi PN; Open Versus Endovascular Repair (OVER) Veterans Affairs Cooperative Study Group. Outcomes following endovascular vs open repair of abdominal aortic aneurysm: a randomized trial. JAMA. 2009;302(14):1535-42.

13. Schermerhorn ML, O'Malley AJ, Jhaveri A, Cotterill P, Pomposelli F, Landon BE. Endovascular vs. open repair of abdominal aortic aneurysms in the Medicare population. N Engl J Med. 2008;358:464-474.

14. Harris PL, Vallabhaneni SR, Desgranges P, Becquemin JP, van Marrewijk C, Laheij RJ. Incidence and risk factors of late rupture, conversion, and death after endovascular repair of infrarenal aortic aneurysms: the EUROSTAR experience. European Collaborators on stent/graft techniques for aortic aneurysm repair. J Vasc Surg. 2000;32:739-749.

15. Lovegrove RE, Javid M, Magee TR, Galland RB. A meta-analysis of 21,178 patients undergoing open or endovascular repair of abdominal aortic aneurysm. Br J Surg. 2008;95:677-684.

16. Becquemin JP. The ACE trial: a randomized comparison of open versus endovascular repair in good risk patients with abdominal aortic aneurysm. J Vasc Surg. 2009;50:222-224.

17. Dake MD, Miller DC, Semba CP, Mitchell RS, Walker PJ, Liddell RP. Transluminal placement of endovascular stent-grafts for the treatment of descending thoracic aortic aneurysms. N Engl J Med. 1994;331:1729-34.

18. Browne TF, Hartley D, Purchas S, Rosenberg M, Schie G Van, Lawrence-Brown M. A fenestrated covered suprarenal aortic stent. Eur J Vasc Endovasc Surg. 1999;18:445-449.

19. Kirkwood ML, Arbique GM, Guild JB, Timaran C, Chung J, Anderson JA, Valentine RJ. Surgeon education decreases radiation dose in complex endovascular procedures and improves patient safety. J Vasc Surg. 2013 Sep;58(3):715-21.

20. McNally MM, Scali ST, Feezor RJ, Neal D, Huber TS, Beck AW. Three-dimensional fusion computed tomography decreases radiation exposure, procedure time, and contrast use during fenestrated endovascular aortic repair. J Vasc Surg. 2015 Feb;61(2):309-16.

21. The 2007 Recommendations of the International Commission on Radiological Protection. ICRP publication 103. Ann ICRP 37:1-33.

22. Solomon R, Dumouchel W. Contrast media and nephropathy: findings from systematic analysis and Food and Drug Administration reports of adverse effects. Invest Radiol 2006;41: 651e60.

23. Kane GC, Doyle BJ, LermanA, Barsness GW, Best PJ, Rihal CS. Ultra-Low Contrast Volumes Reduce Rates of Contrast-Induced Nephropathy in Patients With Chronic Kidney Disease Undergoing Coronary Angiography J Am Coll Cardiol. 2008;51(1):89-90

24. Davenport MS, Cohan RH, Khalatbari S, Ellis JH. The challenges in assessing contrast-induced nephropathy: where are we now? AJR Am J Roentgenol. 2014; 202:784-9.

25. Eisenberg RL (1992) Radiology, an illustrated history. Chapter 10: dark adaptation and image intensification. Mosby Year Book, St. Louis, pp 149-55.

26. Krohmer JS. Radiography and fluoroscopy, 1920 to the present. RadioGraphics 1989; 9:1129-1153.

27. Schueler BA. The AAPM/RSNA physics tutorial for residents: general overview of fluoroscopic imaging. Radiographics. 2000 Jul-Aug;20(4):1115-26. 


\section{CHAPTER 1}

28. Dijkstra ML, Eagleton MJ, Greenberg RK, Mastracci T, Hernandez A. Intraoperative C-arm cone-beam computed tomography in fenestrated/branched aortic endografting. J Vasc Surg. 2011 Mar;53(3):583-90.

29. Garcia JA, Bhakta S, Kay J, Chan KC, Wink O, Ruijters D, Carroll JD. On-line multi-slice computed tomography interactive overlay with conventional X-ray: a new and advanced imaging fusion concept. Int J Cardiol. 2009, 133:e101-e105.

30. Lin CJ, Blanc R, Clarencon F, Piotin M, Spelle L, Guillermic J, Moret J. Overlying fluoroscopy and preacquired CT angiography for roadmapping in cerebral angiography. AJNR Am J Neuroradiol. 2010;31:494-495.

31. Abi-Jaoudeh N, Kruecker J, Kadoury S, Kobeiter H, Venkatesan AM, Levy E, Wood BJ. Multimodality image fusion-guided procedures: technique, accuracy, and applications. Cardiovasc Intervent Radiol. 2012;35:986-98.

32. Kobeiter H, Nahum J, Becquemin JP. Zero-contrast thoracic endovascular aortic repair using image fusion. Circulation. 2011 Sep 13;124(11):e280-2. 


\section{CHAPTER}

CTA with fluoroscopy image fusion guidance in endovascular complex aortic aneurysm repair

A.M. Sailer

M.W. de Haan

A.G. Peppelenbosch

M.J. Jacobs

J.E. Wildberger

G.W.H. Schurink

European Journal of Vascular and Endovascular Surgery 2014 Apr;47(4):349-56 


\section{ABSTRACT}

Objective: To evaluate the effect of intraoperative guidance by means of live fluoroscopy image fusion with computed tomography angiography (CTA) on iodinated contrast material volume, procedure time, and fluoroscopy time in endovascular thoracoabdominal aortic repair.

Methods: CTA with fluoroscopy image fusion road-mapping was prospectively evaluated in patients with complex aortic aneurysms who underwent fenestrated and/or branched endovascular repair (FEVAR/BEVAR). Total iodinated contrast material volume, overall procedure time, and fluoroscopy time were compared between the fusion group $(n=31)$ and case controls $(n=31)$. Reasons for potential fusion image inaccuracy were analyzed.

Results: Fusion imaging was feasible in all patients. Fusion image road-mapping was used for navigation and positioning of the devices and catheter guidance during access to target vessels. lodinated contrast material volume and procedure time were significantly lower in the fusion group than in case controls (159 mL [95\% Cl: $132-186 \mathrm{~mL}] \mathrm{vs.} 199 \mathrm{~mL}$ [95\% Cl: $170-229 \mathrm{~mL}$ ], $p=.037$ and 5.2 hours [95\% Cl: $4.5-5.9$ hours] vs. 6.3 hours (95\% $\mathrm{Cl}: 5.4-7.2$ hours), $\mathrm{p}=.022$ ). No significant differences in fluoroscopy time were observed ( $p=.38$ ). Respiration-related vessel displacement, vessel elongation, and displacement by stiff devices as well as patient movement were identified as reasons for fusion image inaccuracy.

Conclusion: Image fusion guidance provides added value in complex endovascular interventions. The technology significantly reduces iodinated contrast material dose and procedure time. 


\subsection{INTRODUCTION}

Multimodality image fusion guidance is a new alternative to conventional angiogram road-mapping in endovascular interventions. The technology follows two principles: ${ }^{1}$ (a) to coregister and fuse pre-acquired image datasets (e.g. computed tomography angiography [CTA] or magnetic resonance angiography [MRA]) with another dynamic imaging modality, commonly fluoroscopy; (b) to overlay real-time catheter and guidewire movements to the pre-acquired background dataset. Rigid co-registration is performed by means of a C-arm cone beam computed tomography (CBCT) system, allowing the preacquired dataset to follow any rotation of the C-arm and table movement. Recently, fusion image road-mapping has been evaluated for cardiologic interventions and in endovascular surgery. ${ }^{2-6}$ Nevertheless, broad application of this technique for routine practice and evaluation of added value and limitations has yet to happen. The purpose of this work was to evaluate the effect of realtime fluoroscopy with CTA image fusion guidance on iodinated contrast material usage, procedure time, and fluoroscopy time in branched and fenestrated repair of complex aortic aneurysms. We further aimed to analyze and quantify limitations of fusion road-map accuracy.

\subsection{MATERIALS AND METHODS}

Image fusion technology was prospectively evaluated for intraprocedural road-mapping in 31 patients with complex aortic aneurysms who underwent fenestrated and/or branched endovascular repair (FEVAR/BEVAR) between March 2012 and September 2013. The study was approved by the institutional ethics committee and the requirement for informed consent was waived by the IRB. Prior to the intervention, all patients had undergone outpatient diagnostic CTA scans on a second-generation dual-source CT scanner (Somatom Definition, Siemens Healthcare, Forchheim, Germany) for measurements and planning of the custom-made endografts. The CTA scans were carried out in dual-energy settings, allowing for dedicated post-processing, including automated bone removal. The endovascular procedure was performed in our hybrid operating theater. Just before surgical vascular access was obtained, an abdominal non contrastenhanced CBCT was performed by rotational movement of the C-arm covering a 180degree circular trajectory (Allura Xper FD20, Philips Healthcare, Best, The Netherlands). Standard parameters for CBCT were $123 \mathrm{kV}$ and additional filtration of $0.9 \mathrm{~mm}$ of copper plus $1 \mathrm{~mm}$ of aluminum. The volumetric dataset of a total of 316 images was automatically transferred to a 3D workstation (Philips Allura XtraVision 8.3) and coregistered to patients' preinterventional diagnostic CTA images. Arterial vessel wall calcifications and bone borders were used as landmarks. During the intervention, the fusion road-map was presented in real-time on a screen next to conventional fluoroscopy. 
Fusion image road-mapping was used for navigation and positioning of the stent graft. Before deployment, the accuracy of the fusion road-map and correct position of the stent graft were evaluated by conventional angiography. For catheterization of fenestrations, branches and target vessel, optimal C-arm projection angles were identified on the fusion road-map and access was obtained using fusion guidance. After catheterization, the correct position was tested by catheter injection.
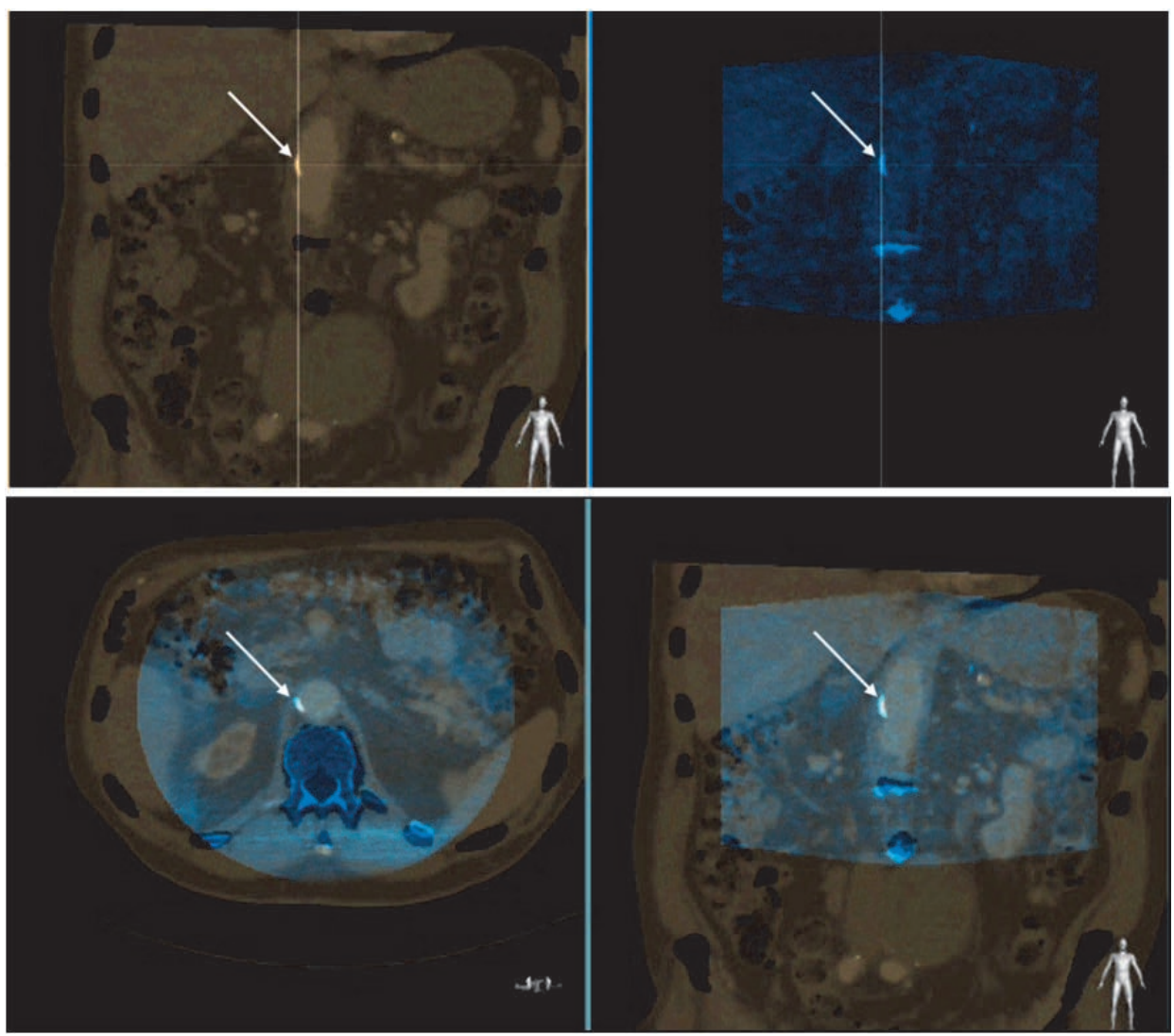

Figure 1. Three dimensional co-registration of volumetric pre-acquired computed tomography angiography (CTA) dataset (grey) with cone beam computed tomography (CBCT) dataset (blue). Top row: Aortic wall calcifications (white arrows) on CBCT and CTA datasets are selected as landmarks and marked with the crosshair. Bottom row: The fused dataset is further adjusted in three dimensions by scrolling through the dataset and aligning bone borders and other calcifications (sagittal plane not shown).

\section{Case-controls}

In our clinic, more than 150 FEVAR and BEVAR procedures have been performed since 2006. These procedures were performed by the same vascular surgeon together with the same interventional radiologist (last and second author). 
Patients undergoing FEVAR/BEVAR between March 2012 and September 2013 were consecutively included in the fusion guidance group. Due to maintenance/software updates, the fusion technology was not available in October 2012 and between April and May 2013; procedures during this period were included in the caseecontrol group. Cases and controls were collected from our historical pool of FEVAR/BEVAR procedures without fusion guidance and limited to the period between 2010 and 2013. Cases and controls were technically comparable in procedure complexity expressed in type of aneurysm (type of TAAA according to Crawford classification, juxtarenal AAA, and one iliac aneurysm), number of fenestrations, and branches as well as presence or absence of a bifurcated endovascular graft. Where more than one case control matched the fusion case, the more recent procedure was included.

\section{Outcome parameters}

The radiation dose of CBCT in terms of dose-area product (DAP, in $\mathrm{mGym}^{2}$ ) was registred. The total volume of procedural iodinated contrast material (iopromide, $300 \mathrm{mg} / \mathrm{mL}$; Ultravist, Bayer HealthCare, Berlin) and overall procedure time were registered and compared with data from the case-controls. For the last 22 procedures, fluoroscopy time was also registered and compared with available case-control data. Reasons for potential fusion road-map inaccuracies were analyzed and quantified by the multidisciplinary team.

\section{Statistical analysis}

For continuous variables, the t test for independent samples or the Wilcoxon test was used for normally and non normally distributed data respectively (SPSS statistics 20.0, Chicago, IL, USA). Two sided $p<.05$ were considered significant. Quantitative values are presented by means and $95 \%$ confidence intervals.

\subsection{RESULTS}

The fusion group consisted of 26 men and five women, with a mean age of $74 \pm 6$ years. The demographics of the fusion $(n=31)$ and caseecontrol groups $(n=31)$ were similar. An accurate caseecontrol match for every fusion patient was retrieved on the basis of aneurysm and endograft characteristics. Detailed information on aneurysm type and endograft design for fusion and control patients is shown in Table 1. Mean radiation dose of CBCT was $1.43 \mathrm{mGym}^{2}$ (95\% Cl: 1.20 - $1.66 \mathrm{mGym}^{2}$ ). Image co-registration and fusion guidance were feasible in all procedures. Coregistration of the CBCT to the CTA dataset is shown in Figure 1. Figures 2 and 3 demonstrate fusion guidance during the FEVAR and BEVAR procedures. 
Table 1. Aneurysm characteristics and FEVAR/BEVAR design in the fusion and caseecontrol groups.

\begin{tabular}{|c|c|c|c|c|c|c|c|}
\hline \multicolumn{4}{|c|}{ Fusion group } & \multicolumn{4}{|c|}{ Case Control group } \\
\hline $\begin{array}{l}\text { Patient } \\
\text { ID }\end{array}$ & type aneurysm & $\begin{array}{l}\text { no. of fenestrations }(\mathrm{F}) / \\
\text { branches (B) }\end{array}$ & $\begin{array}{c}\text { bifurcated } \\
\text { endovascular graft }\end{array}$ & $\begin{array}{l}\text { Patient } \\
\text { ID }\end{array}$ & type aneurysm & $\begin{array}{c}\text { no. of fenestrations }(\mathbf{F}) / \\
\text { branches }(B)\end{array}$ & $\begin{array}{c}\text { bifurcated } \\
\text { endovascular graft }\end{array}$ \\
\hline 1 & TAAA I & $4 \mathrm{~B}$ & no & 32 & TAAA I & $4 \mathrm{~B}$ & no \\
\hline 2 & TAAA III & $3 \mathrm{~F} / 1 \mathrm{~B}$ & no & 33 & TAAA IV & $4 \mathrm{~F}$ & no \\
\hline 4 & juxtarenal AAA & $4 \mathrm{~F}$ & yes & 35 & TAAA IV & $3 \mathrm{~F} / 1 \mathrm{~B}$ & yes \\
\hline 5 & TAAA I & $2 \mathrm{~F}$ & no & 36 & TAAA I & $2 \mathrm{~F}$ & no \\
\hline 6 & TAAA III & $4 \mathrm{~B}$ & no & 37 & TAAA IV & $4 \mathrm{~B}$ & no \\
\hline 7 & TAAA III & $4 \mathrm{~F}$ & no & 38 & TAAA IV & $4 \mathrm{~F}$ & no \\
\hline 11 & juxtarenal AAA & $3 \mathrm{~F}$ & yes & 42 & juxtarenal AAA & $3 \mathrm{~F}$ & yes \\
\hline 12 & TAAA II & $4 \mathrm{~B}$ & no & 43 & TAAA II & $4 \mathrm{~B}$ & yes \\
\hline 13 & TAAA II & $4 \mathrm{~B}$ & yes & 44 & TAAA II & $4 \mathrm{~B}$ & yes \\
\hline 14 & TAAA III & $4 \mathrm{~B}$ & yes & 45 & TAAA III & $1 \mathrm{~F} / 3 \mathrm{~B}$ & no \\
\hline 15 & TAAA III & $4 \mathrm{~B}$ & no & 46 & TAAA II & $4 \mathrm{~B}$ & no \\
\hline 16 & TAAA IV & $3 \mathrm{~F}$ & yes & 47 & TAAA IV & $4 \mathrm{~B}$ & yes \\
\hline 17 & juxtarenal AAA & $3 \mathrm{~F}$ & yes & 48 & juxtarenal AAA & $3 \mathrm{~F}$ & yes \\
\hline 18 & TAAA I & $4 \mathrm{~F}$ & no & 49 & TAAA I & $4 \mathrm{~B}$ & no \\
\hline 19 & juxtarenal AAA & $3 \mathrm{~F}$ & yes & 50 & juxtarenal AAA & $3 \mathrm{~F}$ & yes \\
\hline 25 & iliac aneurysm & $1 \mathrm{~B}$ & yes & 56 & iliac aneurysm & $1 \mathrm{~B}$ & yes \\
\hline 26 & juxtarenal AAA & $3 \mathrm{~F}$ & yes & 57 & juxtarenal AAA & $3 \mathrm{~F}$ & yes \\
\hline 27 & TAAA III & $3 \mathrm{~F} / 1 \mathrm{~B}$ & yes & 58 & TAAA III & $1 F / 3 B$ & yes \\
\hline 28 & juxtarenal AAA & $1 \mathrm{~F}$ & no & 59 & juxtarenal AAA & $2 \mathrm{~F}$ & no \\
\hline 29 & juxtarenal AAA & $3 \mathrm{~F}$ & yes & 60 & juxtarenal AAA & $3 \mathrm{~F}$ & yes \\
\hline 30 & TAAA V & $2 \mathrm{~F} / 2 \mathrm{~B}$ & no & 61 & TAAA IV & $4 \mathrm{~F}$ & no \\
\hline 31 & TAAA III & $2 \mathrm{~F} / 2 \mathrm{~B}$ & no & 62 & juxtarenal AAA & $2 \mathrm{~F}$ & no \\
\hline \multicolumn{2}{|c|}{ Total number F / B: } & $61 \mathrm{~F} / 51 \mathrm{~B}$ & & \multicolumn{2}{|c|}{ Total number F / B: } & $56 \mathrm{~F} / 51 \mathrm{~B}$ & \\
\hline
\end{tabular}

All variables were normally distributed. The mean total procedure time in the fusion group was 5.2 hours ( $95 \% \mathrm{Cl}: 4.5$ - 5.9 hours), which was significantly lower than the control group (6.3 hours, $95 \% \mathrm{Cl}: 5.4-7.2$ hours, $p=.022$ ). The mean procedural iodinated contrast material dose in the fusion group was $159 \mathrm{~mL}(95 \% \mathrm{Cl}: 132-186 \mathrm{~mL})$ versus $199 \mathrm{~mL}(95 \% \mathrm{Cl}: 170-229 \mathrm{~mL})$ in the control group. This difference was also significant $(p=.037)$. Concerning fluoroscopy time, complete data could be retrieved for 11 pairs of fusion and case-control procedures. The average fluoroscopy time of the fusion group (50 minutes and 26 seconds, $95 \% \mathrm{Cl}: 30: 33$ - 70:18) and case-control group (59 minutes and 29 seconds, 95\% Cl: 40:40 - 78:19) were not significantly different ( $p=$ 
.38). Table 2 provides a detailed overview of the objective outcome parameters of the fusion and case-control FEVAR/BEVAR procedures.

\section{Overlay inaccuracy}

To some extent, fusion image overlay inaccuracy was observed during all procedures and was grouped into three main categories.

1. Overlay inaccuracy due to differences between CTA and CBCT acquisition

The average time interval between acquisition of the diagnostic CTA and acquisition of the CBCT on the date of elective custom-made endograft repair was approximately 3 months. No overlay inaccuracies were observed due to these intervals. However, a mismatch between the pre-acquired CTA images and live fluoroscopy due to respirationrelated vessel displacement was observed in all patients. In our clinic, diagnostic CTA scans are performed during breath-hold inspiration: a condition which is not typical for general anesthesia with low tidal volume ventilation. Respiration-related vessel mismatch was mainly observed at the level of the aortic arch and peripheral segments of the visceral branches. In Figure 3, the mismatch between the tip of the guidewire and the CTA overlay road-map in a distal section of the renal and splenic arteries is visible.

2. Overlay inaccuracy due to rigid co-registration

Straightening and deformation of the vessel tree by stiff guidewires and stiff introduction devices was observed in 20 cases, mainly at the level of tortuous iliac arteries and distal elongated descending thoracic aorta. As co-registration with the pre-acquired CTA was performed in a rigid, non-elastic manner, any deformation of the vessel tree resulted in noneradicable overlay inaccuracy during maneuver with stiff devices. Figure 4 visualizes vessel deformity during graft introduction resulting in temporary overlay inaccuracy of the non-elastic fusion road-map

\section{Overlay inaccuracy due to patient movement}

Any patient movement after CBCT acquisition resulted in overlay inaccuracy to the same extent and in the same direction as the original movement. As patients were under general anesthesia during the intervention, and $\mathrm{CBCT}$ was acquired immediately before vascular access, inaccuracy due to patient movements was only observed if patients were repositioned by the surgical team. In the two cases, overlay mismatch could be eradicated by manual readjustment of the fusion road-map. 


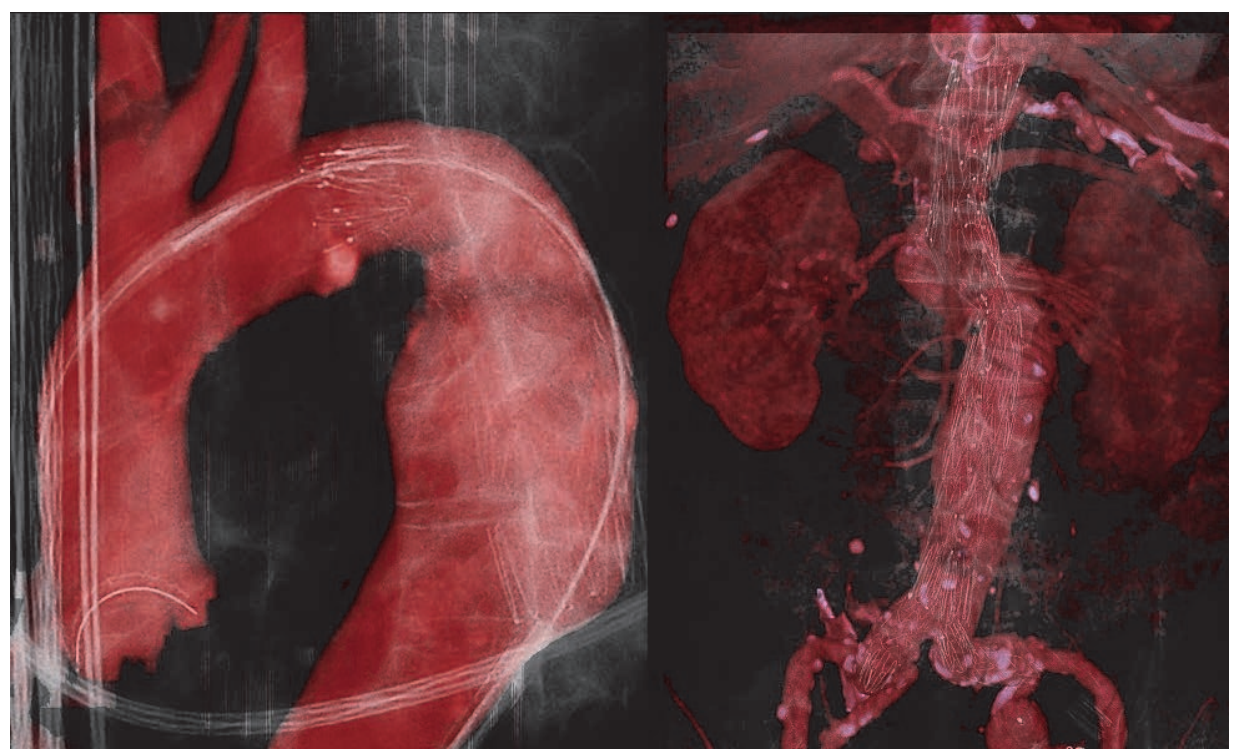

Figure 2. Stent graft placement based on fusion road-map after control for correct overlay position on conventional angiogram. Live fluoroscopy image with main body grafts (white) overlaid on pre-acquired computed tomography angiography background (red) in two different patients.
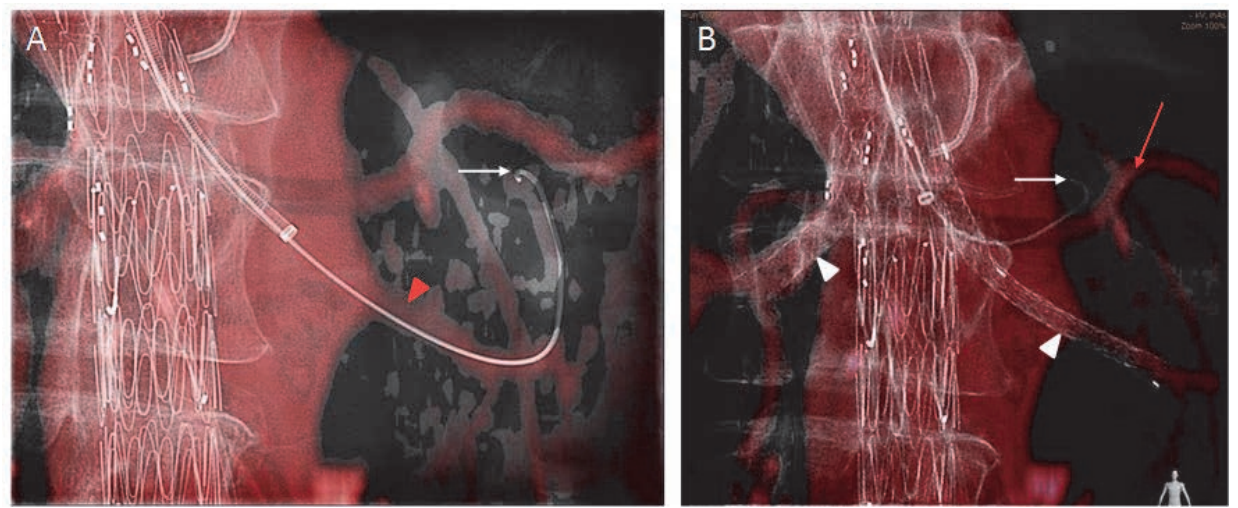

Figure 3. Fusion image guidance for left renal artery (A) and celiac trunk (B) catheterization from a transbrachial approach. Tip of the guidewire (white arrows) in the left renal artery (red arrow head) and splenic artery (red arrow) respectively. Covered stents in both renal arteries (white arrow heads).

\section{Complications}

A complication was observed during the procedure of patient no. 12. While achieving access to the target vessel through the branch of the right renal artery, the guidewire and catheter were incorrectly positioned in the right colic artery (RCA). With the single plane $\mathrm{C}$-arm in the anterior-posterior position and radiation protection shields covering the 
right flank, the superposition was neither detected on conventional angiogram nor on the fusion road-map. Deployment of the covered stent grafts obstructed the superior mesenteric artery distal to the RCA orifice with the need for surgical stent removal from the RCA and performance an iliac-superior mesenteric artery bypass. No major complications from the conversion have occurred. The patient has refused to undergo a second procedure connecting the renal and celiac branch to their target vessels.

Table 2. lodinated contrast volume, procedure time and fluoroscopy time in fusion and case-control procedures.

\begin{tabular}{|c|c|c|c|c|c|c|}
\hline \multirow{2}{*}{$\begin{array}{c}\text { ID } \\
\text { Fusion - Case Control }\end{array}$} & \multicolumn{2}{|c|}{$\begin{array}{l}\text { Procedure iodinated contrast } \\
\text { volume (in ml) }\end{array}$} & \multicolumn{2}{|c|}{$\begin{array}{l}\text { Total procedure time } \\
\text { (in hours) }\end{array}$} & \multicolumn{2}{|c|}{$\begin{array}{c}\text { Fluoroscopy time } \\
\text { (in minutes : seconds) }\end{array}$} \\
\hline & Fusion & Case Control & Fusion & Case Control & Fusion & Case control \\
\hline $1-32$ & 160 & na & 6 & 7.5 & & \\
\hline $2-33$ & 110 & 250 & 4.5 & 11 & & \\
\hline $3-34$ & 180 & 150 & 10 & 4.5 & & \\
\hline $4-35$ & 200 & 250 & 7.75 & 10.25 & & \\
\hline $5-36$ & 180 & 150 & 3.75 & 4 & & \\
\hline $6-37$ & 200 & 250 & 5.25 & 5.75 & & \\
\hline $7-38$ & 200 & 120 & 5.75 & 6 & & \\
\hline $8-39$ & 125 & 325 & 7 & 8 & & \\
\hline $9-40$ & 100 & 350 & 9 & 10 & & \\
\hline $10-41$ & 75 & 80 & 7 & 8 & $93: 41$ & na \\
\hline $11-42$ & 100 & 120 & 6 & 3 & na & $71: 02$ \\
\hline $12-43$ & conversion & 250 & conversion & 8 & conversion & na \\
\hline $13-44$ & 150 & 250 & 7.5 & 8 & $59: 58$ & na \\
\hline $14-45$ & 275 & 100 & 5 & 8.25 & $55: 00$ & $97: 00$ \\
\hline $15-46$ & 200 & 250 & 3 & 7.5 & $39: 29$ & na \\
\hline $16-47$ & 90 & 220 & 3.25 & 5.5 & $26: 42$ & na \\
\hline $17-48$ & 90 & 300 & 4.5 & 5 & $97: 34$ & $118: 24$ \\
\hline $18-49$ & 145 & 175 & 3.5 & 5 & $46: 03$ & na \\
\hline $19-50$ & 225 & 150 & 5.25 & 3 & $81: 12$ & $57: 38$ \\
\hline $20-51$ & 200 & 270 & 5 & 5.5 & $42: 21$ & $58: 00$ \\
\hline $21-52$ & 320 & 250 & 4.5 & 8.5 & $84: 29$ & $51: 00$ \\
\hline $22-53$ & 120 & 125 & 4.5 & 6 & $45: 41$ & $61: 39$ \\
\hline $23-54$ & 200 & 150 & 7 & 6.25 & $111: 13$ & na \\
\hline $24-55$ & 140 & 130 & 4.25 & 8 & $14: 45$ & $65: 53$ \\
\hline $25-56$ & 80 & 200 & 3.75 & 3,5 & $20: 53$ & $44: 38$ \\
\hline $26-57$ & 90 & 100 & 2.5 & 3 & $25: 56$ & 47:05 \\
\hline $27-58$ & 300 & 300 & 6 & 10.25 & $94: 47$ & na \\
\hline $28-59$ & 100 & 220 & 3 & 2 & $16: 19$ & $38: 52$ \\
\hline $29-60$ & 160 & 300 & 4.5 & 6 & $70: 32$ & $14: 13$ \\
\hline $30-61$ & 240 & 150 & 5 & 6 & $85: 47$ & na \\
\hline $31-62$ & 25 & 100 & 3 & 3 & $30: 09$ & na \\
\hline Mean & 159 & 199 & 5.2 & 6.3 & $50: 26$ & $59: 29$ \\
\hline Standard de viation & 71 & 78 & 1.8 & 2.4 & $29: 35$ & $28: 01$ \\
\hline
\end{tabular}

na $=$ data not available. Mean and Standard Deviation calculated only for pairs with complete data. 


\subsection{DISCUSSION}

The use of multimodality image fusion road-mapping in routine practice of complex endovascular aortic repair was evaluated. The data from the presented case-control study demonstrates that CTA with fluoroscopy fusion technology results in significant reduction in procedure time and iodinated contrast material volume. A trend towards lower procedural fluoroscopy time was observed. Significant reduction of contrast material volume $(p=.001)$ by means of fusion image road-mapping in complex EVAR has been described previously by Dijkstra et al. ${ }^{5}$ and Tacher et al. ${ }^{6}$ A single case of zerocontrast TEVAR using fusion has also been reported. ${ }^{4}$ Fenestrated and branched stent graft technology makes it possible to perform endovascular repair for complex aortic disease. Conventionally performed FEVAR/BEVAR is associated with a significant risk of adverse renal events due to the large volumes of contrast material used. ${ }^{7,8}$ Any effort and technology supporting a reduction in the volume of contrast material used is therefore highly relevant. Fusion image road-mapping promises significant benefits for vascular patients. However, there are some limitations of this technology and safety aspects that need to be discussed.

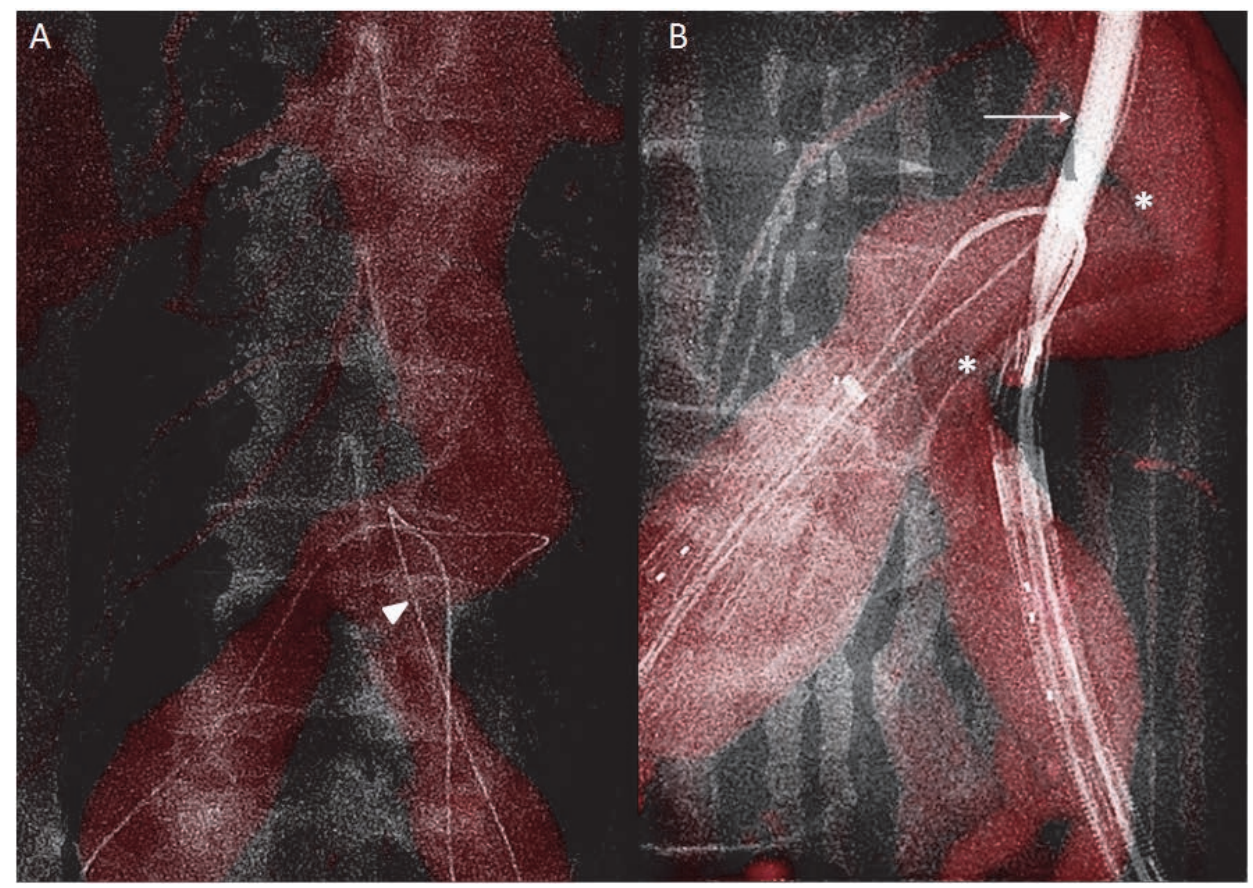

Figure 4. Fusion road-map overlay accuracy (A) with guide wires (white arrow head) precisely matching the vessel tree. Temporary local fusion overlay inaccuracy (B) during introduction of the stiff graft device (white arrow) at level of kinking elongated vessel tree (white asterisks) due to rigid image co-registration.

In our study, at all critical steps during the procedures, conventional angiograms were performed to confirm the correct position of the devices. This allowed us to get 
acquainted with fusion road-mapping and gain confidence with the new technology. In these procedures, contrast injections and procedure time were mainly reduced by more efficient target vessel catheterization under fusion road-map guidance. This provided a valuable benefit reflected in the results of our study. Nevertheless, this benefit is small compared with stent graft deployment used solely based on the fusion road-map. With growing experience, in the last procedure, the aortograms prior to successful main device deployment under fusion guidance were omitted. It is however important to note that the amount of contrast volume saved due to the omitted aortograms is of trivial importance compared with the potential complication of a misplaced fenestrated device. In order to rely fully on fusion road-mapping, practical experience and precise knowledge of the limitations and accuracy errors of this technology are of critical importance. Inaccuracies of the overlay road-map were observed to some extent in all procedures, with respiration-related vessel displacement and temporary straightening of elongated vessels during maneuver with stiff devices being the most frequent. However, these inaccuracies may not hamper use of the fusion road-map provided the error and its range become predicable. Inaccuracies in locating peripheral segments of vessels due to organ movement corresponding to respiration-related diaphragm movement do not limit the use of the fusion road-map as long as the origin of the target vessels does not change position. In the fusion roadmap accuracy analysis during EVAR from Fukuda et al., ${ }^{10}$ the mean error of renal artery origin overlay image was $2 \pm 2.5 \mathrm{~mm}$, with a range from 0 to 7 $\mathrm{mm}$. Further precise analyses of anatomy, respiration, and related overlay error are necessary to provide quantified predictions of overlay accuracy and expected extent of possible displacement. With growing experience and trust, further significant reductions of iodinated contrast volume and radiation exposure is to be expected.

There are limitations due to our study design. In the setting of advanced endovascular repair, procedure complexity and therefore procedure time, iodinated contrast volume and radiation dose are influenced by stent graft complexity. A case-control design with matching pairs based on stent graft complexity was therefore chosen. Controls were retrieved from a large pool of primarily historical procedures. This implies risk of bias in control selection as well as bias due to differences in operator experience. Controls had to most accurately technically match the fusion procedures; in the fusion group, the total number of fenestrations was slightly higher than the control group (Table 1). When there was more than one matching control, controls were prioritized by procedure date and only included procedures after 2010 aiming to minimize effects of experience level on our results. In this clinic, more than 150 FEVAR and BEVAR procedures have been performed since 2006, all carried out by the same two specialists. Assuming that the learning curve after 2010 might already have flattened, experience gain during the procedures included in this study was expected to have minor influence on the significance of our results. Since 2010, there have been no changes in operation protocol and time registration that could have influenced our results. However, prospective 
randomized controlled studies evaluating fusion guidance are lacking but are needed. Furthermore, in future studies, radiation exposure should be focused. Multimodality image co-registration is possible by means of performance of a C-arm CBCT system. With the surgical team, usually outside the operation theater during acquisition, CBCT does not result in additional radiation exposure for the medical staff. However, patients undergoing CBCT are exposed to an extra load of radiation, in our study in the range between 0.5 and $2.6 \mathrm{mGym}^{2}$, depending on body length and weight. The total DAP of fenestrated and branched endovascular aneurysm repair procedures has been described in the range of $1-78 \mathrm{mGym}^{2}{ }^{9}$. The case control pairs were not matched for body characteristics. Procedural DAP is influenced by patients' body mass index and further non-comparable between all procedures due to a software update of our hybrid fluoroscopy unit intended to shift radiation dose to a lower level. Considering this, the protocol was altered to compare fluoroscopy time, which is not influenced by body size and fluoroscopy units' filters but indirectly reflects radiation exposure. Mean fluoroscopy time was on average 9 minutes lower in the fusion group. However, complete data could only be provided for 11 pairs for this variable. This limitation might have resulted in this observation not reaching statistical significance. Nevertheless, any reduction in fluoroscopy time is still relevant as it results in effective radiation dose reduction for the medical staff. A significant reduction of fluoroscopy time by use of fusion road-mapping might equalize patients' total procedural radiation exposure or even be decreased by fusion technology. In conclusion, further research evaluating influence of fusion guidance on fluoroscopy time and procedural radiation dose is needed.

There was one complication in the group of fusion patients. Due to superposition of vessels on the single plane view, catheter malposition was not recognized. In this respect, fusion road-map did not show superiority in terms of safety compared with conventional angiography. As volumetric CTA datasets are registered, any further improvement of the technology to provide real-time threedimensional views would be beneficial and could address these actual limitations of two-dimensional fusion roadmapping.

Technical requirements for fusion guidance are a CBCT feasible fluoroscopy unit as well as dataset co-registration software. Flat panel detectors that can acquire rotational 3D images, such as CBCT, have begun to replace conventional fluoroscopy units and coregistration software for image fusion is commercially available from different vendors. In our experience, time for CBCT acquisition was approximately 5 minutes and image coregistration was carried out while further preparations of the patient took place. Actual time loss for fusion set up was therefore negligible. For fusion guidance, semi-automated or automated removal of bones and other radiodense nonvascular structures should be performed on CTA datasets to avoid over-projection on the vessel tree overlay image.

In conclusion, fusion image guidance provided added value for FEVAR/BEVAR procedures in terms of procedure time and contrast volume reduction. Further application of this 
new technology to less complex but high volume routine procedures, for example peripheral artery interventions might alter experience and further enhance procedural safety, for patients and surgeons.

\section{REFERENCES}

1. Abi-Jaoudeh N, Kruecker J, Kadoury S, Kobeiter H, Venkatesan AM, Levy E, et al. Multimodality image fusion guided procedures: technique, accuracy and applications. Cardiovasc Intervent Radiol 2012;35:986-98.

2. Klein AJ, Tomkowiak MT, Vigen KK, Hacker TA, Speidel MA, Vanlysel MS, et al. Multimodality image fusion to guide peripheral artery chronic total arterial occlusion recanalization in a swine carotid artery occlusion model: unblinding the interventionalist. Catheter Cardiovasc Interv 2012;80:1090-8.

3. Glöckler M, Halbfaß J, Koch A, Achenbach S, Dittrich S. Multimodality 3D-road-map for cardiovascular interventions in congenital heart disease e a single-center, retrospective analysis of 78 cases. Catheter Cardiovasc Interv 2013;82:436-42.

4. Kobeiter H, Nahum J, Becquemin JP. Zero-contrast thoracic endovascular aortic repair using image fusion. Circulation 2011;124:e280-2.

5. Dijkstra ML, Eagleton MJ, Greenberg RK, Mastracci T, Hernandez A. Intraoperative C-arm cone-beam computed tomography in fenestrated/branched aortic endografting. J Vasc Surg 2011;53:583-90.

6. Tacher V, Lin M, Desgranges P, Deux JF, Grünhagen T, Becquemin JP, et al. Image guidance for endovascular repair of complex aortic aneurysms: comparison of two-dimensional and three-dimensional angiography and image fusion. J Vasc Interv Radiol 2013;24:1698-706.

7. Lindholt JS. Radiocontrast induced nephropathy. Eur J Vasc Endovasc Surg 2003;25:296-304.

8. Haddad F, Greenberg RK, Walker E, Nally J, O'Neill S, Kolin G, et al. Fenestrated endovascular grafting: the renal side of the story. J Vasc Surg 2005;41:181-90.

9. Maurel B, Sobocinski J, Perini P, Guillou M, Midulla M, Azzaoui R, et al. Evaluation of radiation during EVAR performed on a mobile c-arm. Eur J Vasc Endovasc Surg 2012;43:16-21.

10. Fukuda T, Matsuda H, Doi S, Sugiyama M, Morita Y, Yamada M, et al. Evaluation of automated 2D-3D image overlay system utilizing subtraction of bone marrow image for EVAR: feasibility study. Eur J Vasc Endovasc Surg 2013;46:75-81. 



\section{CHAPTER}

\section{Quantification of respiratory movement of the aorta and side branches}

A.M. Sailer

B.A. Wagemans

M. Das

P.J. Nelemans

M.W. de Haan

J.E. Wildberger

G.W.H. Schurink

Journal of Endovascular Therapy 2015 Dec;22(6):905-11 


\section{Abstract}

Objective: To assess and quantify the magnitude and direction of respiratory movement of the aorta and origins of its side branches.

Methods: A quantitative 3-dimensional (3D) subtraction analysis of computed tomography (CT) scans during inspiration and expiration was performed to determine the respiratory geometric movements of the aorta and side branches in 60 patients. During breath-hold expiration and inspiration, 1-mm-thick CT slices of the aorta were acquired in unenhanced and contrast-enhanced scans. The datasets were compared using dedicated multiplanar reformation image subtraction software to determine the change in position of relevant anatomic sections, including the ascending thoracicaorta (AA), the origins of the brachiocephalic artery (BA) and left subclavian artery (LSA), the descending thoracic aorta (DTA) at the level of the tenth thoracic vertebra, as well as the origins of the celiac trunk, superior mesenteric artery, and the renal arteries.

Results: Complex movement was visible during inspiration; the regions of interest in the thoracic aorta and side branches moved in the anterior, medial, and caudal directions compared with the expiration state. Mean 3D movement vectors ( \pm standard deviation) were $8.9 \pm 3.6 \mathrm{~mm}(\mathrm{AA}), 12.0 \pm 4.1 \mathrm{~mm}(\mathrm{BA}), 11.1 \pm 3.9 \mathrm{~mm}(\mathrm{LSA})$, and $4.9 \pm 2.5 \mathrm{~mm}$ (DTA). Abdominal side branches moved in the caudal direction $1.3 \pm 1.1 \mathrm{~mm}$. There was significantly less movement in the DTA compared to AA $(p<0.001)$. The correlation coefficient between the extent of LSA movement and thoracic excursion was 0.78 .

Conclusion: The aorta and side branches undergo considerable respiratory movement. The results from this study provide an important contribution to understanding aortic dynamics. 


\subsection{INTRODUCTION}

Advantages in perioperative survival have made endovascular aortic repair the first line treatment for anatomically suitable aneurysms and complicated dissections, ${ }^{1-3}$ while new device developments have expanded the application of endovascular repair to complex thoracoabdominal aneurysms and aneurysms in the arch and ascending aorta. Fusion image guidance was developed as a means to reduce iodinated contrast volume and radiation dose during these complex endovascular repairs. ${ }^{4}$ Despite proven benefits, overlay inaccuracies between the planning computed tomography angiography (CTA) and live fluoroscopy still impair its application. One of the main reasons for these inaccuracies is the displacement of the vessels due to respiratory movement. ${ }^{5}$ These respiratory aortic movements may also influence performance of fenestrated and branched endografts, as long-term stentgraft durability can be hampered by endoleaks, migration, and stent fractures. $^{6-8}$

Former studies have highlighted the influence of myocardial contraction and aging on the aortic spatial position and geometry. ${ }^{9-12}$ Up to now, 3 studies have investigated respiratory vessel movements. The first study was performed on healthy young volunteers using magnetic resonance angiography (MRA), which is limited by motion artifacts. ${ }^{13}$ The other 2 studies using CTA evaluated movements of the peripheral segments of the abdominal side branches ${ }^{14}$ and the aortic arch, ${ }^{15}$ respectively. For comprehensive understanding, it is desirable to gain further knowledge of respiratory movement for the entire aorta and its side branches. Thus, the aim of this study was to assess and quantify the magnitude and direction of 3-dimensional (3D) respiratory movement of the aorta and origins of its thoracic and abdominal side branches in a vascular patient population.

\subsection{MATERIALS AND METHODS}

This was a prospective study approved by the institutional review board (METC-134-40; written informed consent waived) involving 60 consecutive patients (mean age $70 \pm 7$ years; 42 men) referred to the Department of Radiology for diagnostic CTA of the thoracoabdominal aorta. Indications for the biphasic scan protocols were assessment of aortic dissection, postoperative assessment of endovascular aortic repair, and treatment planning in aortic aneurysm patients with prior aortic repair. ${ }^{16}$ Patients were eligible as long as they were able to understand and perform instructed inspiration and expiration maneuvers and were capable of holding their breath for the duration of the scans. Patient characteristics were retrieved from the medical records, and their influence on aortic movement was evaluated in a subgroup analysis. The distribution of patient characteristics is shown in Table 1. 
Table 1. Patient characteristics.

Patient Characteristics

Sex

Male

Female

Mean age \pm SD (range) in years

Mean Body Mass Index \pm SD (range) in $\mathrm{kg} / \mathrm{cm}^{2}$

COPD

Aortic aneurysm

Infra/Juxtarenal

Thoracic

Thoracoabdominal

Aortic dissection

Postoperative after Bentall procedure

TEVAR / EVAR

Stent graft $<5 \mathrm{~cm}$ distally of LSA

Stent graft $>5 \mathrm{~cm}$ distally of LSA

\section{$\mathbf{N}$}

42

18

$$
\begin{aligned}
& 70 \pm 7(42-84) \\
& 26 \pm 4(15-34)
\end{aligned}
$$

16

27

36

60

$8 \quad 13$

16

27

12

20

$4 \quad 7$

$8 \quad 13$

$29 \quad 48$

$12 \quad 20$

$17 \quad 28$

\section{Scan Acquisition}

All scans were performed on a second-generation dualsource CT scanner (Somatom Definition Flash; Siemens Healthcare, Forchheim, Germany). Scanning parameters for the contrast-enhanced scanning phase were $64 \times 0.6-\mathrm{mm}$ slice collimation, $330-\mathrm{ms}$ gantry rotation time, $120-\mathrm{kV}$ tube voltage, and $130-\mathrm{mAs}$ tube current time using $120 \mathrm{~mL}$ of iodinated contrast (Ultravist $300 \mathrm{mg} \mathrm{I/mL;} \mathrm{Berlex} \mathrm{Laboratories,} \mathrm{Wayne,} \mathrm{NJ,} \mathrm{USA)} \mathrm{injected}$ at $6 \mathrm{~mL} / \mathrm{s}$ (iodine delivery rate $1.8 \mathrm{~g} \mathrm{I} / \mathrm{s}$ ). For the non-contrast-enhanced scanning phase, the parameters were $128 \times 0.6-\mathrm{mm}$ slice collimation, 500 -ms gantry rotation time, and $120 \mathrm{kV} / 130 \mathrm{mAs}$. All images were reconstructed at 1-mm slices with an increment of 0.8 $\mathrm{mm}$ using a standard soft tissue kernel (Siemens B 30f). Patients were provided with clear instructions for the breathing maneuver. The unenhanced phase was acquired during breath hold at the level of functional residual capacity, which is the volume of air present in the lungs at the end of passive expiration. For the contrast-enhanced phase, patients were asked to perform maximum inspiration and hold their breath for the duration of the scan. 
Image Analysis

The datasets were processed on a state-of-the-art 3D image workstation (IMPAX, Version 6.5.2; AGFA Healthcare, Mortsel, Belgium). The expiration and inspiration datasets of each patient were automatically matched by means of dedicated multiplanar reformation image subtraction software (IMPAX Volume Viewing 3D). Dataset overlay was checked in all 3 dimensions and adjusted manually when necessary using the spine and table as reference points. Vessel movement analysis was performed semiautomatically by drawing circular regions of interest (ROIs) at predefined anatomic levels of the aorta and origins of the side branches on the inspiratory and expiratory datasets (Figure 1).
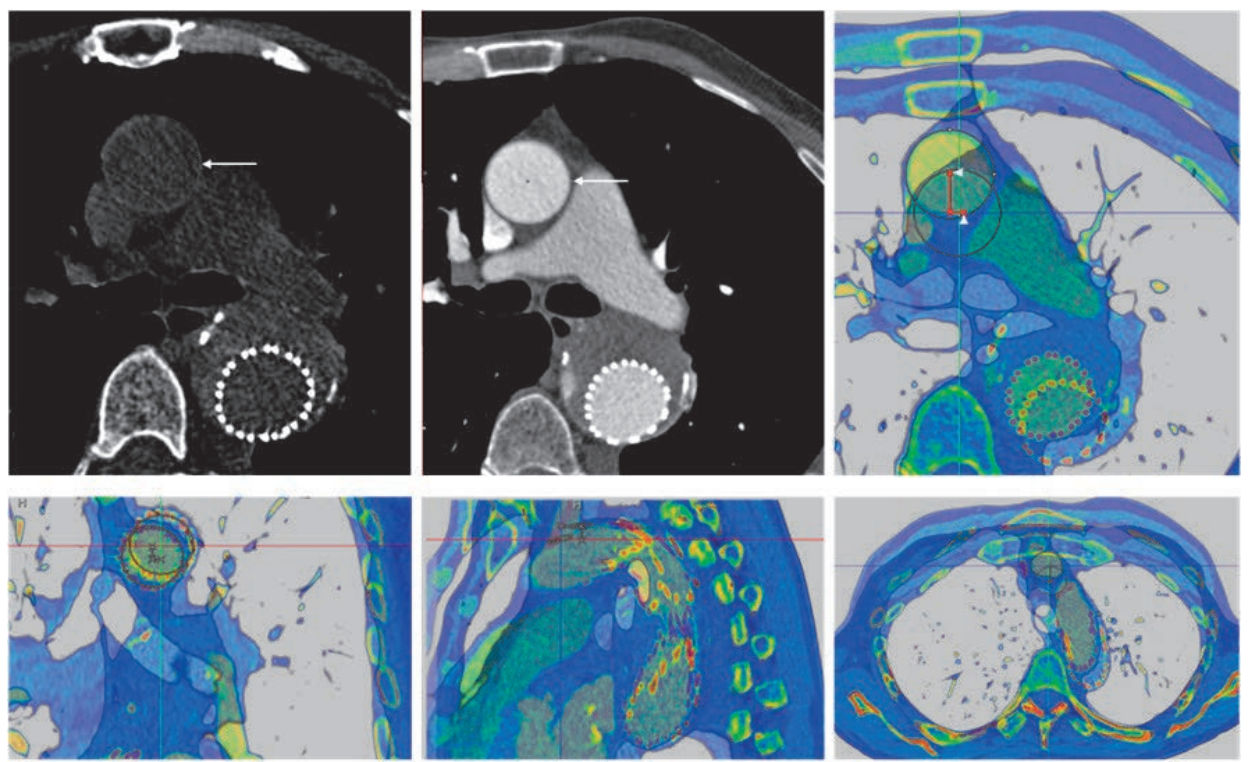

Figure 1. Subtraction analysis of inspiratory and expiratory CT datasets. Upper row: ROls (arrows) drawn manually on predefined aortic levels in expiratory (left image) and inspiratory (middle image) datasets. In-plane geometric differences between the centers of the ROls (arrow heads) calculated in the matched dataset (right image). Lower row: Matched datasets of inspiratory and expiratory scans shown in three dimensions.

These levels included the ascending aorta at the level of the carina; the origins of the brachiocephalic artery, left common carotid artery, and left subclavian artery (LSA); the descending aorta at level of the tenth thoracic vertebra; and the origins of the celiac trunk, superior mesenteric artery, left and right renal arteries, as well as upper poles of the kidneys. The 3D software automatically subtracted and measured the in-plane geometric changes between the ROls on both datasets in axial, coronal, and sagittal views from which 3D displacement vectors were calculated by the law of Pythagoras as introduced elsewhere. ${ }^{10}$ The diaphragm as well as the anterior-posterior (AP) thorax wall position were used as reference for the respiratory phases. The diaphragm dome position 
was measured on both sides in the mid-clavicular line on coronal reconstruction. The APthoracic diameters were measured in the hemithoracic midline on axial planes.

\section{Statistical Analysis}

Quantitative values are presented by means \pm standard deviation. Differences in aortic displacement between the various segments of the aorta were tested for significance using a t test for paired samples. To evaluate the effect of various patient characteristics on aortic movement at the origin of the LSA, mean differences between patient groups were tested for significance using the t test for independent samples. Dependence of vessel movement on thorax excursion was analyzed with linear regression; outcomes are given as the slope, 95\% confidence interval $(\mathrm{Cl})$, and correlation coefficient $\left(r^{2}\right)$. A 2-sided $p<0.05$ was considered significant. Analyses were performed using SPSS statistical software (version 20.0; IBM Corporation, Somers, NY, USA).

\section{$3.3 \quad$ RESULTS}

All patients followed the breathing instructions, and the scans were successfully acquired. With inspiration, the ROls of the thoracic aorta and side branches moved in the anterior, medial, and caudal directions compared with the expiration state; the abdominal side branches moved in the caudal direction.

Table 2. Overview of respiratory movement of various anatomic levels.

Displacement (mean \pm standard devaition and range, values in $\mathbf{m m}$ )

\begin{tabular}{lcccc}
\cline { 2 - 5 } Anatomic level & Anterior-Posterior & Left-Right & Cranio-Caudal & Total Vector \\
\hline Thorax excursion & $9.7 \pm 5.8(0-24)$ & 0 & $28.2 \pm 15.1(4-57)$ & $32.6 \pm 15.1(4.5-60.5)$ \\
AA & $7.0 \pm 3.3(0-15)$ & $5.2 \pm 2.5(0-11)$ & $\mathrm{a}$ & $8.9 \pm 3.3(0-15)^{\mathrm{b}}$ \\
BA & $8.6 \pm 3.2(1-15)$ & $4.1 \pm 1.8(0-8)$ & $7.4 \pm 3.2(0-14)$ & $12.0 \pm 4.1(1-19)$ \\
LCC & $7.4 \pm 2.7(1-13)$ & $3.8 \pm 1.8(0-9)$ & $6.1 \pm 2.7(1-13)$ & $11.1 \pm 3.9(1-19)$ \\
LSA & $7.4 \pm 2.7(1-14)$ & $3.8 \pm 1.8(0-10)$ & $6.2 \pm 2.6(0-12)$ & $11.1 \pm 3.9(2-18)$ \\
DA & $3.3 \pm 1.9(0-8)$ & $3.4 \pm 2.0(1-8)$ & $\mathrm{a}$ & $4.9 \pm 2.5(0-8)$ \\
CT & 0 & 0 & $1.1 \pm 1.1(0-4)$ & $1.1 \pm 1.1(0-4)$ \\
SMA & 0 & 0 & $1.3 \pm 1.2(0-4)$ & $1.3 \pm 1.2(0-4)$ \\
LR & 0 & 0 & $1.5 \pm 1.2(0-5)$ & $1.5 \pm 1.2(0-5)$ \\
RR & 0 & 0 & $1.5 \pm 1.3(0-5)$ & $1.5 \pm 1.3(0-5)$ \\
LKUP & & & $19.6 \pm 13.3(2-55)$ & \\
RKUP & & & $20.6 \pm 14.2(2-54)$ &
\end{tabular}

AA: Ascending aorta at level of the carina. BA: Brachiocephalic artery origin. LCC: Left common carotid artery origin. LSA: Left subclavian artery origin. DA: Descending aorta at level of the carina. CT: Celiac trunk origin. SMA: Superior mesenteric artery origin. LR: Left renal artery origin. RR: Right renal artery origin. LKUP: Left kidney upper pole. RKUP: Right kidney upper pole. a $\mathrm{Z}$-axis movement not adequately measureable. b $2 \mathrm{D}$ vector. 
Respiratory movement was the largest in the ascending aorta and origins of the arch side branches. The quantitative data of ROI movement at various anatomical levels are shown in Table 2. There was significantly less movement in the descending compared to the ascending thoracic aorta $(p<0.001)$. The magnitude of thorax excursion had a significant effect on the LSA origin movement (slope $=0.20,95 \% \mathrm{Cl}$ : 0.16 to $0.25, r^{2}=0.78, p=0.001$; Figure 2). Patients who had a thoracic stent-graft inserted within $5 \mathrm{~cm}$ distal to the LSA showed significantly less vessel movement $(p=0.01)$, while other patient characteristics did not have significant influence on the magnitude of vessel movement (Table 3).

Table 3. Influence of patient characteristics on aortic movement. Mean 3D vectors and standard deviation (in millimeter) presented for the level of the origin of the left subclavian artery. Absolute numbers in brackets.

Patient characteristics

Male (42) vs. Female (18)

COPD yes (16) vs. no (44)

Age $\geq 65$ (49) vs. $<65$ years (11)

BMI $\geq 25$ (34) vs. $<25 \mathrm{~kg} / \mathrm{cm}^{2}(26)$

Stent graft yes (29) vs. No (31)

Stent graft $<5 \mathrm{~cm}$ distally to LSA (12) vs. $\geq 5 \mathrm{~cm}$ distally to LSA or no stent graft (48)

Bentall yes (8) vs. no (52)

Aneurysm yes (36) vs. no (24)

Dissection yes (4) vs. no (56)

$\begin{array}{ccc}\text { Mean } \pm \text { SD } & \text { Mean } \pm \text { SD } & \text { P - value } \\ 11.0 \pm 4.3 & 11.4 \pm 2.8 & .09 \\ 10.8 \pm 4.2 & 11.7 \pm 3.3 & .34 \\ 11.0 \pm 4.0 & 11.6 \pm 3.9 & .76 \\ 11.6 \pm 4.4 & 9.9 \pm 3.1 & .14 \\ 10.7 \pm 4.5 & 11.2 \pm 3.4 & .19 \\ 10.2 \pm 2.1 & 11.4 \pm 4.5 & .01 \\ 9.9 \pm 4.4 & 11.2 \pm 3.9 & .65 \\ 10.4 \pm 4.2 & 12.1 \pm 3.5 & .22 \\ 11.0 \pm 4.0 & 11.8 \pm 2.7 & .39\end{array}$

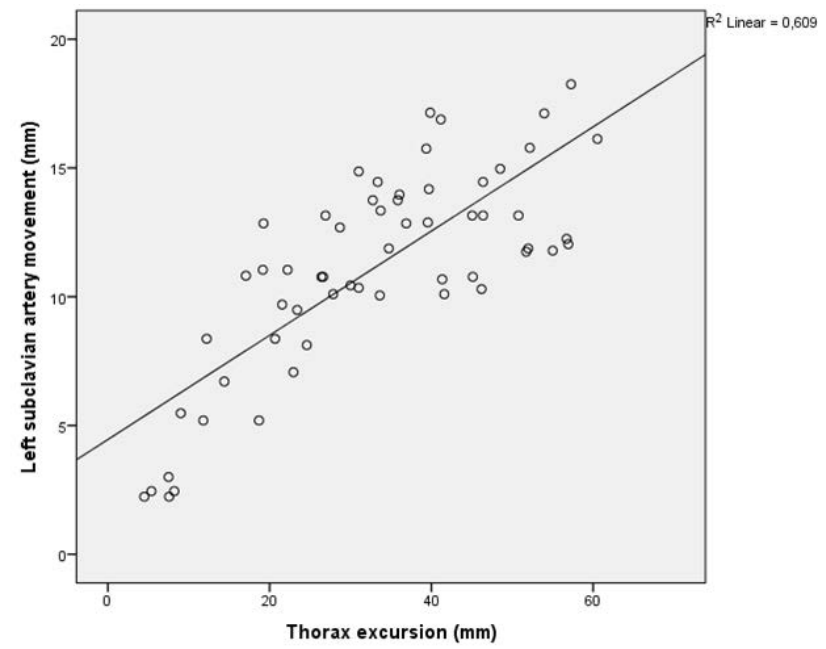

Figure 2. Dependence of 3D left subclavian artery origin movement on thorax excursion. Entire population shown in this figure. 


\subsection{DISCUSSION}

This study has shown that the magnitude of respiratory movement is largest in the ascending aorta and arch, gradually decreasing along the descending aorta to the abdominal side branches. These findings need to be discussed in the context of both branched and fenestrated repair of the aortic arch and ascending aorta and the accuracy of fusion image guidance during stent-graft implantation.

When interpreting the results of this study, it is important to note that the observed magnitudes of movement represent the differences between forced inspiration and expiration in a vascular patient population. Most of the patients followed the breathing instructions intensely; 5 showed $<1 \mathrm{~cm}$ thoracic excursion between inspiration and expiration (Figure 2). In these patients, compound 3D LSA movement was $<4 \mathrm{~mm}$. With tighter respiration, the aortic movements strongly correlated with the magnitude of thorax excursion, with an average 2-mm LSA movement for every centimeter of thoracic excursion. The presented values in Table 2 therefore depend on the patients' individual ability to take a deep breath.

Respiratory aortic movement is expected to be larger with larger thoracic excursions. This finding is corroborated by the results of the study from Weber et al., ${ }^{13}$ who found ascending aorta movements during respiration as large as $24.3 \pm 6.0$ millimeters and descending aorta movements of $18.2 \pm 5.5$ millimeters in a young healthy population. Although these magnitudes may not be reached during tidal breathing in the majority of vascular patients or in ventilation during general anesthesia, it is important for surgeons to be aware of respiratory aortic movements for precise deployment of stent grafts near supra-aortic branches, as the range of movements is not visible on the pre-deployment aortogram acquired during ventilatory arrest. We therefore recommend deploying thoracic stent grafts during identical ventilatory arrest status as during angiography.

We assessed the influence of various patient characteristics on the magnitude of aortic movement. Differences between most patient subgroups were small and nonsignificant, but the results should be carefully interpreted considering the relatively small sample size in some of the groups. We found that endograft insertion in the proximal descending aorta stabilizes the geometry, resulting in significantly less movement during respiration. In the study population, there were no patients included with stent-grafts in the arch or ascending aorta. However, with the demonstrated greater respiratory movement in this segment, it seems plausible that higher physical forces from respiratory movement will expose the stent-grafts to significantly greater mechanical stress in this region compared to the descending and abdominal aorta. The influence of physical forces on the durability of stent-grafts will have to be evaluated in further studies; however, some conjectures can be made based on the results of this study. 
The abdominal aorta was seen to be relatively stable during respiration, and only minimal cranio-caudal movement was registered in the origins of the renal and visceral branch origins. This is consistent with the known good durability of renal and visceral branches from branched and fenestrated thoracoabdominal aneurysm repairs. Incidences of branch stent fractures, migration, and branch-related rupture are low during long-term follow-up. ${ }^{17}$

The aortic arch and ascending aorta are the last frontiers in endovascular aortic repair, but data on the durability of these stent-grafts are not yet available. Regarding the demonstrated respiratory forces in this region, stent-grafts will have to absorb these movements in order to prevent material fatigue. The main movements in the supra-aortic branch vessel origins were in the AP as well as cranio-caudal directions. The quantitative results of the study might provide input for stent-graft design, as chosen materials and structures will have to cope with the mechanical stress implied from respiratory movement. From a theoretical standpoint, higher flexibility of the graft components might be needed for a robust and durable repair in the proximal aorta. In order to limit complications such as stent fracture, component disconnection, and migration and to enhance durability, a branched stent-graft design with self-expanding stents seems favorable compared to a fenestrated graft design with balloon-expandable stents.

The ascending aorta is exposed to physiologic strain from cardiac movement. ${ }^{10,11}$ As our aortic scan protocol did not include electrocardiogram-gated or triggered scanning, we could not account for the magnitude of heart beat- related vessel movements, which is a limitation of the study. Cranio-caudal movements in the ascending aorta are expected to be influenced by heart movement. ${ }^{18,19}$ To overcome misinterpretation, we therefore do not present data for cranio-caudal movement of the ascending aorta as we are not able to distinguish between vessel movement caused by respiration vs heart beatinduced movement in this region.

A further limitation of the scan protocol lies in the comparison of an inspiratory contrastenhanced to an expiratory unenhanced phase. A small pilot study carried out in advance showed that contrast injection during the expiratory phase was not necessary as the aortic border was sufficiently visible on the noncontrast phase. Theoretically uneven data quality between the contrast-enhanced and unenhanced phases may have hampered accuracy of the ROls drawn on the unenhanced phase, thereby influencing the correctness of the center of the ROI and the quantitative subtraction analysis. This would lead to so-called nondifferential misclassification and may have masked even larger movements.

Concerning fusion guidance, previous studies have pointed out inaccuracies of the overlay roadmap. ${ }^{5,20}$ Preprocedure diagnostic CTA scans are commonly acquired during breath hold inspiration to prevent motion artifacts during scanning, which results in overlay mismatch due to the different respiratory state during diaphragm relaxation 
under general anesthesia at endovascular surgery. Fully relying on fusion guidance for fenestrated stent-graft positioning and deployment at the level of the aortic arch is hazardous and could result in accidental malposition and side branch coverage due to respiration-related inaccuracies of the fusion roadmap at this level.

For fusion guidance during prosthesis placement in standard and complex endovascular aneurysm repair, it is crucial to know the range of expected inaccuracies of the origins of the renal arteries and other abdominal side branches. We found that average respiratory movement of the abdominal side branches does not exceed $1.5 \pm 1.3 \mathrm{~mm}$ (range 0 - 5); movement is furthermore present only in the caudal direction during inspiration. This is in line with the data from Fukuda et al, ${ }^{20}$ who reported an average $2 \pm 2$ - $\mathrm{mm}$ (range 0 7) fusion image mismatch during EVAR at the level of the renal arteries in the craniocaudal direction. One possibility to reduce these fusion image overlay inaccuracies would be to perform diagnostic CTAs during slight instead of forced inspiration or during expiration.

In conclusion, computed tomography angiography subtraction technology provides an important contribution to understanding aortic dynamics. The magnitude of respiratory aortic movement is the largest in the ascending aorta and arch, gradually decreasing along the descending aorta to the abdominal side branches. The results from this analysis may have implications for design and implantation of complex aortic stentgrafts, especially when involving the aortic arch and its side branches.

\section{REFERENCES}

1. Greenberg RK, Lu Q, Roselli EE, et al. Contemporary analysis of descending thoracic and thoracoabdominal aneurysm repair: a comparison of endovascular and open techniques. Circulation. 2008;118:808-817.

2. Walsh SR, Tang TY, Sadat U, et al. Endovascular stenting versus open surgery for thoracic aortic disease: systematic review and meta-analysis of perioperative results. J Vasc Surg. 2008;47:1094-1098.

3. Nienaber CA, Kische S, Rousseau H, et al; INSTEAD-XL trial. Endovascular repair of type B aortic dissection: longterm results of the randomized investigation of stent grafts in aortic dissection trial. Circ Cardiovasc Interv. 2013;6: 407-416.

4. Maurel B, Hertault A, Sobocinski J, et al. Techniques to reduce radiation and contrast volume during EVAR. J Cardiovasc Surg (Torino). 2014;55(2 suppl 1):123-131.

5. Sailer AM, de Haan MW, Peppelenbosch AG, et al. CTA with fluoroscopy image fusion guidance in endovascular complex aortic aneurysm repair. Eur J Vasc Endovasc Surg. 2014;47:349-356.

6. Demers P, Miller DC, Mitchell RS, et al. Midterm results of endovascular repair of descending thoracic aortic aneurysms with first-generation stent grafts. J Thorac Cardiovasc Surg. 2004;127:664-673.

7. Rits J, van Herwaarden JA, Jahrome AK, et al. The incidence of arterial stent fractures with exclusion of coronary, aortic, and non-arterial settings. Eur J Vasc Endovasc Surg. 2008;36:339-345. 
8. Tanaka A, Takahashi S, Saito S. Migration of fractured renal artery stent. Catheter Cardiovasc Interv. 2011;77:305-307.

9. van Prehn J, Vincken KL, Muhs BE, et al. Toward endografting of the ascending aorta: insight into dynamics using dynamic cine-CTA. J Endovasc Ther. 2007;14:551-560.

10. Weber TF, Ganten MK, Böckler D, et al. Heartbeat-related displacement of the thoracic aorta in patients with chronic aortic dissection type B: quantification by dynamic CTA. Eur J Radiol. 2009;72:483-488.

11. Rengier F, Weber TF, Henninger V, et al. Heartbeat-related distension and displacement of the thoracic aorta in healthy volunteers. Eur J Radiol. 2012;81:158-164.

12. Fleischmann D, Hastie TJ, Dannegger FC, et al. Quantitative determination of age-related geometric changes in the normal abdominal aorta. J Vasc Surg. 2001;33:97-105.

13. Weber TF, Tetzlaff R, Rengier F, et al. Respiratory displacement of the thoracic aorta: physiological phenomenon with potential implications for thoracic endovascular repair. Cardiovasc Intervent Radiol. 2009;32:658-665.

14. Suh GY, Choi G, Herfkens RJ, et al. Respiration-induced deformations of the superior mesenteric and renal arteries in patients with abdominal aortic aneurysms. J Vasc Interv Radiol. 2013;24:1035-1042.

15. Suh GY, Beygui RE, Fleischmann D, et al. Aortic arch vessel geometries and deformations in patients with thoracic aortic aneurysms and dissections. J Vasc Interv Radiol. 2014;25:1903-1911.

16. Ueda T, Fleischmann D, Rubin GD, et al. Imaging of the thoracic aorta before and after stent-graft repair of aneurysms and dissections. Semin Thorac Cardiovasc Surg. 2008;20: 348-357.

17. Mastracci TM, Greenberg RK, Eagleton MJ, et al. Durability of branches in branched and fenestrated endografts. J Vasc Surg. 2013;57:926-933.

18. Beller CJ, Labrosse MR, Thubrikar MJ, et al. Finite element modeling of the thoracic aorta: including aortic root motion to evaluate the risk of aortic dissection. J Med Eng Technol. 2008;32:167-170.

19. Kozerke S, Scheidegger MB, Pedersen EM, et al. Heart motion adapted cine phase-contrast flow measurements through the aortic valve. Magn Reson Med. 1999;42: 970-978.

20. Fukuda T, Matsuda H, Doi S, et al. Evaluation of automated 2D-3D image overlay system utilizing subtraction of bone marrow image for EVAR: feasibility study. Eur J Vasc Endovasc Surg. 2013;46:75-81. 



\title{
CHAPTER
}

\section{Radiation exposure of abdominal cone beam computed tomography}

\author{
A.M. Sailer \\ G.W.H. Schurink \\ J.E. Wildberger \\ R. de Graaf \\ W.H. van Zwam \\ M.W. de Haan \\ G.J. Kemerink \\ C.R.L.P.N. Jeukens
}

Cardiovascular and Interventional Radiology

2015 Feb;38(1):112-20 


\section{ABSTRACT}

Objective: To evaluate the patients' radiation exposure of abdominal C-arm cone beam computed tomography (CBCT).

Methods: This prospective study was approved by the institutional review board; written informed consent was waived. Radiation exposure of abdominal CBCT was evaluated in forty patients who underwent $\mathrm{CBCT}$ during endovascular interventions. Dose Area Product (DAP) of CBCT was documented and effective dose (ED) was estimated based on organ doses using dedicated Monte Carlo simulation software with consideration of Xray field location and patients' individual body weight and height. Weight dependent ED per DAP conversion factors were calculated. CBCT radiation dose was compared to radiation dose of procedural fluoroscopy. CBCT dose related risk for cancer was assessed.

Results: Mean ED of abdominal CBCT was $4.3 \mathrm{mSv}$ (95\% Cl: 3.9; $4.8 \mathrm{mSv}$, range 1.1 to 7.4 $m S v)$. ED was significantly higher in the upper than in the lower abdomen $(p=0.003)$ and increased with patients' weight $(r=0.55$, slope $=0.045 \mathrm{mSv} / \mathrm{kg}, \mathrm{p}<0.001)$. Radiation exposure of $\mathrm{CBCT}$ corresponded to the radiation exposure of on average 7.2 fluoroscopy minutes ( $95 \% \mathrm{Cl}: 5.5 ; 8.8$ minutes) in the same region of interest. Lifetime risk of exposure related cancer death was $0.033 \%$ or less depending on age and weight.

Conclusion: Mean effective dose of abdominal CBCT was 4.3 milliSievert depending on Xray field location and body weight. 


\subsection{INTRODUCTION}

C-arm cone beam Computed Tomography (CBCT) is an advanced imaging technology that acquires volumetric "CT-like" images by flat panel detectors in state-of-the-art interventional angiography suites. Multiple viewing planes in three-dimensional datasets and enhanced soft tissue contrast provide substantial improvement to conventional fluoroscopy and angiography. ${ }^{1}$ In recent years, various clinical applications of CBCT have been evaluated for vascular and non-vascular interventional procedures and reports of use emerge in medical literature. These include biopsy and ablation needle guidance ${ }^{2-4}$, endovascular road-mapping and catheter guidance ${ }^{5-9}$, immediate multiplanar posttreatment assessment ${ }^{6,10}$ and soft tissue imaging. ${ }^{11,12}$ These features may enhance procedural efficacy and safety but the body of data on radiation exposure is still limited.

$\mathrm{CBCT}$ should not result in additional radiation exposure for the medical staff, as all members are usually outside the interventional suite during acquisition. For patients, the application of $\mathrm{CBCT}$ may lead to an increase of procedural exposure to radiation, but a net reduction of fluoroscopy time and angiogram series due the availability of better spatial information is also possible. ${ }^{5,13}$ (Non-uniform) exposure to ionizing radiation can be assessed in terms of equivalent organ doses and effective dose (ED), which are expressed in units of Sievert, typically in the milliSievert (mSv) range. The organ doses allow an estimation of the attributable risk to develop lethal malignancy later in life. ${ }^{14,15}$ Aim of our study was to evaluate patients' radiation exposure of abdominal C-arm cone beam computed tomography (CBCT).

\subsection{MATERIALS AND METHODS}

This prospective study was approved by the institutional review board; informed consent was waived. CBCT radiation exposure was prospectively evaluated in patients with thoraco-abdominal aneurysms and other complex vascular diseases who underwent fenestrated or branched endovascular aortic repair (FEVAR / BEVAR) or endovascular recanalization and stenting procedures between June 2012 and August 2013. In all patients, CBCT was performed prior to the intervention for image fusion guidance. Figure 1 shows an example of abdominal CBCT co-registered to multidetector computed tomography (MDCT) angiography.

\section{Acquisitions}

Abdominal CBCT was acquired at our angiography suite through rotational movement of the C-arm covering a 180-degree circular under couch trajectory (Allura Xper FD20, Philips Healthcare, Best, The Netherlands). Volumetric datasets were automatically sent to a 3D 
workstation for multiplanar viewing and image co-registration. All technical parameters of the CBCT acquisition are summarized in Table 1. Standard parameters of fluoroscopic imaging were a total beam filtration of $0.4 \mathrm{~mm}$ copper and $3.5 \mathrm{~mm}$ aluminum, 15 frames per second and automatic exposure control (AEC).

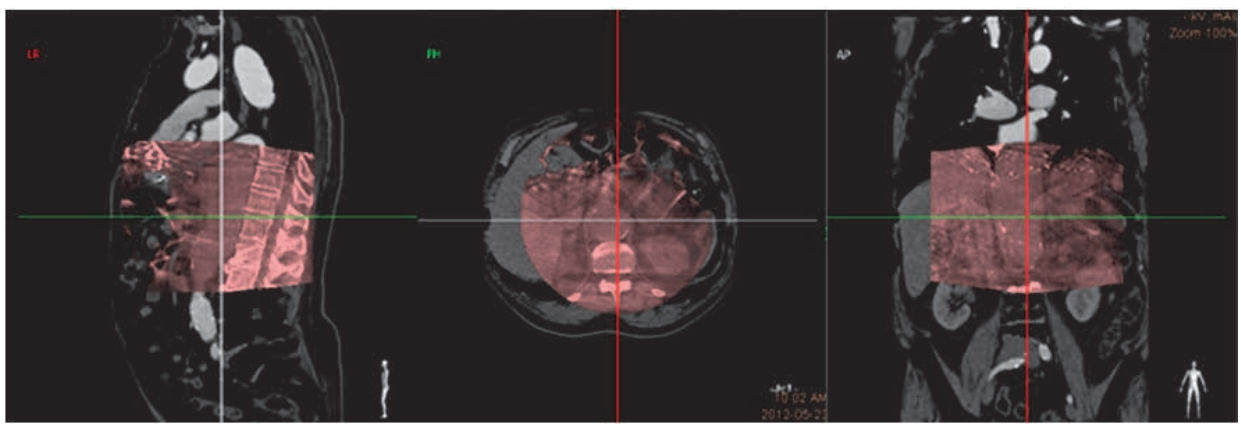

Figure 1. Volumetric CBCT dataset (pink) coregistered to MDCT dataset (grey) in a patient with thoracoabdominal aortic aneurysm. CBCT was acquired in the patient's upper abdomen just before the intervention for image fusion guidance during BEVAR.

Table 1. Technical parameters of abdominal CBCT acquisition with automatic exposure control

\begin{tabular}{|lc|}
\hline Parameter & Abdominal CBCT \\
\hline Name & CT Abdomen LD Roll \\
Tube voltage & $117-123 \mathrm{kV}^{*}$ \\
Tube current & $142-325 \mathrm{~mA}^{*}$ \\
mAs value & $269-1027 \mathrm{mAs} *$ \\
Pulse width & $6-10 \mathrm{~ms}$ \\
Speed & 30 frames / s \\
Exposure / acquisition time & $10.5 \mathrm{~s}$ \\
Angle of image acquistion & $180^{\circ}$ \\
Number of projections & 316 \\
Source - image distance & $120 \mathrm{~cm}$ \\
Focus - isocentre distance & $81 \mathrm{~cm}$ \\
Inherent filtration & $2.5 \mathrm{~mm}$ aluminum equivalent \\
Added filtration & $1 \mathrm{~mm}$ aluminum $+0.9 \mathrm{~mm}$ copper \\
Detector & Dynamic Flat Panel, Trixell, Pixium 4700 \\
Image matrix & $2480 \times 1910$ pixels \\
Pixel pitch & $154 \mu \mathrm{m}$ \\
Detector size & $30 \times 38 \mathrm{~cm}$ \\
Field of view CBCT (height x width x depth) & $19 \mathrm{~cm} \times 25 \mathrm{~cm} \times 25 \mathrm{~cm}$ \\
Reconstructed slice thickness & $0.98 \mathrm{~mm}$ \\
\hline
\end{tabular}

* values depend on body characteristics and vary during acquisition due to automatic exposure control 


\section{Radiation exposure analysis}

For all procedures, radiation exposure of the CBCT run and total procedure in terms of Dose Area Product (DAP; in $\mathrm{Gycm}^{2}$ ) as well as total procedural fluoroscopy time were registered in the fluoroscopy unit's radiation dose report. For the last seventeen procedures, standardized radiation dose structured reporting (RDSR) had become available wherein the DAP values of procedural fluoroscopy were also registered. Patients' individual body weight and height at the date of the intervention were documented. Precise CBCT X-ray field location was displayed on the scout image of each CBCT run. CBCT X-ray field was focused on the upper abdomen in twenty-four patients and on the lower abdomen in sixteen. Figure 2 demonstrates exemplary the X-ray field and included organs for upper and lower abdominal CBCT as drawn on the PCXMC phantom.

\section{Effective dose estimation}

The effective dose (ED) was estimated by means of a dedicated Monte Carlo software program (PCXMC v2.0, STUK, Helsinki, Finland) in which a size adjustable mathematical hermaphrodite phantom model is used (Figure 2). The phantom incorporates 29 different organs and tissues for which organ doses can be calculated under consideration of patient size, exposure geometry and beam quality. With PCXMC software, organ doses were calculated for every patient separately based on CBCT technical parameters (Table 1) and patients' individual DAP values and body characteristics. To this extent, the 180degree rotation was divided into nineteen projections with a ten-degree increment each. For each angle, we ensured that the center of the (hermaphrodite) software phantom remained in the isocenter of rotation, i.e. the focus-skin distance and entrance field size were adjusted according to the thickness of the phantom. The equivalent dose of all organs was estimated by the software for each projection using 1/19 of the total CBCT DAP value. The corresponding ED was calculated by means of ICRP 103 tissue weighting factors. ${ }^{14}$ Total ED of CBCT represents the sum of the nineteen projections. Calculations were separately repeated for each patient with adjustment of patient's individual body weight and height as well as precise CBCT X-ray field location and mean individual kV value. Weight dependent ED per DAP conversion factors and their variation with projection angles were calculated.

\section{Comparison of CBCT with fluoroscopy}

The average fluoroscopy time corresponding to a radiation exposure equivalent to the radiation exposure of the $\mathrm{CBCT}$ was estimated for each patient individually by dividing the CBCT DAP by the fluoroscopy DAP rate. The fluoroscopy DAP rate is calculated as the total fluoroscopy DAP divided by the total fluoroscopy time. 


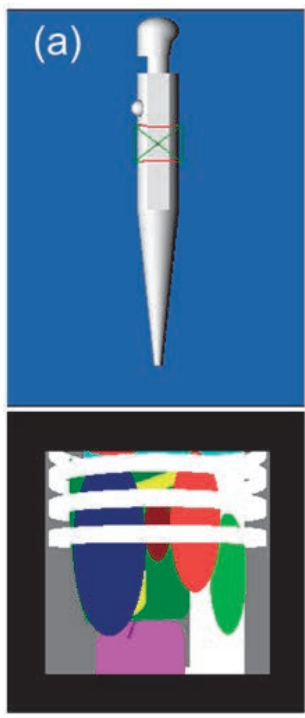

$0^{\circ}=\mathrm{LLAT}$
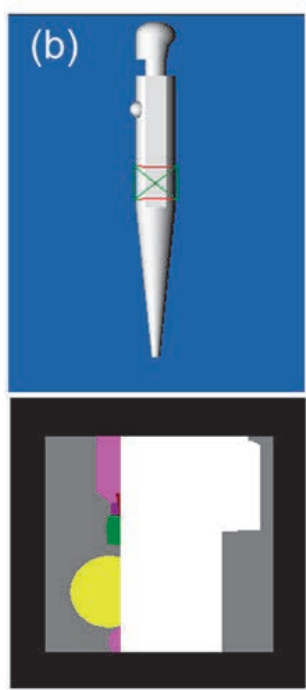

$0^{\circ}=\mathrm{LLAT}$

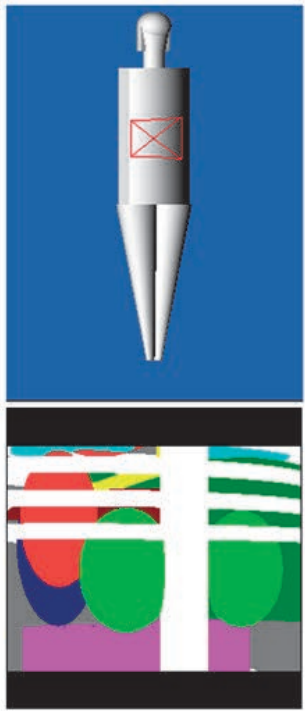

$60^{\circ}$
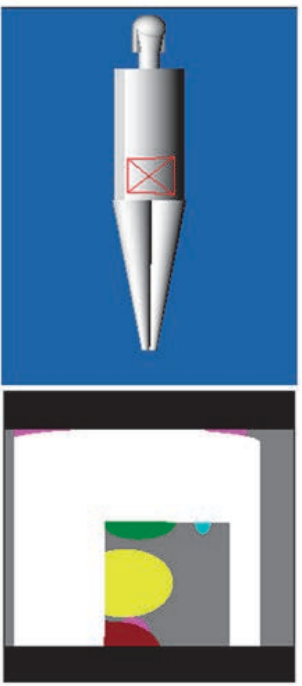

$60^{\circ}$

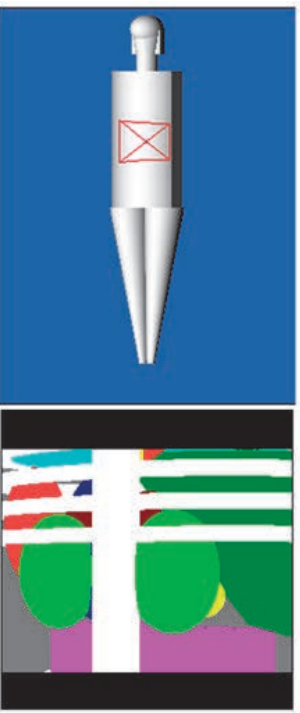

$120^{\circ}$
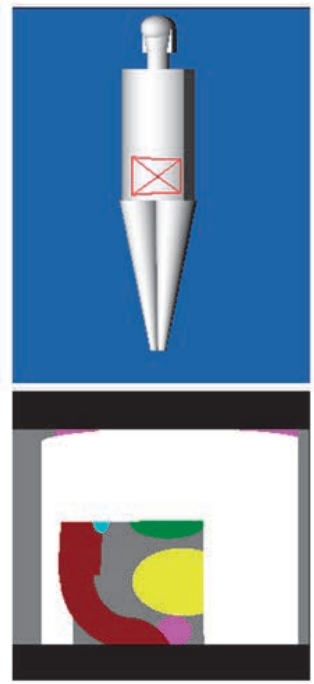

$120^{\circ}$

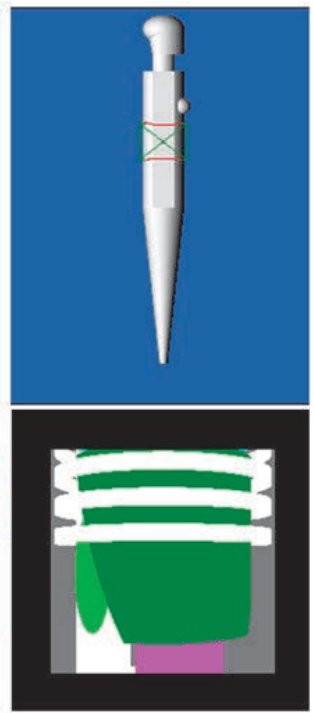

$180^{\circ}=\mathrm{RLAT}$
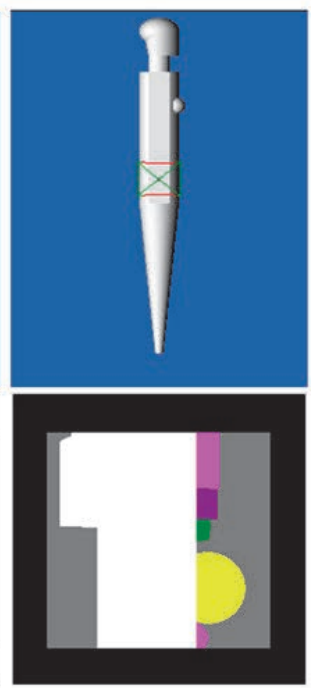

$180^{\circ}=\mathrm{RLAT}$

Figure 2. Upper rows Projection of the X-ray field on the PCXMC phantom exemplary shown for 4 of the 19 steps. Bottom rows schematic representation of the organs present in each projection. Organs included in upper abdominal CBCT X-ray field (a) lung base (light blue), heart (red), stomach (dark blue), distal esophagus (yellow), liver (dark green), spleen (red), pancreas (brown), kidneys and adrenals (light green), colon (pink), small intestine (pink), gallbladder (yellow), and skeleton (white). Organs included in lower abdominal CBCT Xray field (b) colon (brown), small intestine (pink), urinary bladder (yellow), female and male reproductive organs (red, green, and light blue), prostate (pink), and skeleton (white). LLAT left lateral detector position; RLAT right lateral detector position. 


\section{Assessment of exposure related cancer risk}

Assessment of the cancer risk due to $\mathrm{CBCT}$ exposure was performed using the PCXMC software. The risk estimates are based on the organ doses, organ specific risk factors, patient age and risk models established by the Biological Effects of Ionizing Radiation (BEIR) VII committee ${ }^{15}$ resulting in the risk of exposure induced death (REID).

\section{Statistical Analysis}

Differences in radiation exposure and patient characteristics between upper and lower abdominal CBCT were tested on statistical significance using the independent samples ttest (SPSS statistics 20.0, Chicago, Illinois). Correlations between patients' body weight and height and $\mathrm{CBCT}$ radiation exposure and equivalent fluoroscopy time were examined using linear regression. Two-sided $p$-values $<0.05$ were considered significant.

\section{$4.3 \quad$ RESULTS}

The study group consisted of twenty-seven male and thirteen female patients with a mean \pm SD age of $68 \pm 12$ years and mean \pm SD body mass index (BMI) of $26.7 \pm 5.1 \mathrm{~kg} / \mathrm{m}^{2}$. Table 2 gives an overview of the average organ doses for the CBCT run and standard error of the mean as well as associated tissue weighting factors. ${ }^{14}$ The radiation dose was highest in the kidneys, spleen, adrenals, pancreas and liver for upper abdominal CBCT and by ovaries, uterus, colon, small intestine, urinary bladder, and active bone marrow for lower abdominal CBCT. Table 3 provides summarized results of CBCT radiation exposure estimation. ED of the CBCT run was estimated to be in the range of $1.1 \mathrm{mSv}$ to $7.4 \mathrm{mSv}$. Mean ED for upper abdominal CBCT was $4.9 \mathrm{mSv}(95 \% \mathrm{Cl}: 4.3 \mathrm{mSv}, 5.4 \mathrm{mSv})$ and mean ED for lower abdominal CBCT was $3.5 \mathrm{mSv}$ (95\% Cl: $3.0 \mathrm{mSv}, 4.1 \mathrm{mSv}$ ); differences between upper and lower abdominal X-ray field location were statistically significant $(p=$ $0.003)$.

There was a significant positive correlation between DAP value and body weight (Figure $3 a ; r=0.76$, slope $0.238 \mathrm{Gycm}^{2} / \mathrm{kg}, \mathrm{p}<0.001$ ) as well as ED and body weight (Figure $3 \mathrm{~b} ; \mathrm{r}$ $=0.55$, slope $=0.045 \mathrm{mSv} / \mathrm{kg}, \mathrm{p}<0.001$ ). The conversion factor ED per DAP decreased with increasing body weight (Figure 3c; $r=0.99$, slope $=-0.00279 \mathrm{mSv} / \mathrm{Gycm}^{2} \mathrm{~kg}, \mathrm{p}<0.001$ for upper abdominal CBCT and $r=0.99$ slope $=-0.00197 \mathrm{mSv} / \mathrm{Gycm}^{2} \mathrm{~kg}, p<0.001$ for lower abdominal CBCT). Adding patients' body height as an independent variable into the regression models yielded no significant regression coefficients for height ( $p>0.05)$. 
Table 2. Mean equivalent organ doses and standard error of the mean for upper and lower abdominal CBCT and associated tissue weighting factors (IRCP 103) exemplary for mean patient size (170 cm, $78 \mathrm{~kg})$ and associated DAP value (14.7 $\left.\mathrm{Gycm}^{2}\right)$.

\begin{tabular}{|lccc|}
\hline \multicolumn{1}{|c}{ Organ } & $\begin{array}{c}\text { Upper abdominal CBCT } \\
\text { Mean dose } \pm \text { SE }(\mathbf{m G y})\end{array}$ & $\begin{array}{c}\text { Lower abdominal CBCT } \\
\text { Mean dose } \pm \text { SE }(\mathbf{m G y})\end{array}$ & $\begin{array}{c}\text { TWF } \\
\text { IRCP 103 }\end{array}$ \\
\hline Active bone marrow & $5.07 \pm 0.71$ & $8.92 \pm 0.8$ & 0.12 \\
Adrenals & $24.22 \pm 1.56$ & $0.34 \pm 0.12$ & 0.009 \\
Brain & $0 \pm 0$ & $0 \pm 0$ & 0.01 \\
Breasts & $0.64 \pm 0.11$ & $0.03 \pm 0.02$ & 0.12 \\
Colon & $4.68 \pm 1.44$ & $10.4 \pm 1.38$ & 0.12 \\
Esofagus & $4.8 \pm 0.59$ & $0.12 \pm 0.05$ & 0.05 \\
Extrathoracic airways & $0.03 \pm 0.01$ & $0 \pm 0$ & 0.009 \\
Gallbladder & $11.43 \pm 2.16$ & $1.68 \pm 0.28$ & 0.010 \\
Heart & $2.79 \pm 0.39$ & $0.09 \pm 0.04$ & 0.011 \\
Kidneys & $32.79 \pm 1.57$ & $1.38 \pm 0.29$ & 0.012 \\
Liver & $15.14 \pm 2.18$ & $0.59 \pm 0.13$ & 0.05 \\
Lung & $2.8 \pm 0.32$ & $0.07 \pm 0.03$ & 0.12 \\
Lymph nodes & $6.05 \pm 1.12$ & $3.11 \pm 0.36$ & 0.009 \\
Muscle & $3.21 \pm 0.51$ & $4.52 \pm 0.7$ & 0.009 \\
Oral mucosa & $0.02 \pm 0$ & $0 \pm 0$ & 0.009 \\
Ovaries & $1.55 \pm 0.55$ & $17.28 \pm 1.99$ & 0.04 \\
Pancreas & $15.52 \pm 2.14$ & $0.64 \pm 0.17$ & 0.009 \\
Prostate & $0.18 \pm 0.09$ & $8.78 \pm 2.04$ & 0.005 \\
Salivary glands & $0.02 \pm 0.01$ & $0 \pm 0$ & 0.01 \\
Skeleton & $4.14 \pm 0.49$ & $5.15 \pm 0.81$ & 0,00 \\
Skin & $2.27 \pm 0.32$ & $2.65 \pm 0.41$ & 0.01 \\
Small intestine & $6.16 \pm 2.12$ & $10.26 \pm 1.15$ & 0.009 \\
Spleen & $24.94 \pm 2.77$ & $0.56 \pm 0.16$ & 0.009 \\
Stomach & $11.45 \pm 1.99$ & $0.87 \pm 0.17$ & 0.09 \\
Testicles & $0.04 \pm 0.02$ & $1.9 \pm 1.02$ & 0.005 \\
Thymus & $0.55 \pm 0.1$ & $0.02 \pm 0.01$ & \\
Thyroid & $0.07 \pm 0.02$ & $0.05 \pm 0$ \\
Urinary bladder & $0.39 \pm 0.15$ & $14.6 \pm 1.9$ & \\
Uterus & $1.29 \pm 0.48$ & & 0.05 \\
\hline
\end{tabular}

TWF: Tissue weighting factor

Radiation exposure of $\mathrm{CBCT}$ corresponded to the radiation exposure of on average 7.2 minutes ( $95 \% \mathrm{Cl}: 5.5$ minutes, 8.8 minutes) live fluoroscopy in the same region of interest. CBCT dose equivalent fluoroscopy time increased significantly with weight $(r=0.53$, slope $=0.188 \mathrm{~min} / \mathrm{kg}, \mathrm{p}=0.034)$. 
Calculated risk of CBCT exposure induced death based on organ doses for various cancer types is shown in Figure 4 as a function of age and body weight. For example, for a 60year-old female patient with a body weight of 78 kilograms, the stochastic risk for exposure related cancer death was $0.0182 \%$ for upper abdominal CBCT and $0.0147 \%$ for lower abdominal $\mathrm{CBCT}$, respectively. Calculated maximum risk for lifetime exposure induced death was $0.033 \%$.
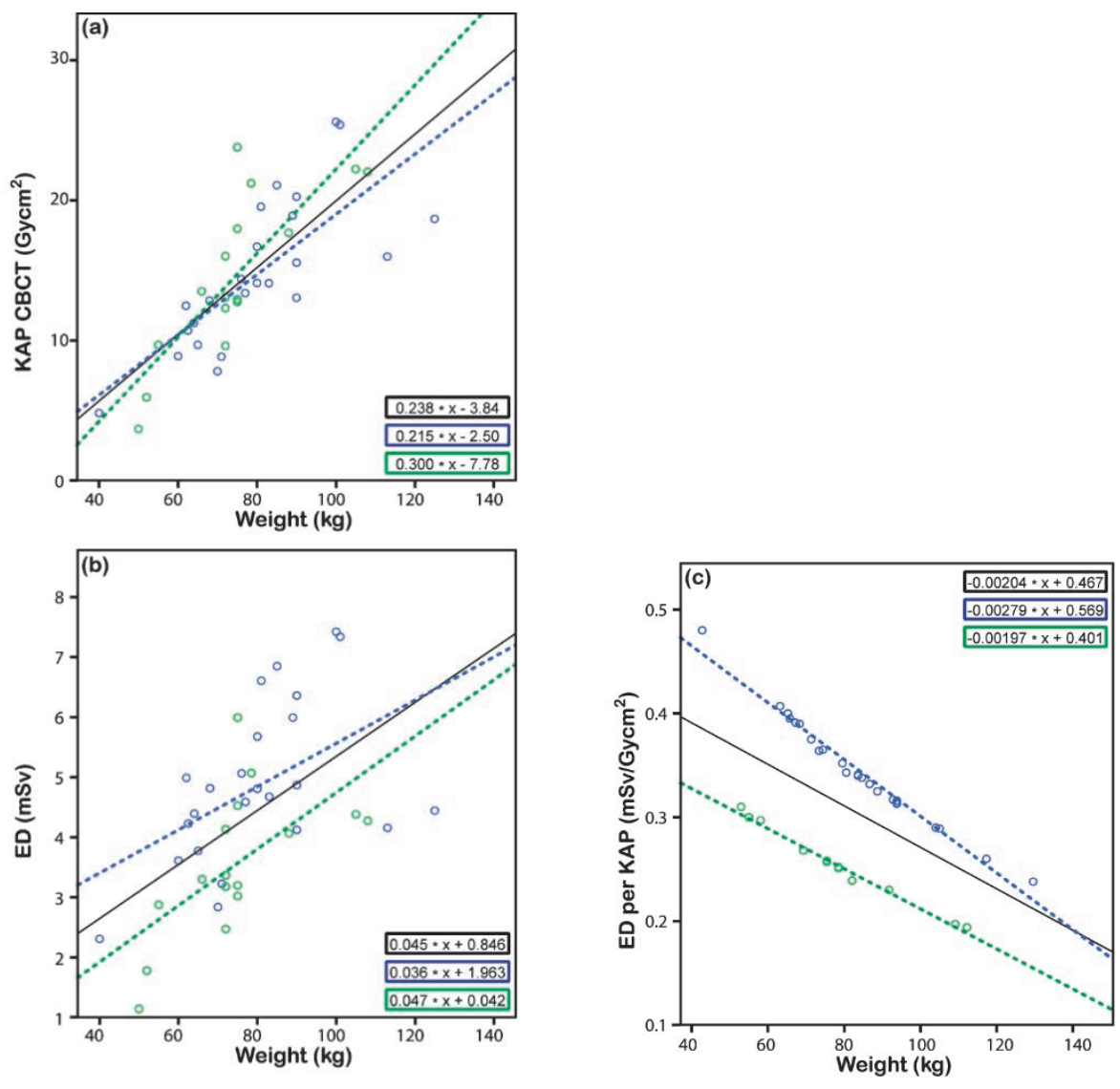

Figure 3. Correlation between a) DAP value versus body weight b) ED versus body weight and c) ED per DAP versus body weight for upper and lower abdominal $C B C T$. Blue symbols and lines upper abdominal $C B C T$. Green symbols and lines lower abdominal CBCT. Black lines all CBCT. 
CHAPTER 4

Table 3. Results of CBCT radiation exposure estimation and impact on total procedure DAP as well as fluoroscopy time equivalence to CBCT.
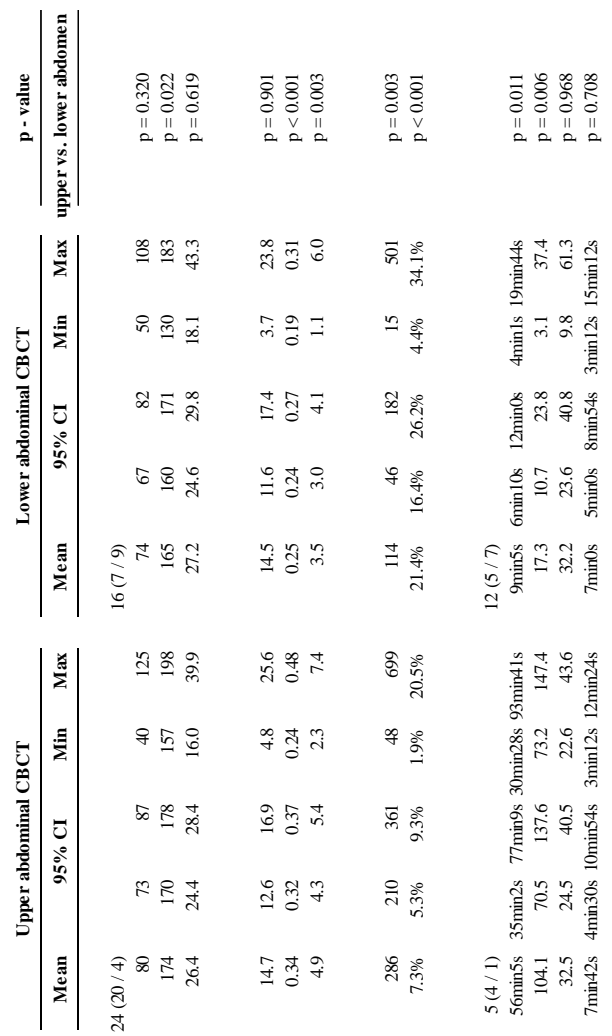

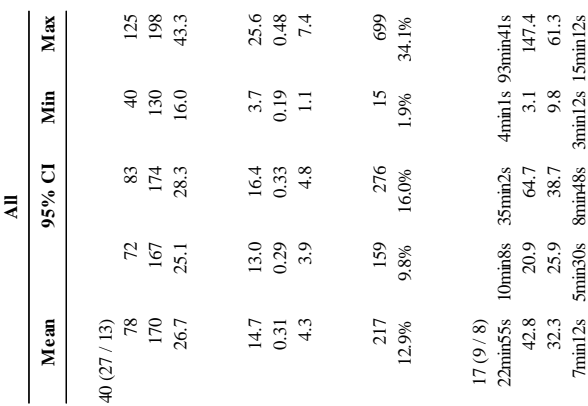

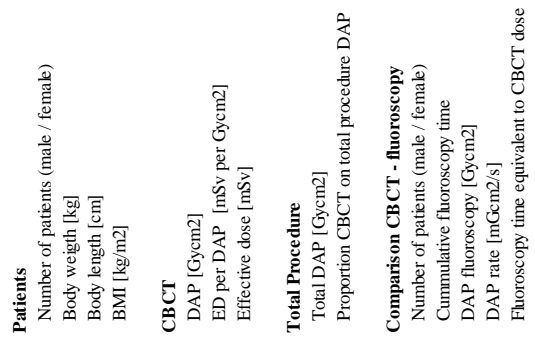



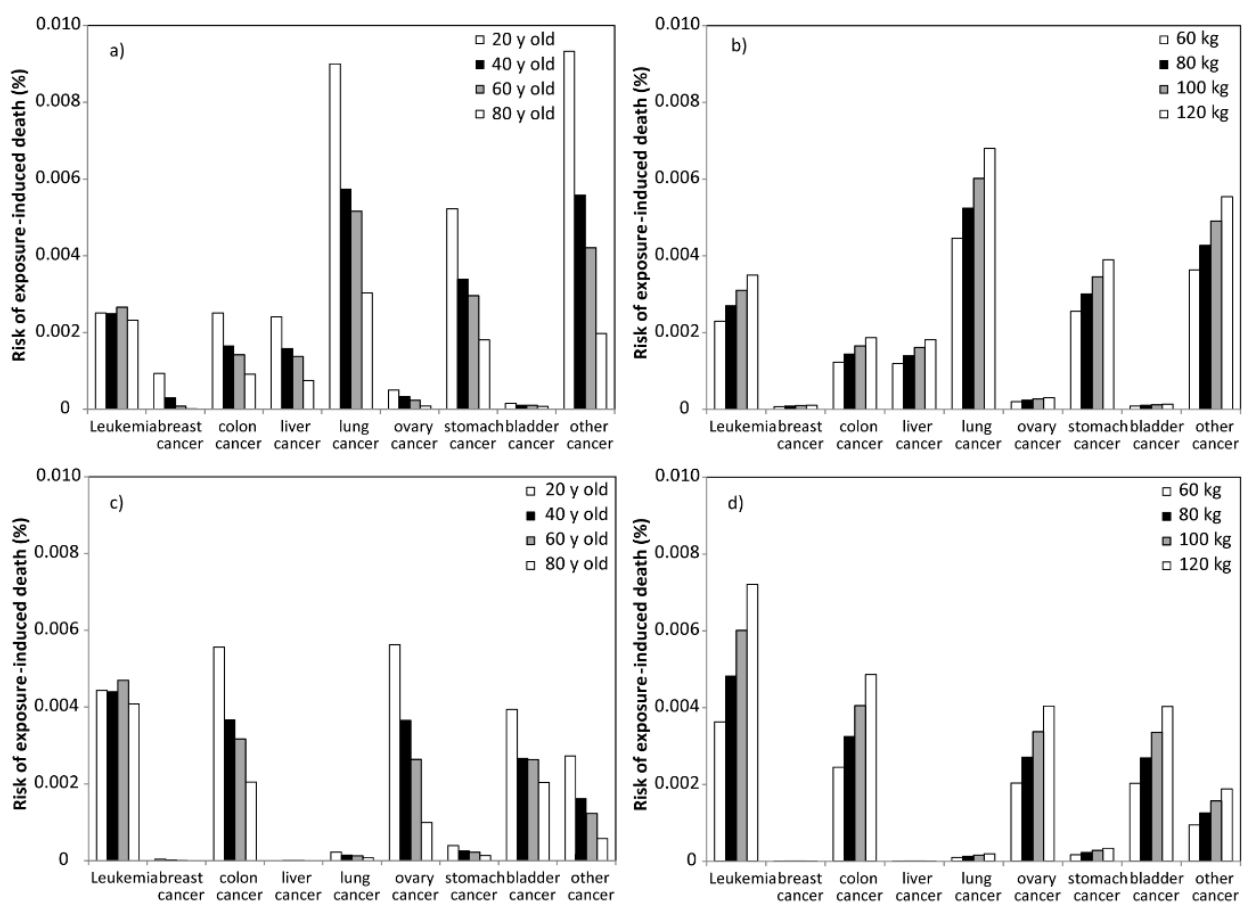

Figure 4. Risk for exposure-induced lethal cancer types for upper abdominal CBCT $(a, b)$ and lower abdominal $\mathrm{CBCT}(\mathrm{c}, \mathrm{d})$ based on individualorgan doses as a function of age $(\mathrm{a}$, $\mathrm{c}$, weight $=80 \mathrm{~kg})$ and weight $(\mathrm{b}, \mathrm{d}$, age $=60$ years).

\subsection{DISCUSSION}

We prospectively evaluated the patient's radiation exposure in forty abdominal CBCT runs by means of dedicated Monte Carlo modelling. Organs positioned in the X-ray field received the highest dose. Average effective dose of CBCT was $4.3 \mathrm{mSv}$, with a range from $1.1 \mathrm{mSv}$ to $7.4 \mathrm{mSv}$. Patient's radiation exposure of abdominal CBCT equals about 1.8 to 2.7 times the annual background radiation dose worldwide and the Netherlands $(2.4 \mathrm{mSv}$ and $1.6 \mathrm{mSv}$ respectively, excluding doses for medical purposes) ${ }^{15,16}$ and about half of the ED of abdominal MDCT ( $8 \mathrm{mSv}$, values reported between 3.5 and $25 \mathrm{mSv}$ ). ${ }^{17,18}$ There was a significant relationship towards lower ED in CBCT with the X-ray field in the lower abdomen compared to upper abdomen $(p=0.003)$ and a strong significant positive correlation between ED and body weight $(p<0.001)$. For correct interpretation of the effective dose values, one must realize the uncertainties present in such estimations. As ED and organ doses cannot be measured in vivo, different approaches are followed. In MDCT, dose estimations are often performed by means of CT dose index (CTDI). Due to the smaller in-plane field of view and larger scan range of CBCT compared to MDCT, the 
CTDI values are not applicable for CBCT though. ${ }^{19,20} \mathrm{~A}$ more accurate method, in line with the definition of ED, is to estimate the organ doses and subsequently calculate the ED using tissue weighting factors. ${ }^{14}$ The organ doses can be measured using an anthropomorphic phantom with integrated thermoluminescent dosemeters (TLDs), or using the DAP-value (displayed by the angiography system) and Monte Carlo software. Main disadvantage of a dosemeter phantom is that the conversion factor ED per DAP does not account for differences in body characteristics - while our data shows that there is a strong weight dependency for the conversion factor. Furthermore, the phantom might not be representative for the actual patient population as illustrated in the TLD phantom study from Tyan et al. evaluating CBCT in liver embolization in the Taiwan population. ${ }^{20}$ They observed much higher DAP values in their patients compared to the phantoms.

To achieve the best possible estimation of the ED of CBCT runs, we performed separate PCXMC Monte Carlo calculations for each patient while precisely accounting for individual CBCT X-ray field location and body characteristics. Moreover, we divided the 180 degree $\mathrm{CBCT}$ run into nineteen projection angles, thereby compensating for the varying phantom thickness by adjusting the focus-skin distance and entrance field size. A limitation is that this method does not incorporate the dose and $\mathrm{kV}$-modulation during the $\mathrm{CBCT}$ rotation by the automatic exposure control as a result of varying body geometry (i.e. the body is thicker in lateral projection compared to PA/AP). To estimate the error introduced, we assessed the angular dependence of DAP and high-voltage during CBCT acquisition by performing separate experiments on an Alderson Rando abdominal phantom while DAPrate and $\mathrm{kV}$ were dynamically recorded on video tape as displayed during rotation. ED was recalculated using the obtained non-uniform DAP and $\mathrm{kV}$ distribution showing that uniformly distributed DAP values and the fixed high-voltage overestimate the ED by $3 \%$.

Prior research has been performed on CBCT radiation exposure. Suzuki et al. performed a TLD phantom study and Monte Carlo simulations based on phantom DAP values to assess the radiation exposure of upper abdominal CBCT in three different angiography units (Siemens Artis dTA, Philips Allura Xper, GE Innova 4100). In the Monte Carlo simulations, they evaluated thirteen projection angles and calculated ED per DAP conversion factors using the former ICRP 60 tissue weighting factors. Doses were estimated for three different phantom sizes. ED of upper abdominal CBCT in a medium size phantom (BMI $23.1 \mathrm{~kg} / \mathrm{m}^{2}$ ) was reported to be in the range from 2.4 to $3.1 \mathrm{mSv}$, depending on the angiography unit's CBCT technical parameters. Largest dose difference between $\mathrm{CBCT}$ protocols from different angiography units was $0.8 \mathrm{mSv} .{ }^{21}$ Analogously to our results, they found a decreasing conversion factor ED per DAP for increasing phantom size. Other studies reported the ED of abdominal CBCT to be in the range from 3.5 to 25.4 $\mathrm{mSv}$, however these results are based on phantom studies or lack detailed information on dose estimation method. $6,12,23-25$ 
Exposure to ionizing radiation introduces risk of developing cancer. The ICRP has established the lifetime detriment adjusted risk coefficient for cancer in the whole population, i.e. comprised of all ages, to be $5.5 \%$ per unit Sievert. ${ }^{14}$ Such a risk estimation based on the ED exhibits inaccuracies as is does not account for age at exposure, current age or body region exposed. We therefore performed accurate risk estimations using the PCXMC software that incorporates organ doses, age, and organ specific risk factors ${ }^{15}$, expressed in lifetime risk of exposure induced cancer death. The risk of overall cancer incidence is about a factor two higher, although this varies for specific cancer types according to their lethality. ${ }^{15}$ The stochastic model for cancer risks below 100 mSv has to be interpreted with caution. The BEIR VII committee reports 95\% confidence intervals for overall cancer mortality and incidence showing that the risks can be estimated within a factor of two. ${ }^{15}$ This means that it is prudent to interpret the lifetime attributable risk (LAR) as an order of magnitude rather than an absolute number. Risk of cancer due to the $\mathrm{CBCT}$ radiation exposure is very low when compared to natural cancer incidence which is $37-46 \%$, and natural risk of lethal cancer which is $18-22 \%$ or one out of five for the US population. ${ }^{15}$ To put CBCT radiation in perspective we performed PXCMC analysis for the whole procedures. In our patient population, mean radiation exposure for FEVAR/ BEVAR procedures was $84.9 \mathrm{mSv}$ (95\% Cl: 62.3; $107.2 \mathrm{mSv}$ ) and $26.5 \mathrm{mSv}$ (95\% Cl: 10.7; $42.2 \mathrm{mSv}$ ) for pelvic procedures. Corresponding risk of cancer therefore was factor 17 and 8 higher respectively compared to the risk from only CBCT exposure. Furthermore, $\mathrm{CBCT}$ radiation has to been seen in the context of the vascular disease management as this patient population is expected to undergo further MDCT during follow-up or may undergo re-interventions which summarizes go considerable lifetime dose.

As any new exposure, $\mathrm{CBCT}$ related radiation has to be discussed in the context of the basic safety principle, i.e. to keep any radiation exposure As Low As Reasonable Achievable (ALARA). CBCT technology might contribute to enhance procedural efficacy and reduce procedural fluoroscopy time. The medical staff is normally in a protected area during $\mathrm{CBCT}$. Therefore, any reduction in fluoroscopy due to the application of CBCT might lead to a welcome reduction of dose incurred by interventional radiologists.

In conclusion, effective dose of abdominal CBCT corresponds to about half of the dose of abdominal multidetector computed tomography (MDCT). Application of CBCT will result in a reduction of patients total procedural radiation exposure if a reduction of fluoroscopy time of approximately seven minutes is achieved. 


\section{REFERENCES}

1. Wallace MJ, Kuo MD, Glaiberman C, Binkert CA, Orth RC, Soulez G. Three-dimensional C-arm cone-beam CT: applications in the interventional suite. J Vasc Interv Radiol. 2008;19(6):799-813.

2. Krücker J, Xu S, Venkatesan A, Locklin JK, Amalou H, Glossop N, Wood BJ. Clinical utility of real-time fusion guidance for biopsy and ablation. J Vasc Interv Radiol. 2011;22(4):515-524.

3. Braak SJ, van Strijen MJ, van Leersum M, van Es HW, van Heesewijk JP. Real-time 3D fluoroscopy guidance during needle interventions: technique, accuracy, and feasibility. AJR Am J Roentgenol. 2010;194(5):W445W451.

4. Meyer BC, Brost A, Kraitchman DL, Gilson WD, Strobel N, Hornegger J, Lewin JS, Wacker FK. Percutaneous punctures with MR imaging guidance: comparison between MR imaging-enhanced fluoroscopic guidance and real-time MR Imaging guidance. Radiology. 2013;266(3):912-919.

5. Sailer AM, de Haan MW, Peppelenbosch AG, Jacobs MJ, Wildberger JE, Schurink GW. CTA with fluoroscopy image fusion guidance in endovascular complex aortic aneurysm repair. Eur J Vasc Endovasc Surg. 2014 doi: 10.1016/j.ejvs.2013.12.022. Epub 2014 Jan 30.

6. Dijkstra ML, Eagleton MJ, Greenberg RK, Mastracci T, Hernandez A. Intraoperative C-arm cone-beam computed tomography in fenestrated/branched aortic endografting. J Vasc Surg. 2011;53(3):583-590.

7. Kobeiter H, Nahum J, Becquemin JP. Zero-contrast thoracic endovascular aortic repair using image fusion. Circulation. 2011;124(11):e280-e282.

8. Deschamps F, Solomon SB, Thornton RH, Rao P, Hakime A, Kuoch V, de Baere T. Computed analysis of threedimensional cone-beam computed tomography angiography for determination of tumor-feeding vessels during chemoembolization of liver tumor: a pilot study. Cardiovasc Interv Radiol. 2010;DOI: 10.1007/s00270-0109846-6.

9. Tam A, Mohamed A, Pfister M, Rohm E, Wallace MJ. C-arm cone beam computed tomographic needle path overlay for fluoroscopic-guided placement of translumbar central venous catheters. Cardiovasc Interv Radiol. 2009;32(4):820-824.

10. Benndorf G, Strother CM, Claus B, Naeini R, Morsi H, Klucznik R, Mawad ME. Angiographic CT in cerebrovascular stenting. AJNR Am J Neuroradiol. 2005;26(7):1813-1818.

11. Möhlenbruch M, Nelles M, Thomas D, Willinek W, Gerstner A, Schild HH, Wilhelm K. Cone-beam computed tomography-guided percutaneous radiologic gastrostomy. Cardiovasc Interv Radiol. 2010;33(2):315-320.

12. Aadland TD, Thielen KR, Kaufmann TJ, Morris JM, Lanzino G, Kallmes DF, Schueler BA, Cloft H. 3D C-arm conebeam CT angiography as an adjunct in the precise anatomic characterization of spinal dural arteriovenous fistulas. AJNR Am J Neuroradiol. 2010;31(3):476-480.

13. Schafer S, Nithiananthan S, Mirota DJ, Uneri A, Stayman JW, Zbijewski W, Schmidgunst C, Kleinszig G, Khanna AJ, Siewerdsena JH. Mobile C-arm cone-beam CT for guidance of spine surgery: image quality, radiation dose, and integration with interventional guidance. Med Phys. 2011;38(8):4563-4574.

14. Valentin J. The 2007 recommendations of the International Commission on Radiological Protection, Annals of the ICRP Publication 103. 2007. Elsevier Science, Oxford.

15. Committee to Assess Health Risks From Exposure to Low Levels of Ionizing Radiation NRC. Health Risks from Exposure to Low Levels of Ionizing Radiation: BEIR VII Phase 2. 2006. National Academic Press, Washington DC. 
16. Rijksinstituut voor Volksgezondheid en Milieu, Ministerie van Volksgezondheid, Welzijn en Sport. Stralingsbelasting in Nederland/Aandeel per stralingsbron. 2013. Available via http://www.rivm.nl/ Onderwerpen/S/Stralingsbelasting_in_Nederland/Aandeel_per_stralingsbron. Accessed 09 Jan 2014.

17. Mettler FA Jr, Huda W, Yoshizumi TT, Mahesh M. Effective doses in radiology and diagnostic nuclear medicine: a catalog. Radiology. 2008;248(1):254-263.

18. van der Molen AJ, Schilham A, Stoop P, Prokop M, Geleijns J. A national survey on radiation dose in CT in The Netherlands. Insights Imaging. 2013;4(3):383-90.

19. Li J, Udayasankar UK, Toth TL, Seamans J, Small WC, Kalra MK. Automatic patient centering for MDCT: effect on radiation dose. AJR Am J Roentgenol. 2007;188(2):547-552.

20. Tyan YS, Li YY, Ku MC, Huang HH, Chen TR. The effective dose assessment of C-arm CT in hepatic arterial embolisation therapy. Br J Radiol. 2013;86(1024):20120551.

21. Suzuki S, Yamaguchi I, Kidouchi T, Yamamoto A, Masumoto T, Ozaki Y. Evaluation of effective dose during abdominal three-dimensional imaging for three flat-panel-detector angiography systems. Cardiovasc Intervent Radiol. 2011;34(2):376-382.

22. Bai M, Liu B, Mu H, Liu X, Jiang Y. The comparison of radiation dose between C-arm flat-detector CT (DynaCT) and multi-slice CT (MSCT): a phantom study. Eur J Radiol. 2012;81(11):3577-3580.

23. Kwok YM, Irani FG, Tay KH, Yang CC, Padre CG, Tan BS. Effective dose estimates for cone beam computed tomography in interventional radiology. Eur Radiol. 2013;23(11):3197-204. doi: 10.1007/s00330-013-2934-7. Epub 2013 Jun 21.

24. Braak SJ, van Strijen MJL, van Es HW, Nievelstein RAJ, van Heesewijk JPM. Effective dose during needle interventions: Cone-Beam CT guidance compared with conventional CT guidance. J Vasc Interv Radiol. 2011;22:455-461.

25. Eide KR, Ødegård A, Myhre HO, Lydersen S, Hatlinghus S, Haraldseth O. DynaCT during EVAR--a comparison with multidetector CT. Eur J Vasc Endovasc Surg. 2009;37(1):23-30. 



\section{CHAPTER}

\section{Occupational radiation exposure during endovascular aortic repair}

A.M. Sailer

G.W.H. Schurink

M.E. Bol

M.W. de Haan

W.H, van Zwam

J.E. Wildberger

C.R.L.P.N. Jeukens

Cardiovascular and Interventional Radiology 2015 Aug;38(4):827-32 


\section{ABSTRACT}

Objective: To evaluate the radiation exposure to operating room personnel and determinants for high personal doses during endovascular aortic repair.

Methods: Occupational radiation exposure was prospectively evaluated during 22 infrarenal aortic repair procedures (EVAR), 11 thoracic procedures (TEVAR) and 11 fenestrated/ branched procedures (FEVAR). Real-time over-lead dosimeters attached to the left breast pocket measured personal doses for the first (FO) and second (SO) operators, radiology technicians (RT), scrub nurses (SN), anesthesiologists (AN) and nonsterile nurses (NSN). Besides protective apron and thyroid collar, no additional radiation shielding was used. Procedural dose area product (DAP), iodinated contrast volume, fluoroscopy time, patients' body weight and C-arm angulation were documented.

Results: Average procedural FO dose was significantly higher during FEVAR $(0.34 \pm 0.28$ $\mathrm{mSv})$ compared to EVAR (0.11 $\pm 0.21 \mathrm{mSv})$ and TEVAR $(0.06 \pm 0.05 \mathrm{mSv} ; \mathrm{p}=0.003)$ Average personnel doses were $0.17 \pm 0.21 \mathrm{mSv}$ (FO), $0.042 \pm 0.045 \mathrm{mSv}$ (SO), $0.019 \pm$ $0.042 \mathrm{mSv}(\mathrm{RT}), 0.017 \pm 0.031 \mathrm{mSv}(\mathrm{SN}), 0.006 \pm 0.007 \mathrm{mSv}$ (AN) and $0.004 \pm 0.009 \mathrm{mSv}$ (NSN). SO and AN doses were strongly correlated with FO dose $(p=0.003$ and $p<0.001)$. There was a significant correlation between FO dose and procedural DAP $(R=0.69, p<$ $0.001)$, iodinated contrast volume $(R=0.67, p<0.001)$ and left-anterior C-arm projections $>60$ degrees $(p=0.02)$ and a weak correlation with fluoroscopy time $(R=$ 0.40, $p=0.049)$.

Conclusion: Average FO dose was a factor four higher than SO dose. Predictors for high personal doses are procedural DAP, iodinated contrast volume, and left-anterior C-arm projections greater than 60 degrees. 


\subsection{INTRODUCTION}

Radiation dose safety during endovascular procedures remains an important area of concern, not only from a patients' point of view but especially for the medical staff. The increasing number of endovascular interventions for aortic pathology, particularly in challenging anatomies, results in increasing radiation exposure to operating room personnel..$^{1-3}$ Endovascular aortic repair procedures are frequently performed at hybrid operating rooms, where also staff members may be present who are not sufficiently educated in radiobiology, radiation safety and dose reduction principles. ${ }^{4,5}$ Characterization of occupational radiation exposure for operators and supporting staff involved in these procedures therefore is of great interest. Aim of our study was to evaluate the radiation exposure to operating room personnel during endovascular aortic repair procedures performed at a hybrid operating room and to assess determinants for high personnel doses.

\subsection{MATERIALS AND METHODS}

This study was approved by the local ethical committee. Informed consent was obtained from all individual participants included in the study; written informed consent was waived.

Radiation exposure to operating room personnel during endovascular aortic repair procedures was prospectively analyzed. Endovascular aortic repair procedures that were consecutively performed at our hybrid operating room (Allura Xper with ClaritylQ technology, Philips Medical Systems, Best, the Netherlands) between September 2013 and January 2014 were included: The study pool consisted of twenty-two infra-renal aortic procedures (EVAR), eleven thoracic aortic repair procedures (TEVAR) and eleven fenestrated or branched aortic repair procedures (FEVAR). For each procedure, the start and end time of the procedure, the dose area product (DAP; in $\mathrm{Gycm}^{2}$ ), amount of iodinated contrast used (in $\mathrm{ml}$ ), fluoroscopy time (in minutes and seconds), angulation of the C-arm (in degrees, grouped left anterior projection $0-30^{\circ}, 30-60^{\circ}, 60-90^{\circ}$ ) and patients' body weight (in $\mathrm{kg}$ ) were documented in a standardized radiology report. X-ray settings were used in low dose mode, and only occasionally switched to medium or high dose when necessary, e.g. during lateral projection in challenging anatomy. Standard parameters of fluoroscopic imaging were total beam filtration of $0.4 \mathrm{~mm}$ copper and 1 mm aluminum, 15 frames per second, pulse width $4 \mathrm{~ms}$ and automatic exposure control (AEC, median tube voltage $107 \mathrm{kV}$ and median tube current $72 \mathrm{~mA}$ ). Standard parameters for the acquisition (DSA) series were additional beam filtration of $0.1 \mathrm{~mm}$ copper and 1 mm aluminum, 3 images per second, tube voltage $80 \mathrm{kV}$ and AEC (median pulse width 60 
ms and median tube current $450 \mathrm{~mA}$ ). Besides protective lead apron and thyroid collar, no additional radiation dose shielding was used.

Personnel doses were measured by means of real-time dosimeters (DoseAware, Philips Medical Systems, Best, the Netherlands) attached to the left breast pocket on the protective apron of the operating room personnel. The dosimeters were calibrated to measure the personal dose equivalent $\mathrm{Hp}(10)$ (Sv), which serves as an estimator for the effective dose. Cumulative doses and dose rates were displayed on a screen for live feedback during the procedures. Doses were registered for the first (FO), and second (SO) operators, the radiology technicians (RT), scrub nurses (SN), anesthesiologists (AN) and non-sterile assisting nurses (NSN). Cumulative doses and dose rates where retrieved from all dosimeters for each procedure by selecting the individual time frame using a dedicated software (DoseManager, Philips Medical System, Best, the Netherlands). Presence and location of the operating room personnel during the procedures were documented. Figure 1 gives a schematic overview of staff location and their pass of motion during the procedures.

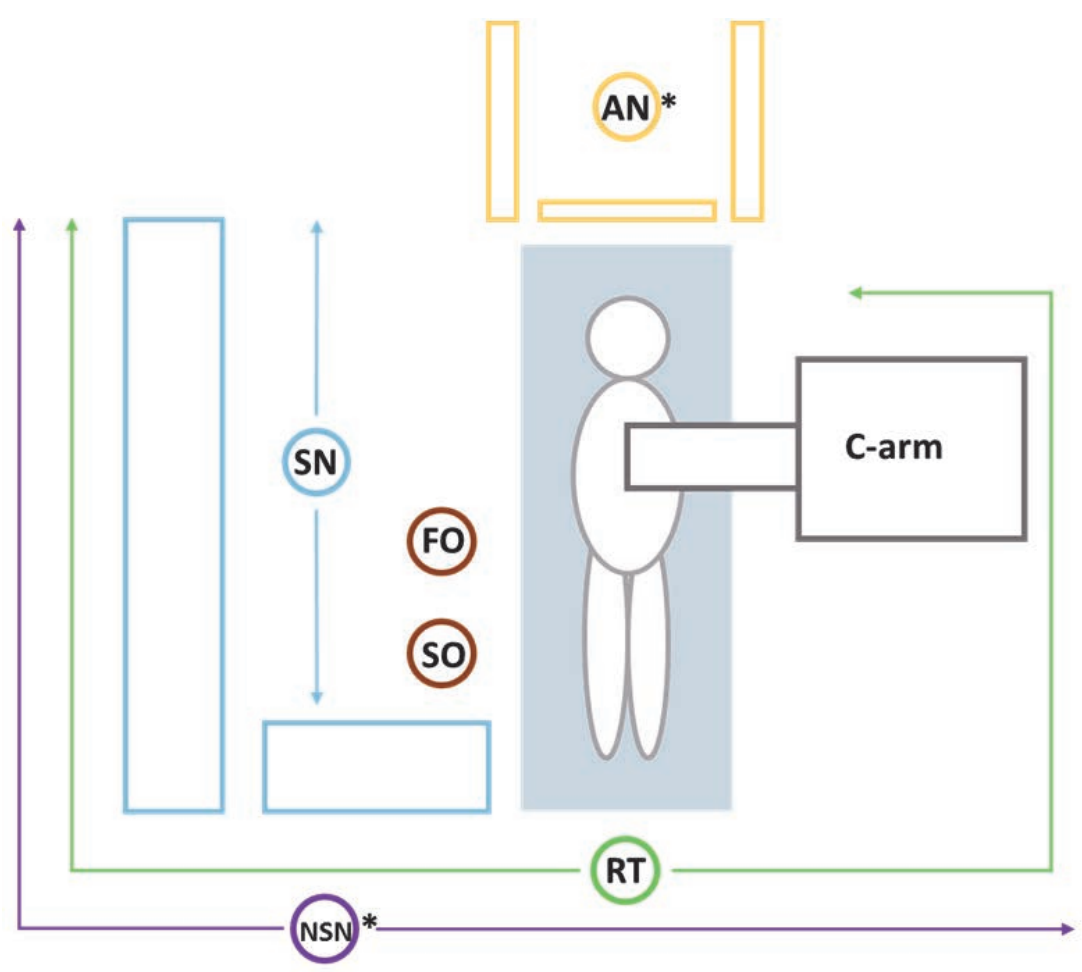

Figure 1. Schematic overview of the position of the medical staff during endovascular aortic repair procedures. FO first operator, SO second operator, SN scrub nurse, AN anesthesiologist, RT radiology technician, NSN nonsterile nurse. Arrow indicating pass of motion. Asterisks indicating operating room personnel occasionally leaving the operation room during the procedure. 


\section{Statistical analysis}

Group differences were tested for statistical significance using the non-parametric Kruskal-Wallis test (SPSS statistics 20.0, Chicago, Illinois). Posthoc analysis was performed using the Mann-Whitney U-test. Correlations between personnel doses and procedure characteristics were examined using linear regression. P-values $<0.05$ were considered significant.

\subsection{RESULTS}

Average procedural occupational over-lead doses $(\mathrm{Hp}(10))$ and procedure characteristics are shown in Table 1. There was a significant correlation between the dose of the FO and procedural DAP $\left(R=0.69\right.$, slope $\left.=1.13 \times 10^{-6} \mathrm{mSv} / \mathrm{Gycm}^{2}, \mathrm{p}<0.001\right)$ and iodinated contrast volume $(R=0.67$, slope $=0.004 \mathrm{mSv} / \mathrm{ml}, p<0.001)$ and a weak correlation with fluoroscopy time $\left(R=0.40\right.$, slope $\left.=1.04 \times 10^{-6} \mathrm{mSv} / \mathrm{min}, \mathrm{p}=0.049\right)$. Usage of left anterior $\mathrm{C}$-arm projections greater than 60 degrees was associated with significantly higher FO doses ( $p=0.019)$. SO and AN doses were significantly correlated with the FO dose $(R=$ 0.69 , slope $=0.131 \mathrm{mSv} / \mathrm{mSv}, \mathrm{p}=0.003$ and $R=0.84$, slope $=0.017 \mathrm{mSv} / \mathrm{mSv}, p<0.001$ respectively).

Group analysis showed that FO doses were significantly different between FEVAR, EVAR and TEVAR procedures $(p=0.003$; Figure 2a). Mean FO dose during FEVAR was $0.34 \pm$ $0.28 \mathrm{mSv}$, mean FO dose during EVAR was $0.11 \pm 0.21 \mathrm{mSv}$ and $0.06 \pm 0.05 \mathrm{mSv}$ during TEVAR. Procedural DAP and fluoroscopy time were different among these groups $(p=$ 0.014 and $p<0.001$ respectively; Figure $2 b$ and $c$ ), while difference in iodinated contrast volume did not reach statistical significance $(p=0.056$; Figure $2 d)$. For EVAR procedures, a significant correlation between FO dose and patient's weight was found $(R=0.561$, slope $=0.003, p=0.024$ ).

\subsection{DISCUSSION}

Radiation exposure during interventional procedures is subject of great interest. While patient radiation exposure is well studied, exposure to operating room personnel did receive less attention in literature so far. This prospective study provides accurate measurements of occupational radiation exposure during abdominal, thoracic and fenestrated endovascular aortic repair procedures performed at a state-of-the art angiosuite. As doses represent over-lead measurements with $0.25 \mathrm{~mm}$ lead-equivalent 
Table 1. Overview of operating room personnel doses and procedure characteristics.

\section{Occupational doses $[\mathrm{mSv}]$}

First operator (FO)

Second operator (SO)

Radiology technician (RT)

Scrub nurse (SN)

Anesthesiologist (AN)

Non-sterile assisting nurse (NSN)
Mean

0.17

0.042

0.019

0.017

0.006

0.004
SD

Min

$\operatorname{Max}$

Procedure characteristics

DAP $\left[\mathrm{Gycm}^{2}\right]$

All

EVAR

TEVAR

FEVAR

Fluoroscopy time [minutes:seconds]

All

EVAR

TEVAR

FEVAR

Iodinated contrast volume [ml]

All

EVAR

TEVAR

FEVAR

Patients' body weight $[\mathrm{kg}]$

All

EVAR

TEVAR

FEVAR

$\begin{array}{rrrr}130 & 131 & 15 & 514 \\ 116 & 122 & 32 & 481 \\ 62 & 46 & 15 & 166 \\ 217 & 159 & 47 & 514\end{array}$

$\begin{array}{rrrr}25: 26 & 19: 47 & 1: 49 & 97: 47 \\ 19: 45 & 8: 21 & 7: 16 & 38: 31 \\ 9: 44 & 7: 20 & 1: 49 & 26: 25 \\ 49: 07 & 21: 50 & 23: 27 & 97: 59\end{array}$

$\begin{array}{llll}92 & 40 & 40 & 200\end{array}$

$\begin{array}{llll}84 & 32 & 50 & 150\end{array}$

$\begin{array}{llll}89 & 49 & 40 & 170\end{array}$

$\begin{array}{llll}116 & 36 & 65 & 200\end{array}$

$\begin{array}{llll}81 & 16 & 47 & 127\end{array}$

$\begin{array}{llll}87 & 18 & 59 & 127\end{array}$

$\begin{array}{llll}73 & 11 & 47 & 85\end{array}$

$\begin{array}{llll}78 & 12 & 57 & 100\end{array}$

No. of procedures Average FO dose

$\begin{array}{ll}17 & 0.06 \mathrm{mSv} \\ 12 & 0.11 \mathrm{mSv} \\ 15 & 0.29 \mathrm{mSv}\end{array}$

SD: Standard deviation. Min: Minimum value. Max: Maximum value. LAO: left anterior oblique.

protective apron and thyroid collar worn by al staff members doses may be reduced by an individual reduction factor depending on the lead-equivalency of the apron and collar as well as radiation beam quality. ${ }^{6,7}$ In our study, doses may be reduced by factor seven to obtain an estimate of the actual body doses of the room personnel. Average doses were highest for the FO with a factor four decrease to the SO who received the second highest dose. This indicates that, besides the increasing distance from the patient, the protective apron and body of the FO work as effective protection shield for 

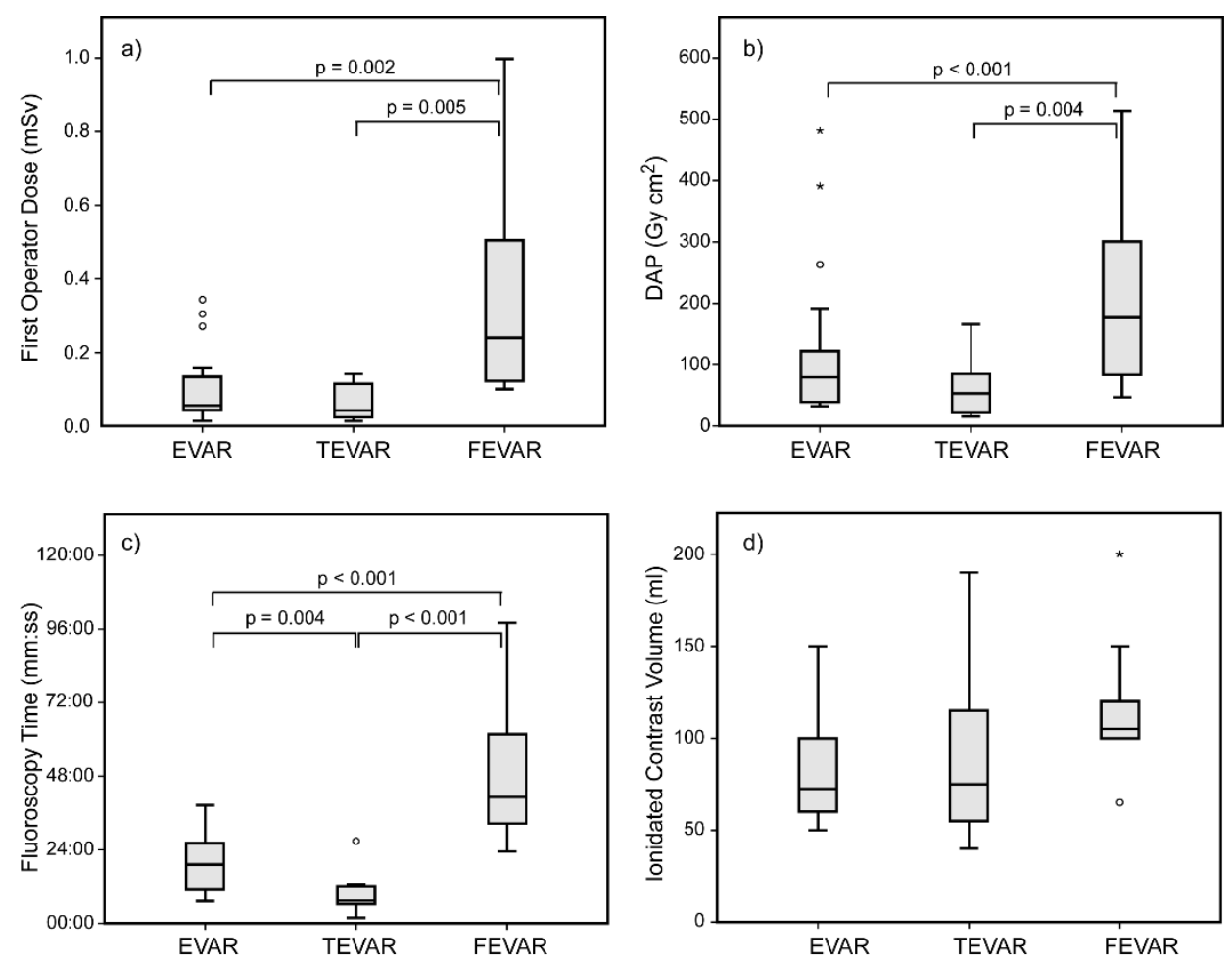

Figure 2. Boxplots of a) first operator doses [Hp(10)], b) DAP values, c) fluoroscopy time, and d) iodinated contrast volume for the EVAR, TEVAR, and FEVAR procedures. Median and interquartile ranges are shown, $p$ values as determined by the post hoc analysis denote statistically significant group differences.

the other medical staff. Highest over-lead dose measured during the study was $1 \mathrm{mSv}$ obtained by the FO during a four-vessel FEVAR procedure. There was a significant correlation between the $\mathrm{FO}$ dose and $\mathrm{SO}$ and $\mathrm{AN}$ doses reflecting the similar pass of motion of these roles during the procedures. Doses of the SN, RT and NSN were not correlated to FO doses indicating that these roles especially may take advantage of their flexible location during the procedure and thereby increasing their distance to the radiation source or standing in the shielding of the operators whenever possible. Average procedural doses were $0.17 \mathrm{mSv}$ for the FO, decreasing to $0.006 \mathrm{mSv}$ and $0.004 \mathrm{mSv}$ obtained by the AN and NSN respectively. The substantially lower doses obtained by the AN and NSN further represent the fact that both roles regularly left the operating room during the procedures.

FO doses obtained during FEVAR were significantly higher compared to EVAR and TEVAR procedures $(p=0.003)$. This is consistent with procedure complexity and the significant positive correlations we found for FO doses and procedural DAP, fluoroscopy time and iodinated contrast volume used. There was a stronger correlation with iodinated contrast volume compared to fluoroscopy time. A potential explanation might be that procedural 
contrast usage serves as an indirect measure for the amount of DSA runs which mainly contributes to the total procedural DAP while fluoroscopy contributes a relatively small fraction for the total radiation dose. ${ }^{8}$ For EVAR procedures, patients' body weight was furthermore significantly correlated to FO dose; group analyses for FEVAR and TEVAR did however not show a strong correlation for body weight, which might be influenced by the limited number of procedures in these subgroups.

During endovascular aortic repair procedures, the medical staff is exposed to two sources of radiation: "leakage radiation" from the X-ray tube and "scatter radiation" originating from the patient. While leakage radiation is regulated by law and only contributes minimally to the obtained dose, scatter radiation is the main contribution to the dose obtained by staff. $^{9}$ The scatter radiation from a point source declines inversely proportional to the square of the distance. This is reflected in the highest doses obtained by the FO and SO, who stand closest to the irradiated body part of the patient. Scatter radiation occurs in all directions; however, backscatter radiation is the highest. ${ }^{9,10}$ During left anterior oblique (LAO) C-arm position the backscatter points in direction of the operator. We indeed saw increasing FO doses with increasing LAO angles. For angles greater than 60 degrees the dose of the first operator was significantly higher compared to lower degrees $(p=0.008)$, emphasizing that these $\mathrm{C}$-arm angulations should be avoided whenever possible.

Recently work has been published on radiation exposure during endovascular aortic repair procedures. ${ }^{11-13}$ Besides differences in fluoroscopy units and acquisition parameters, some general comments can be made. Average FO dose reported by Mohapatra et al. for FEVAR procedures was $0.125 \mathrm{mSv}^{11}$ compared to $0.283 \mathrm{mSv}$ in our study. This difference may be influenced by the fact that they - opposite to our setting applied extra radiation protection measures (two ceiling-mounted transparent shields, table-mounted lead skirts, and a movable "wall shield" for the anesthesiologist), showing the potential value of these additional protection tools in terms of dose reduction. Remarkably the highest average personal dose of $0.27 \mathrm{mSv}$ was measured for the anesthesiologist while we measured only $0.007 \mathrm{mSv}$ for this role, which was further the lowest dose for all staff. This large difference is likely due to the fact that in their study, the dosimeters of the anesthesiologists were attached to an exposed area of the anesthesiology equipment and not to the anesthesiologists' breast pocket (as in our setting), because the anesthesiologists regularly left the operating room. It therefore resulted in an overestimation of their real personal dose. Kloeze et al reported a significant reduction in operator doses during EVAR using disposable radiation-absorbing drapes (Radpad, Worldwide Innovations and Technologies Inc., Kansas City, Missouri) in a non-dedicated angiosuite. ${ }^{12}$ Their FO doses obtained in group with shielding drapes are comparable to the EVAR FO doses from our study on a dedicated fluoroscopy system without additional shielding. In a prospective analysis, Patel el al reported average FO doses of $0.42 \mathrm{mSv}$ and $0.05 \mathrm{mSv}$ for TEVAR and EVAR procedures, respectively. ${ }^{13}$ Average 
FO doses from our study were $0.06 \mathrm{mSv}$ and $0.11 \mathrm{mSv}$ for TEVAR and EVAR. However, in their study, DAP values during TEVAR were much higher than in our study $\left(191 \mathrm{Gycm}^{2}\right.$ versus $62 \mathrm{Gycm}^{2}$ in our study) and were lower during EVAR (97 $\mathrm{Gycm}^{2}$ versus $116 \mathrm{Gycm}^{2}$ in our study).

Stochastic radiation effects from the doses obtained in this study are low, even for highly exposed roles. However, deterministic effects, especially radiation induced posterior subcapsular cataracts, are considerable. ${ }^{14}$ This is reflected by the recent recommendation of the International Commission on Radiological Protection to reduce the equivalent dose limit for eye lenses from 150 to 20 mSv per year. ${ }^{15}$ As distances from irradiated patient's body part to the staff member's breast and eye lenses are comparable, the measured over-lead doses can be taken as approximation of the staff members' procedural eye lens dose. ${ }^{16}$ The ICRP stated that the eye lens dose can be sufficiently assessed using the operational quantity $\mathrm{Hp}(0.07)^{17}$ which can be estimated from the $\mathrm{Hp}(10)$ by means of conversion factors published by the ICRP. ${ }^{18}$ Under the assumption that the radiation exposure (Air Kerma) at the breast level where the dosimeter was positioned in our study is similar to the radiation exposure of the eyes, we estimated that the $\mathrm{Hp}(10)$ doses reported in this paper overestimate the eye lens dose by approximately 0 to $20 \%$. According to this analysis, the first operator would exceed the maximum recommended annual eye lens dose after 58 - 74 FEVAR, 181 - 222 EVAR and 333 - 417 TEVAR procedures, respectively, when not wearing radiation eye protection. However, it should be noted, that this a rough approximation, which may lead to an overestimation of the actual eye lens dose. ${ }^{19,20}$

There are several measures to reduce radiation exposure during endovascular procedures. $8,21,22$ These include well known, generally applicable techniques like optimization of collimation and minimization of fluoroscopy or DSA runs, according to the ALARA principle. In a state of the art hybrid operating room specific additional measures are provided such as flat panel detectors, advanced dose reduction techniques (e.g. Clarity), pulsed fluoroscopy as well as real-time personnel dose monitoring. Large display monitors applied in short distance to the operators allow large size detailed imaging with rare need of magnification. Furthermore, in our clinical practice, we use hybrid image dose reduction measures by fusion of fluoroscopy with preoperative planning CTAs for advanced guidance in complex procedures. ${ }^{23,24}$ Nevertheless, a simple and effective reduction of radiation exposure can be achieved by ceiling-mounted lead glass shields and table-mounted lead skirts as shown by the comparison with the data from Mohapatra et al above; when correctly placed shielding can lead to a reduction of up to $80 \%$ of the staff exposure. ${ }^{25}$ However, these shielding can be unpractical and violate sterility especially when lateral projection is necessary. Also literature shows that Radpad drape is effective for reduction of upper body and hand doses, when not placed in the central beam or obscuring relevant regions. ${ }^{11,26}$ This study and discussion might lead to 
consideration of further optimization of the radiation dose protection measures by the authors and the readers.

In conclusion, the first operator received an average procedural dose of $0.17 \mathrm{mSv}$, which was on average a factor four higher than the second operator who received the second highest average dose. Strong predictors for high personnel doses are procedural dose area product, iodinated contrast volume, and left lateral C-arm angulations greater than 60 degrees.

\section{REFERENCES}

1. Buck DB, van Herwaarden JA, Schermerhorn ML, Moll FL. Endovascular treatment of abdominal aortic aneurysms. Nat Rev Cardiol. 2014 Feb;11(2):112-23.

2. Matsumura JS, Melissano G, Cambria RP, Dake MD, Mehta S, Svensson LG, Moore RD; Zenith TX2 Clinical Trial Investigators. Five-year results of thoracic endovascular aortic repair with the Zenith TX2. J Vasc Surg. 2014 Jul;60(1):1-10.

3. Greenberg RK, Sternbergh WC 3rd, Makaroun M, Ohki T, Chuter T, Bharadwaj P, Saunders A. Fenestrated Investigators. Intermediate results of a United States multicenter trial of fenestrated endograft repair for juxtarenal abdominal aortic aneurysms. J Vasc Surg. 2009 Oct;50(4):730-737.e1.

4. Valentin J. Avoidance of radiation injuries from medical interventional procedures. Ann ICRP. 2000;30(2):767.

5. Kirkwood ML, Arbique GM, Guild JB, Timaran C, Chung J, Anderson JA, Valentine RJ. Surgeon education decreases radiation dose in complex endovascular procedures and improves patient safety. J Vasc Surg. 2013 Sep;58(3):715-21.

6. Eleveld H, Tanzi CP, Ministry of Social Affairs and Employment Netherlands. Standaardisatie van persoonsdosimetrie bij beroepsmatige blootstelling aan ioniserende straling. 2004, Report No. 329.

7. Van Dijk JWE, Ebben L, Franken Y, Netherlands Commission on Radiation Dosimetry Subcommittee Lead Aprons. Code of practice for personal dosimetry of professionals wearing protective clothing during radiological procedures. Report 19 of the Netherlands Commission on Radiation Dosimetry; 2008.

8. Bartal G, Vano E, Paulo G, Miller DL. Management of patient and staff radiation dose in interventional radiology: current concepts. Cardiovasc Intervent Radiol. 2014 Apr;37(2):289-98.

9. Sutton DG, Williams JR. Radiation Shielding for Diagnostic Radiology. The British Institute for Radiology 2012:74-77.

10. Haqqani OP, Agarwal PK, Halin NM, lafrati MD. Defining the radiation "scatter cloud" in the interventional suite. J Vasc Surg. 2013 Nov;58(5):1339-45.

11. Mohapatra A, Greenberg RK, Mastracci TM, Eagleton MJ, Thornsberry B. Radiation exposure to operating room personnel and patients during endovascular procedures. J Vasc Surg. 2013 Sep;58(3):702-9.

12. Kloeze C, Klompenhouwer EG, Brands PJ, van Sambeek MR, Cuypers PW, Teijink JA. Use of disposable radiation-absorbing surgical drapes results in significant dose reduction during EVAR procedures. Eur J Vasc Endovasc Surg. 2014 Mar;47(3):268-72. 
13. Patel AP, Gallacher D, Dourado R, Lyons O, Smith A, Zayed H, Waltham M, Sabharwal T, Bell R, Carrell T, Taylor P, Modarai B. Occupational radiation exposure during endovascular aortic procedures. Eur J Vasc Endovasc Surg. 2013 Oct;46(4):424-30.

14. Vano E, Kleiman NJ, Duran A, Romano-Miller M, Rehani MM. Radiation-associated lens opacities in catheterization personnel: results of a survey and direct assessments. J Vasc Interv Radiol. 2013 Feb;24(2):197204.

15. Broughton J, Cantone MC, Ginjaume M, Shah B. Report of Task Group on the implications of the implementation of the ICRP recommendations for a revised dose limit to the lens of the eye. J Radiol Prot. 2013 Dec;33(4):855-68.

16. van Rooijen BD, de Haan MW, Das M, Arnoldussen CW, de Graaf R, van Zwam WH, Backes WH, Jeukens CR. Efficacy of radiation safety glasses in interventional radiology. Cardiovasc Intervent Radiol 2013 Nov 2. [Epub ahead of print].

17. The 2007 Recommendations of the International Commission on Radiological Protection. ICRP publication 103. Ann ICRP 37:1-33.

18. ICRP (1997) Publication 74: conversion coefficients for use in radiological protection against external radiation. Annals of the ICRP, volume 26/3. Elsevier Science, Oxford.

19. Lie $\varnothing \varnothing$, Paulsen GU, Wøhni T. Assessment of effective dose and dose to the lens of the eye for the interventional cardiologist. Radiat Prot Dosimetry. 2008;132(3):313-8.

20. Sanchez RM, Vano E, Fernandez JM, Ginjaume M, Duch MA. Measurements of eye lens doses in interventional cardiology using osl and electronic dosemeters. Radiat Prot Dosimetry. 2014 Jan 23. [Epub ahead of print].

21. Maurel B, Hertault A, Sobocinski J, Le Roux M, Gonzalez TM, Azzaoui R, Saeed Kilani M, Midulla M, Haulon S. Techniques to reduce radiation and contrast volume during EVAR. J Cardiovasc Surg (Torino). 2014;55(2 Suppl 1):123-31.

22. Scheurig-Muenkler C, Powerski MJ, Mueller JC, Kroencke TJ. Radiation Exposure During Uterine Artery Embolization: Effective Measures to Minimize Dose to the Patient. Cardiovasc Intervent Radiol. 2014 Aug 23. [Epub ahead of print].

23. Sailer AM, de Haan MW, Peppelenbosch AG, Jacobs MJ, Wildberger JE, Schurink GW. CTA with fluoroscopy image fusion guidance in endovascular complex aortic aneurysm repair. Eur J Vasc Endovasc Surg. 2014 Apr;47(4):349-56.

24. Hertault A, Maurel B, Sobocinski J, Martin Gonzalez T, Le Roux M, Azzaoui R, Midulla M, Haulon S. Impact of hybrid rooms with image fusion on radiation exposure during endovascular aortic repair. Eur J Vasc Endovasc Surg. 2014 Jul 17. pii: S1078-5884(14)00310-4.

25. Fetterly KA, Magnuson DJ, Tannahill GM, Hindal MD, Mathew V. Effective use of radiation shields to minimize operator dose during invasive cardiology procedures. JACC Cardiovasc Interv. 2011 Oct;4(10):1133-9.

26. Murphy JC, Darragh K, Walsh SJ, Hanratty CG. Efficacy of the RADPAD protective drape during real world complex percutaneous coronary intervention procedures. Am J Cardiol. 2011 Nov 15;108(10):1408-10 



\title{
CHAPTER
}

Endovascular treatment of complex aortic aneurysms:

Prevalence of acute kidney injury and effect on long-term renal function

\author{
A.M. Sailer \\ P.J. Nelemans \\ C. van Berlo C \\ O. Yazar \\ M.W. de Haan \\ D. Fleischmann \\ G.W.H. Schurink
}

European Radiology 2016 Jun;26(6):1613-9 


\section{ABSTRACT}

Objective: To analyse predictors for short- and long-term renal function changes after fenestrated and branched endovascular aortic repair (EVAR).

Methods: 157 patients underwent fenestrated and branched EVAR. Procedural intraarterial iodinated contrast volume was documented. Serum creatinine and estimated glomerular filtration rate (eGFR) at baseline, during 48 hours following EVAR, at discharge and latest moment of follow-up were recorded. Development of post-EVAR acute kidney injury (AKI; according to AKIN criteria), and potential risk factors for renal failure were recorded. Multivariate regression analyses were used to identify independent risk factors for AKI and eGFR decrease during follow-up.

Results: Forty-three patients (28\%) developed post-EVAR AKI. Long procedure time and occlusion of accessory renal arteries were independent risk factors for development of AKI. (Odds Ratio (OR) 1.005 per minute, 95\% Cl: $1.001-1.01 ; p=0.025$ and OR 3.02, 95\% $\mathrm{Cl}: 1.19-8.16 ; \mathrm{p}=0.029)$. Post-EVAR AKI was associated with a significantly increased risk for eGFR decrease at discharge and latest follow-up (Hazard ratio (HR) 3.47, 95\% Cl: 1.63 - 7.36, $p=0.001$ and $\mathrm{HR} 3.01,95 \% \mathrm{Cl}: 1.56-5.80 ; p=0.001)$. lodinated contrast volume was not an independent risk factor for AKI or eGFR decrease during follow-up.

Conclusion: Development of post-EVAR AKI is an independent risk factor for long-term renal function decrease. 


\subsection{INTRODUCTION}

Advantages in perioperative survival have led endovascular aortic repair (EVAR) to become first line treatment for anatomically suitable aneurysms and complicated acute type B aortic dissections. ${ }^{1,2}$ Developments in aortic stent grafts expanded the field of endovascular therapy towards thoraco-abdominal aneurysms and chronic dissections with insufficient fixation zone. ${ }^{3,4}$ In order to provide adequate graft sealing and, at the same time, preserving aortic side branch patency, the main renal arteries, celiac trunk, superior mesenteric artery and/or supraaortic vessel are catheterized and stented via fenestrations or branches in the aortic main device. Over the last years, fenestrated and branched EVAR procedures have increasingly been performed. Ongoing innovations in devices provide endovascular solutions for more and more challenging anatomy and pathology. The worldwide demographic shift and decreasing indications for open (surgical) aortic repair further contribute to this development. With endovascular procedure complexity, the amount of intra-arterial administered iodinated contrast media increases substantially. At the same time, new techniques like fusion image roadmapping and robotic navigation have been applied and are under further development to reduce procedural iodinated contrast volume and accelerate complex EVAR procedures. ${ }^{5,6}$ For proper risk analysis and adequate procedure management, knowledge on predictors of renal function following complex EVAR is required. Despite numerous research in the field of iodinated contrast, the risk of currently used low-osmolar intraarterial applied iodinated contrast on short-term and long-term renal function decrease remains unclear. ${ }^{7}$ Several studies on renal outcome after endovascular aortic repair have been published, but these studies lack adjustments for contrast volume and other factors by a multivariate risk factor analysis for renal outcome. ${ }^{8-10}$

The aim of this study was to evaluate predictors for short- and long-term renal function changes after complex fenestrated and branched endovascular aortic repair, with special interest in risk factors for $\mathrm{AKI}$ and effect of $\mathrm{AKI}$ on long term-renal damage.

\subsection{MATERIALS AND METHODS}

This retrospective study was approved by the Institutional Review Board; written informed consent was waived. Serum Creatinine ( $\mathrm{SCr}$ ) levels and estimated GFR changes were retrospectively evaluated in 157 consecutive patients who underwent fenestrated and /or branched endovascular aortic repair for juxta-, suprarenal and thoracoabdominal aortic aneurysms between January 2006 and March 2014 at our institution. All procedures were performed under general anaesthesia. Procedural intra-arterial iodinated contrast volume (ialC, low-osmolar, $300 \mathrm{mg}$ iodine / $\mathrm{ml}$ ) and procedure time were documented. SCr levels at baseline, during 48 hours following the intervention, at 
discharge as well as during follow-up were retrieved from the electronic medical patient records. Corresponding estimated glomerular filtration rate (eGFR) levels were calculated using the Modification of Diet in Renal Disease formula. ${ }^{11}$ Patient characteristics, including age, sex, presence of diabetes and congestive heart failure were retrieved from the medical records. Procedure related death was recorded. Procedure related loss of renal parenchyma was assessed by reviewing procedural angiograms together with prepostprocedure CT scans: Angiographically apparent perfusion defects by intentional stent graft coverage of accessory renal arteries as well as iatrogenic renal artery injuries with occlusion of renal artery branches during the EVAR procedures were recorded. Furthermore, it was assessed whether the patients received additional iodinated contrast media during CTA within 48 hours following the EVAR procedure.

\section{Assessment of post-EVAR Acute Kidney Injury (AKI)}

Post-EVAR AKI was defined by comparing the individual post-EVAR SCr measurements to preoperative baseline Scr measurements. According to the definition of the Acute Kidney Injury Network criteria of AKI, patients with (1) an absolute $\mathrm{SCr}$ increase of $\geq 0.3 \mathrm{mg} / \mathrm{dl}$ and / or (2) a relative increase of $\geq 50 \%$ above baseline within 48 hours following the complex EVAR procedures, qualified to have post-EVAR AKI. ${ }^{12}$

\section{Decrease in eGFR during follow-up}

The primary endpoint was decrease in eGFR at discharge. At baseline, discharge and latest moment of follow-up patients were grouped according to their eGFR into five categories: (1) eGFR < $15 \mathrm{ml} / \mathrm{min} / 1.73 \mathrm{~m}^{2}$, (2) eGFR 15 - $29 \mathrm{ml} / \mathrm{min} / 1.73 \mathrm{~m}^{2}$, (3) eGFR 30 $44 \mathrm{ml} / \mathrm{min} / 1.73 \mathrm{~m}^{2}$, (4) eGFR $45-59 \mathrm{ml} / \mathrm{min} / 1.73 \mathrm{~m}^{2}$ and (5) eGFR $\geq 60 \mathrm{ml} / \mathrm{min} / 1.73 \mathrm{~m}^{2}$. Decrease in eGFR was defined as a transition to a category with lower eGFR when compared to baseline. Secondary endpoint was decrease in eGFR at latest moment of follow-up.

\section{Statistical Analysis}

For descriptive purposes, categorical variables were presented as absolute numbers and percentages. Continuous variables were presented as a mean with \pm standard deviations (if normally distributed) or as a median with a range (if not normally distributed). For development of AKI, the effects of risk factors were analysed using multivariate logistic regression. For eGFR decrease at discharge of last follow-up, data were analysed with time-to event analysis to account for differences in follow-up between patients. Multivariable Cox regression analyses were used for calculation of hazard ratios with corresponding 95\% confidence intervals. The hazard ratios represent the independent effect of predefined risk factors on the probability of an event. Observations were 
censored at occurrence of an event (eGFR decrease) or at the end of follow-up. Patients with transient eGFR decrease, with eGFR decrease by one category at discharge but recovery to the eGFR category at baseline, were considered as event-free at the end of follow-up. Predefined risk factors considered relevant as potential predictors were age, sex, baseline eGFR, administered contrast volume, presence or absence of AKI, additional administration of iodinated contrast within 48h post EVAR, renal artery branch occlusion, diabetes and congestive heart failure. P-values $<0.05$ were considered to indicate statistical significance. Analyses were performed with SPSS statistics 20.0, Chicago, Illinois.

\subsection{RESULTS}

The study group consisted of 131 men and 26 women with a mean age of $73 \pm 6$ years. The distribution of baseline characteristics is presented in Table 1 . Median stay until discharge was 6 days (range 2 to 61 days, interquartile range (IQR) 3 - 9) and median time until last follow-up was 380 days (range 16 to 2.762 days, IQR 117 - 925). One patient died perioperatively and this patient was excluded from the analysis because no data were accessible for post-EVAR renal function. Another nine patients died within 30 days (range $3-28$ days) after the intervention due to procedure related complications. Seven of those 9 patients (78\%) developed post-EVAR AKI. Among the other 146 patients, 36 patients (24\%) developed post-EVAR AKI. In total, forty-three patients of 156 patients (28\%) developed post-EVAR AKI. Mean procedural ialC volume was $162 \pm 77 \mathrm{ml}$ (range 50 - $450 \mathrm{ml}$ ). Mean procedural ialC volume in patients who developed post-EVAR AKI was $195 \pm 88 \mathrm{ml}$ (range $50-350 \mathrm{ml}$ ) versus $149 \pm 69 \mathrm{ml}$ (range $65-450 \mathrm{ml}$ ) in patients without post-EVAR AKI $(p=0.001)$. Mean procedure time in patients who developed post-EVAR AKI was $282 \pm 138$ minutes (range $119-603$ minutes) versus $197 \pm 89$ minutes (range 69 - 395 minutes) in patients without post-EVAR AKI ( $p=0.001)$. In twenty-six patients $(17 \%)$, procedure related loss of renal parenchyma was apparent.

The results of the logistic regression analysis evaluating the independent contribution of factors for the development of post-EVAR AKI are shown in Table 2. Development of AKI was significantly associated with procedure time (Odds Ratio (OR) per minute 1.005, 95\% $\mathrm{Cl}: 1.001-1.01, \mathrm{p}=0.001$ ) and renal artery branch occlusion (OR 3.02, 95\% Cl 1.12 $8.16, \mathrm{p}=0.029)$. Although iodinated contrast volume was significantly higher in patients with AKI in the univariate analysis (Table 1), iodinated contrast volume was no longer an independent risk factor for post-EVAR AKI after adjustment for other factors in the multivariate analysis. 
CHAPTER 6

Table 1. Baseline characteristics of the entire patient population $(n=157)$ and distribution between patients with $(n=43)$ and without AKI $(n=113)$.

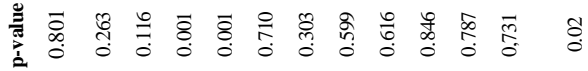

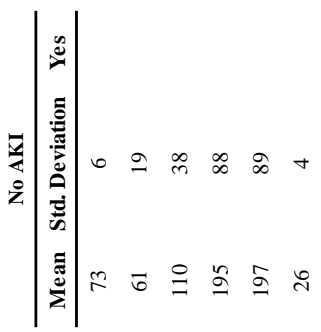

$$
\begin{aligned}
& \text { ळீํำ }
\end{aligned}
$$

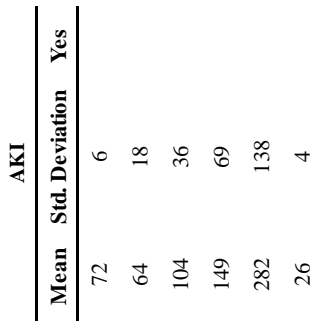

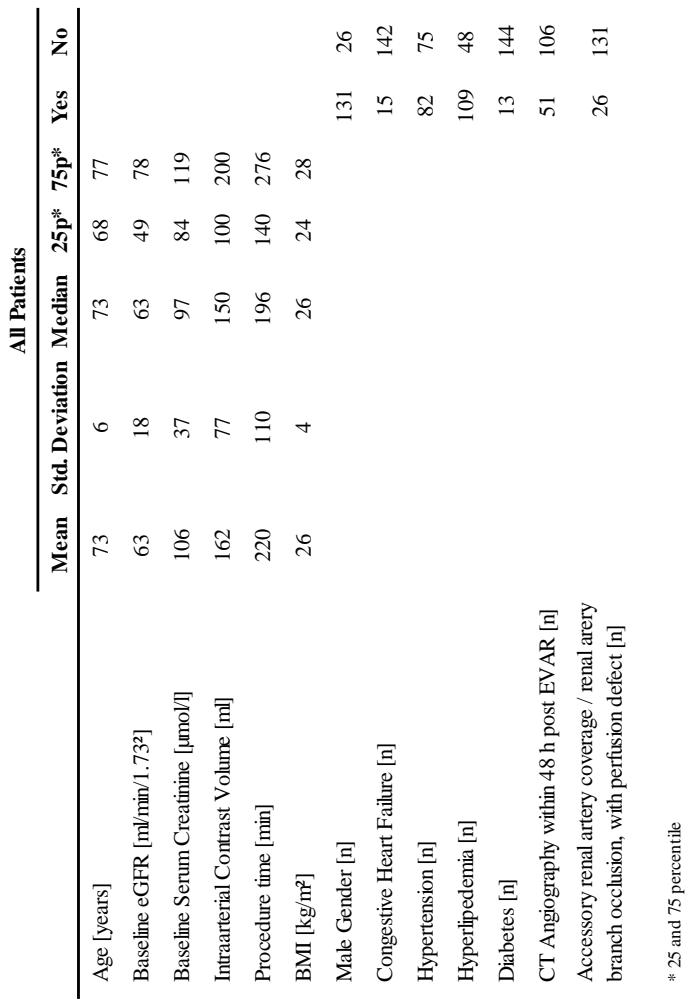


Table 2. Logistic regression analysis for various risk factors for AKI within 48 hours after complex EVAR.

\begin{tabular}{lccc}
\hline & Odds Ratio & $\mathbf{9 5 \%}$ CI & p-value \\
\hline Age (per life-year) & 1.04 & $0.960-1.127$ & 0.334 \\
Sex & 1.357 & $0.415-4.432$ & 0.613 \\
Diabetes & 1.674 & $0.425-6.599$ & 0.426 \\
Congestive heart failure & 1.51 & $0.364-6.270$ & 0.571 \\
Baseline eGFR (per ml/min/1.73m²) & 0.993 & $0.969-1.018$ & 0.605 \\
Iodinated contrast volume (per ml) & 1.003 & $0.997-1.009$ & 0.323 \\
CTA within 48h post EVAR & 1.514 & $0.532-4.310$ & 0.437 \\
Renal artery occlusion & 3.021 & $1.118-8.163$ & 0.029 \\
Procedure time (in minutes) & 1.005 & $1.001-1.010$ & 0.025
\end{tabular}

Table 3 shows the results from the Cox proportional hazard model to assess the independent effects of predefined risk factors on probability of eGFR decrease at discharge. At discharge, the number of patients with eGFR decrease by at least one category was 37. Development of post-EVAR AKI were associated with a significantly increased risk for eGFR decrease at discharge (Hazard Ratio (HR) 3.47, 95\% Cl: 1.63 - 7.36; $p=0.001)$. Non-significantly but slightly increased hazard ratios were observed for accessory renal artery occlusion, iodinated contrast volume, age, mal gender and patients with diabetes $(p>0.05)$.

Table 3. Cox regression analysis for various risk factors for eGFR decrease by at least one category at time of hospital discharge after complex EVAR.

\begin{tabular}{lccc}
\hline & Hazard Ratio & $\mathbf{9 5 \%}$ CI & p-value \\
\hline Age (per lifeyear) & 1.012 & $0.945-1.083$ & 0.74 \\
Sex & 1.169 & $0.423-3.233$ & 0.763 \\
Diabetes & 1.938 & $0.679-5.529$ & 0.216 \\
Congestive heart failure & 0.935 & $0.265-3.306$ & 0.917 \\
Baseline eGFR (per ml/min/1.73m²) & 0.996 & $0.977-1.016$ & 0.722 \\
Iodinated contrast volume (per ml) & 1.002 & $0.996-1.008$ & 0.58 \\
CTA within 48 hours post EVAR & 1.216 & $0.506-2.219$ & 0.662 \\
Renal artery occlusion & 1.97 & $0.866-4.481$ & 0.106 \\
Acute Kidney Injury & 3.467 & $1.633-7.360$ & 0.001 \\
Procedure time (per minute) & 0.997 & $0.993-1.002$ & 0.204
\end{tabular}

Table 4 shows the results for long-term eGFR decrease. At the moment of last follow-up, the number of patients with eGFR decrease by at least one category was 51. Patients with post-EVAR AKI had a significantly increased probability of eGFR decrease at last follow-up 
(HR 3.01, 95\% Cl: $1.56-5.80, p=0.001$ ). Procedure time, iodinated contrast volume and other patient characteristics were not significantly associated with long-term eGFR decrease.

Table 4. Cox regression analysis for various risk factors for eGFR decrease by at least one category at latest moment of follow-up after complex EVAR.

\begin{tabular}{lccc}
\hline & Hazard Ratio & $\mathbf{9 5 \%}$ CI & p-value \\
\hline Age (per lifeyear) & 1.02 & $0.964-1.079$ & 0.5 \\
Sex & 0.858 & $0.393-1.872$ & 0.7 \\
Diabetes & 1.113 & $0.372-3.327$ & 0.56 \\
Congestive heart failure & 0.431 & $0.119-1.562$ & 0.23 \\
Baseline eGFR (per ml/min/1.73m²) & 0.995 & $0.978-1.013$ & 0.616 \\
Iodinated contrast volume (per ml) & 1 & $0.996-1.005$ & 0.868 \\
CTA within 48 hours post EVAR & 1.125 & $0.527-2.402$ & 0.761 \\
Renal artery occlusion & 1.371 & $0.774-2.790$ & 0.384 \\
Acute Kidney Injury & 3.013 & $1.563-5.808$ & 0.001 \\
Procedure time (per minute) & 1.001 & $0.997-1.004$ & 0.707
\end{tabular}

Figure 1 shows the distribution of eGFR categories in all patients over time. Figure 2 illustrates the mean eGFR measurements over time, for subgroups with and without AKI and renal artery occlusions, respectively. Both groups start at similar baseline mean eGFR. For patients with AKI eGFR levels decrease sharply during 48 hours following complex EVAR and show substantial recovery at discharge.

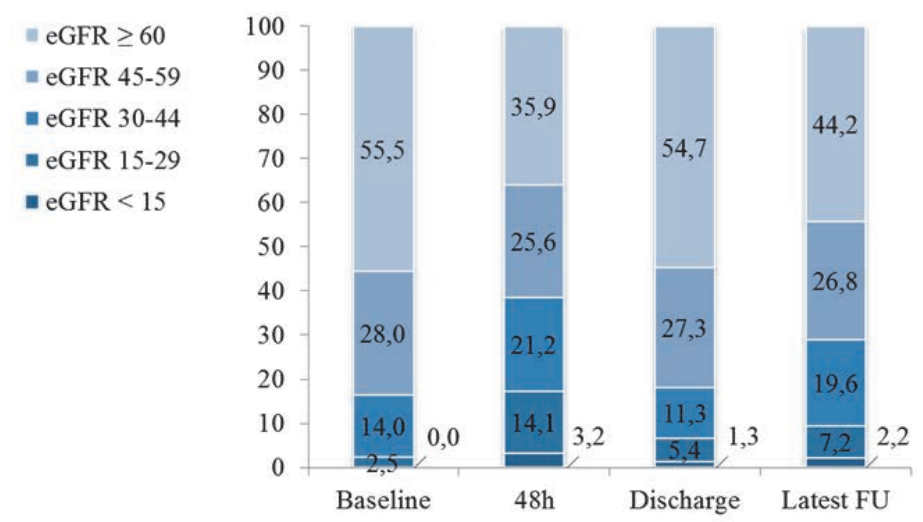

Figure 1. Percentage of patients per eGFR category at baseline, during $48 \mathrm{~h}$ following EVAR, at discharge and latest follow-up (FU). 
At the end of follow-up eGFR levels are substantially lower than at baseline for patients who developed AKI, whereas patients without AKI show only minor decrease in mean eGFR levels at end of follow-up when compared with baseline.

\subsection{DISCUSSION}

This study presents a retrospective evaluation of renal function changes after intraarterial administration of high volumes of low-osmolar iodinated contrast media in a large patient population. Well documented pre- and post-interventional renal function testing and thorough follow-up allowed us to evaluate the renal function in the post-operative period as well as beyond discharge of the patients. In multivariate regression analyses, several risk factors were evaluated regarding their association with post-operative renal function decrease. We discuss the most important findings with respect to the design of the study and current literature.

\section{Influence of intra-arterial iodinated contrast and procedure time on short-and longterm renal function}

For post-operative renal function decrease, which we term 'post-EVAR AKI' throughout the manuscript, we used the definition from the Acute Kidney Injury Network (AKIN) to assess post-EVAR AKI in order to provide a commonly used definition of AKI in research and clinical practice. ${ }^{13-16}$ Another definition of contrast material-induced nephropathy (CIN) uses SCr increase of $\geq 0.5 \mathrm{mg} / \mathrm{dl}$ and / or $\geq 25 \%$ above baseline within 72 hours post intervention. ${ }^{17,18}$ When applying the $\mathrm{CIN}$ criteria for AKI, the patient group who developed post-EVAR AKI would have been the same in our study, excluding two patients that were not included when applying the AKIN criteria. AKIN criteria were used in our study for two main reasons: (1) we followed a recently advocated recommendation from the American College of Radiology in order to standardize the various used definitions of $\mathrm{CIN}^{19}$ (2) postEVAR AKI might not be due to contrast medium but might predominantly be influenced by other factors rather than low-osmolar contrast volume. ${ }^{7}$

With respect to the long-lasting discussion on the toxicity of iodinated contrast material ${ }^{20-}$ 22 , we made two observations: First iodinated contrast volume was associated with a slightly increased risk for every millilitre of contrast applied, however it was not an independent risk factor for AKI and long.-term renal function decrease post EVAR in complex aneurysms. Although univariate analysis suggested a significant effect of contrast volume for the development of AKI, this effect disappeared after adjustment for procedure time. This finding corroborates the hypothesis that high contrast volume is a marker for complexity of the procedure and that patients undergoing more complex procedures have higher risk for renal function decrease. One has to be aware that in any 


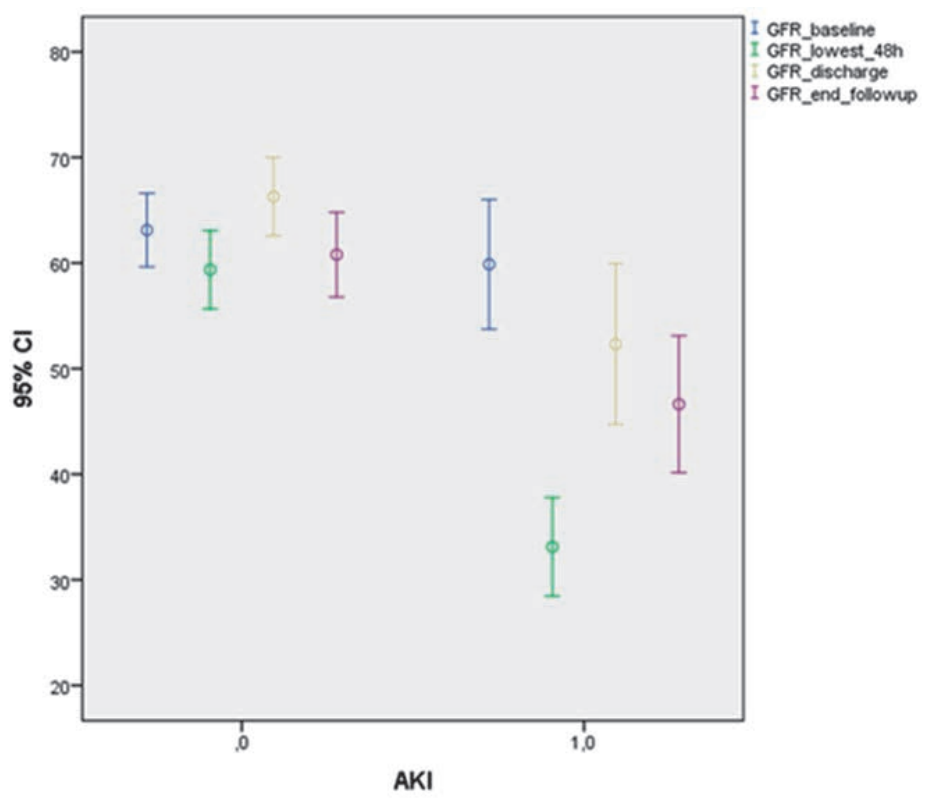

Figure 2. Mean eGFR levels with $95 \%$ confidence interval at baseline, during 48 h post EVAR (lowest level), at hospital discharge and at latest follow-up in patients undergoing complex EVAR. Comparison of eGFR levels in patients without acute kidney injury (coded as $0 ; n=113$ ) and with acute kidney injury (coded as $1 ; n=43$ ).

retrospective study, observed associations are not to be confused with causative relationships. Although we found a strong association between procedure time and short-term renal function decrease, a causal relationship for procedure time itself is hereby not proven. Second, it has been questioned whether post-interventional decrease in renal function is more likely a transient subclinical ${ }^{23,24}$ or even artificial ${ }^{25,26}$ short-term phenomenon, or indeed a relevant predictor for long-term renal dysfunction. When looking at the mid- and long-term renal function measurements we made an interesting observation. While presence of AKI was a significant predictor for eGFR decrease at discharge and last follow-up, the data provide no evidence that procedure time or other factors were significantly associated with eGFR decrease beyond 48 hours. Although no significant associations were found with the other risk factors, directions of the hazards ratios were conform expectations for older age, diabetes and baseline eGFR. Lack of statistical significance may be due to the limited sample size.

\section{Accessory renal artery coverage}

Accessory renal artery coverage and other iatrogenic renal artery side branch occlusion with consecutive visible parenchymal perfusion defect on angiogram or follow-up CTA was a significant predictor for eGFR decrease in the immediate post-operative period. 
Any new parenchymal perfusion defect identifiable on angiogram or CTA therefore is an indicator for short-term decrease of renal function. At discharge and latest follow-up, this risk factor was still associated with increased risk of eGFR decrease (HR = 1.970 and $H R=$ 1.371, respectively) but this association was no longer statistically significant ( $p=0.106$ and $p=0.384$, respectively). This finding is in line with the results from a non-randomized retrospective study, where no significant long-term renal deterioration was found in patients with accessory renal artery coverage during EVAR compared to a control group. ${ }^{27}$ In the long-term, function of the focal parenchymal mass loss seems to be maintained by the remaining renal tissue. In our study, 28 cases of accessory renal artery coverage or renal artery side branch injures occurred, of which only two cases did not show any apparent parenchymal infarction on angiographic imaging and therefore were not included in this category. Beyond registration of infarction, no attempt was made to further quantify the diameter of the occluded renal arteries and the volume of the renal tissue defect. In accordance to a Society of Vascular Surgery consensus statement on the treatment of abdominal aortic aneurysms ${ }^{28}$, at our institution only accessory arteries smaller than or equal to three millimetres in diameter and supporting not more than one third of the renal parenchyma are regularly overstented. This approach seems a relatively safe method at least for the long-term renal function. For larger accessory renal arteries, we cannot infer definite conclusions from our data, and any attempt to preserve their flow (e.g. with additional fenestrations) might be favourable.

In conclusion, intra-arterial iodinated contrast volume is not an independent risk factor for development of short- or long-term renal function decrease after endovascular repair of complex aortic aneurysms. Patients who develop post-EVAR AKI are at significant higher risk for long-term renal function decrease.

\section{ACKNOWLEDGEMENT}

The authors want to thank Ms Stella Schreurs, MPH, for her help with data collection.

\section{REFERENCES}

1. United Kingdom EVAR Trial Investigators, Greenhalgh RM, Brown LC, Powell JT, Thompson SG, Epstein D, Sculpher MJ. Endovascular versus open repair of abdominal aortic aneurysm. N Engl J Med 2010; 362(20):186371.

2. Walsh SR, Tang TY, Sadat U, Naik J, Gaunt ME, Boyle JR et al. Endovascular stenting versus open surgery for thoracic aortic disease: systematic review and meta-analysis of perioperative results. J Vasc Surg 2008;47(5):1094-1098.

3. Verhoeven EL, Katsargyris A, Bekkema F, Oikonomou K, Zeebregts CJ, Ritter W, Tielliu IF. Ten-year Experience with Endovascular Repair of Thoracoabdominal Aortic Aneurysms: Results from 166 Consecutive 


\section{CHAPTER 6}

Patients. Eur J Vasc Endovasc Surg Jan 172015 pii: S1078-5884(14)00649-2. doi: 10.1016/j.ejvs.2014.11.018. [Epub ahead of print].

4. Azuma T, Yokoi Y, Yamazaki K. The next generation of fenestrated endografts: results of a clinical trial to support an expanded indication for aortic arch aneurysm treatment. Eur J Cardiothorac Surg 2013;44(2):e15663; discussion e163.

5. Sailer AM, de Haan MW, Peppelenbosch AG, Jacobs MJ, Wildberger JE, Schurink GW. CTA with fluoroscopy image fusion guidance in endovascular complex aortic aneurysm repair. Eur J Vasc Endovasc Surg 2014;47(4):349-56.

6. de Ruiter QM, Moll FL, van Herwaarden JA. Current state in tracking and robotic navigation systems for application in endovascular aortic aneurysm repair. J Vasc Surg 2015;61(1):256-64.

7. Davenport MS, Cohan RH, Khalatbari S, Ellis JH. The challenges in assessing contrast-induced nephropathy: where are we now? AJR Am J Roentgenol 2014;202(4):784-9.

8. Lee JT, Varu VN, Tran K, Dalman RL. Renal function changes after snorkel/chimney repair of juxtarenal aneurysms. J Vasc Surg 2014;60(3):563-70.

9. Saratzis A, Sarafidis P, Melas N, Khaira H. Comparison of the impact of open and endovascular abdominal aortic aneurysm repair on renal function. J Vasc Surg 2014;60(3):597-603.

10. Karthikesalingam A, Bahia SS, Patel SR, Azhar B, Jackson D, Cresswell L, Hinchliffe RJ, Holt PJ, Thompson MM. A systematic review and meta-analysis indicates underreporting of renal dysfunction following endovascular aneurysm repair. Kidney Int 2015;87(2):442-51.

11. Levey AS, Coresh J, Greene T, Stevens LA, Zhang YL, Hendriksen S, Kusek JW, Van Lente F; Chronic Kidney Disease Epidemiology Collaboration. Using standardized serum creatinine values in the modification of diet in renal disease study equation for estimating glomerular filtration rate. Ann Intern Med 2006;145(4):247-54.

12. Mehta RL, Kellum JA, Shah SV, Molitoris BA, Ronco C, Warnock DG, Levin A; Acute Kidney Injury Network. Acute Kidney Injury Network: report of an initiative to improve outcomes in acute kidney injury. Crit Care 2007;11(2):R31.

13. McDonald RJ, McDonald JS, Bida JP, Carter RE, Fleming CJ, Misra S, Williamson EE, Kallmes DF. Intravenous contrast material-induced nephropathy: causal or coincident phenomenon? Radiology 2013;267(1):106-18.

14. Davenport MS, Khalatbari S, Cohan RH, Dillman JR, Myles JD, Ellis JH. Contrast material-induced nephrotoxicity and intravenous low-osmolality iodinated contrast material: risk stratification by using estimated glomerular filtration rate. Radiology 2013;268(3):719-28.

15. Davenport MS, Khalatbari S, Cohan RH, Ellis JH. Contrast medium-induced nephrotoxicity risk assessment in adult inpatients: a comparison of serum creatinine level- and estimated glomerular filtration rate-based screening methods. Radiology 2013;269(1):92-100.

16. Ehrmann S, Badin J, Savath L, Pajot O, Garot D, Pham T, Capdevila X, Perrotin D, Lakhal K. Acute kidney injury in the critically ill: is iodinated contrast medium really harmful? Crit Care Med 2013;41(4):1017-26.

17. Waikar SS, Liu KD, Chertow GM. Diagnosis, epidemiology and outcomes of acute kidney injury. Clin J Am Soc Nephrol 2008;3(3):844-61.

18. Stacul F, van der Molen AJ, Reimer P, Webb JA, Thomsen HS, Morcos SK, Almén T, Aspelin P, Bellin MF, Clement O, Heinz-Peer G; Contrast Media Safety Committee of European Society of Urogenital Radiology (ESUR). Contrast induced nephropathy: updated ESUR Contrast Media Safety Committee guidelines. Eur Radiol 2011;21(12):2527-41. 
19. American College of Radiology Committee on Drugs and Contrast Media. Manual on contrast media. 9th ed. Reston, Va. http://www.acr.org/ /media/ACR/Documents/PDF/QualitySafety/Resources/Contrast\%20 Manual/2013_Contrast_Media.pdf. Accessed March 172015.

20. Tepel M, Aspelin P, Lameire N. Contrast-induced nephropathy: a clinical and evidence-based approach. Circulation 2006;113(14):1799-806.

21. Davenport MS, Cohan RH, Khalatbari S, Ellis JH. The challenges in assessing contrast-induced nephropathy: where are we now? AJR Am J Roentgenol 2014;202(4):784-9.

22. Liu YH, Liu Y, Tan N, Chen JY, Zhou YL, Luo JF, Yu DQ, Li LW, Li HL, Ye P, Ran P. Contrast-induced nephropathy following chronic total occlusion percutaneous coronary intervention in patients with chronic kidney disease. Eur Radiol 2015;25(8):2274-81.

23. Baumgarten DA, Ellis JH. Contrast-induced nephropathy: contrast material not required? AJR Am J Roentgenol 2008;191(2):383-6.

24. Hayakawa K, Tanikake M, Kirishima T, Yoshinami N, Shintani H, Yamamoto E, Morimoto T. The incidence of contrast-induced nephropathy (CIN) following transarterial chemoembolisation (TACE) in patients with hepatocellular carcinoma (HCC). Eur Radiol 2014;24(5):1105-11.

25. Stratta P, Izzo C, Canavese C, Quaglia M. Letter to the Editor re: Are intravenous injections of contrast media really less nephrotoxic than intra-arterial injections? Eur Radiol 2013;23(5):1260-3.

26. Katzberg RW, Newhouse JH. Intravenous contrast medium-induced nephrotoxicity: is the medical risk really as great as we have come to believe? Radiology 2010;256(1):21-8.

27. Greenberg JI, Dorsey C, Dalman RL, Lee JT, Harris EJ, Hernandez-Boussard T, Mell MW. Long-term results after accessory renal artery coverage during endovascular aortic aneurysm repair. J Vasc Surg 2012;56(2):2916; discussion 296-7.

28. Chaikof EL, Brewster DC, Dalman RL, Makaroun MS, Illig KA, Sicard GA, Timaran CH, Upchurch GR Jr, Veith FJ. SVS practice guidelines for the care of patients with an abdominal aortic aneurysm: executive summary. J Vasc Surg 2009;50(4):880-96. 



\title{
CHAPTER
}

Fusion image guidance in endovascular peripheral artery interventions - a feasibility study

\author{
A.M. Sailer \\ M.W. de Haan \\ R. de Graaf \\ W.H. van Zwam \\ G.W.H. Schurink \\ P.J. Nelemans \\ J.E.Wildberger JE \\ M. Das
}

Cardiovascular and Interventional Radiolology

2015 Apr;38(2):314-21 


\section{ABSTRACT}

Objective: To evaluate the feasibility of endovascular guidance by means of live fluoroscopy fusion with magnetic resonance angiography (MRA) and computed tomography angiography (CTA).

Methods: Fusion guidance was evaluated in twenty endovascular peripheral artery interventions in seventeen patients. Fifteen patients had received pre-interventional diagnostic MRA and two patients had undergone CTA. Time for fluoroscopy with MRA/CTA co-registration was recorded. Feasibility of fusion guidance was evaluated according to following criteria: For every procedure the executing interventional radiologists recorded whether 3D road-mapping provided added value (yes versus no) and whether PTA and/or stenting could be performed relying on the fusion road-map without need for diagnostic contrast enhanced angiogram series (CEAS)(yes versus no). Precision of the fusion road-map was evaluated by recording maximum differences between the position of the vasculature on the virtual CTA/MRA images and conventional angiography.

Results: Average time needed for image co-registration was $5 \pm 2$ minutes. 3D road-map added value was experienced in fifteen procedures in twelve patients. In half of the patients (8/17), intervention was performed relying on the fusion road-map only, without diagnostic CEAS. In two patients MRA roadmap showed a false positive lesion. Excluding three patients with inordinate movements, mean difference in position of vasculature on angiography and MRA/CTA road-map was $1.86 \pm 0.95 \mathrm{~mm}$, implying that about $95 \%$ of differences were between 0 and $3.72 \mathrm{~mm}(2 \pm 1.96 * \mathrm{sd})$.

Conclusion: Fluoroscopy with MRA/CTA fusion guidance for peripheral artery interventions is feasible. By reducing the number of CEAS, this technology may contribute to enhance procedural safety. 


\subsection{INTRODUCTION}

Symptomatic peripheral artery disease (PAD) affects more than ten million people worldwide. ${ }^{1,2}$ Percutaneous transluminal angioplasty (PTA) with or without stent placement is a minimally invasive treatment option to restore arterial blood flow without need for open surgery. Contrast enhanced magnetic resonance angiography (MRA) has gained broad acceptance in the pre-interventional work-up of patients and is routinely performed to diagnose PAD and to determine suitable treatment. If MRA is contraindicated or not available, contrast enhanced computed tomography (CTA) is performed. During endovascular interventional procedures, diagnostic angiography with iodinated contrast medium (CM) is traditionally used in order to facilitate safe maneuvering of guide wires and catheters and to correctly position angioplasty balloons and stents. Over the last decade, MR guided interventions and co-registration of different imaging modalities have been promoted in order to facilitate multimodality fusion image road-mapping. ${ }^{3-7}$ Recently it has been shown that fusion image road-mapping can significantly reduce procedural CM volume in aneurysm repair and liver embolization. ${ }^{8-12}$ To the best of our knowledge, fusion guidance in peripheral artery interventions has not been reported so far. Aim of this study was to evaluate the feasibility, precision and added value of endovascular guidance by means of live fluoroscopy fusion with MRA and CTA.

\subsection{MATERIALS AND METHODS}

The study was approved by the Institutional Ethical Committee, written informed consent was waived (METC 12-4-125). Fusion image road-mapping technology was evaluated in twenty endovascular peripheral artery interventions in seventeen patients. Fifteen patients had undergone outpatient contrast-enhanced MRA of the iliac and lower leg arteries for diagnostic workup as published elsewhere ${ }^{13}$ (Intera 1.5T, Philips Healthcare, Best, The Netherlands; 2D bolus tracking method, $15 \mathrm{ml}$ Gadubotrol [Gadovist, Bayer Healthcare, Berlin, Germany; 7 ml Gd@1.2 ml/s-8.0 ml Gd@0.5 ml/s -followed by a saline chaser of $20 \mathrm{ml} \mathrm{NaCl} @ 0.5 \mathrm{ml} / \mathrm{s}]$ ). In two patients with contraindications for MRA, contrast-enhanced CTA was performed (Somatom Definition, Siemens Healthcare, Forchheim, Germany; scan protocol: 2x64x0.6 mm coll., pitch 0.9, 100/140kV, quality ref. $\mathrm{mAs}$ 136/116 (+CareDose4D), rot. time 0.33s, 1.0/0.8 mm slice reconstruction, soft tissue filter (D30f), $96 \mathrm{ml}$ iopromide 300 (Ultravist, Bayer, Berlin, Germany; [flow rate $4.8 \mathrm{ml}$, IDR $=1.44 \mathrm{gl} / \mathrm{s}]$ ). Timing interval between the MRA/ CTA acquisition and endovascular procedure was up to eight weeks.

Volumetric datasets of the pre-procedurally acquired MRA and CTA images were loaded to a commercially available 3D image fusion workstation (Allura XtraVision 8.3, Philips). Patients were placed in a supine position on the angiography unit's table comparable to 
the position during MRA and CTA acquisition. Flat panel detector C-arm cone-beam CT (CBCT) (Allura Xper FD20, Philips) of the pelvic or upper leg region was acquired right before draping the patient. Volumetric $\mathrm{CBCT}$ datasets were automatically transferred to the 3D workstation. Co-registration of the pre-acquired MRA/ CTA datasets to the CBCT datasets was performed manually by the interventional radiologists. Figure 1 shows the procedure of dataset co-registration.

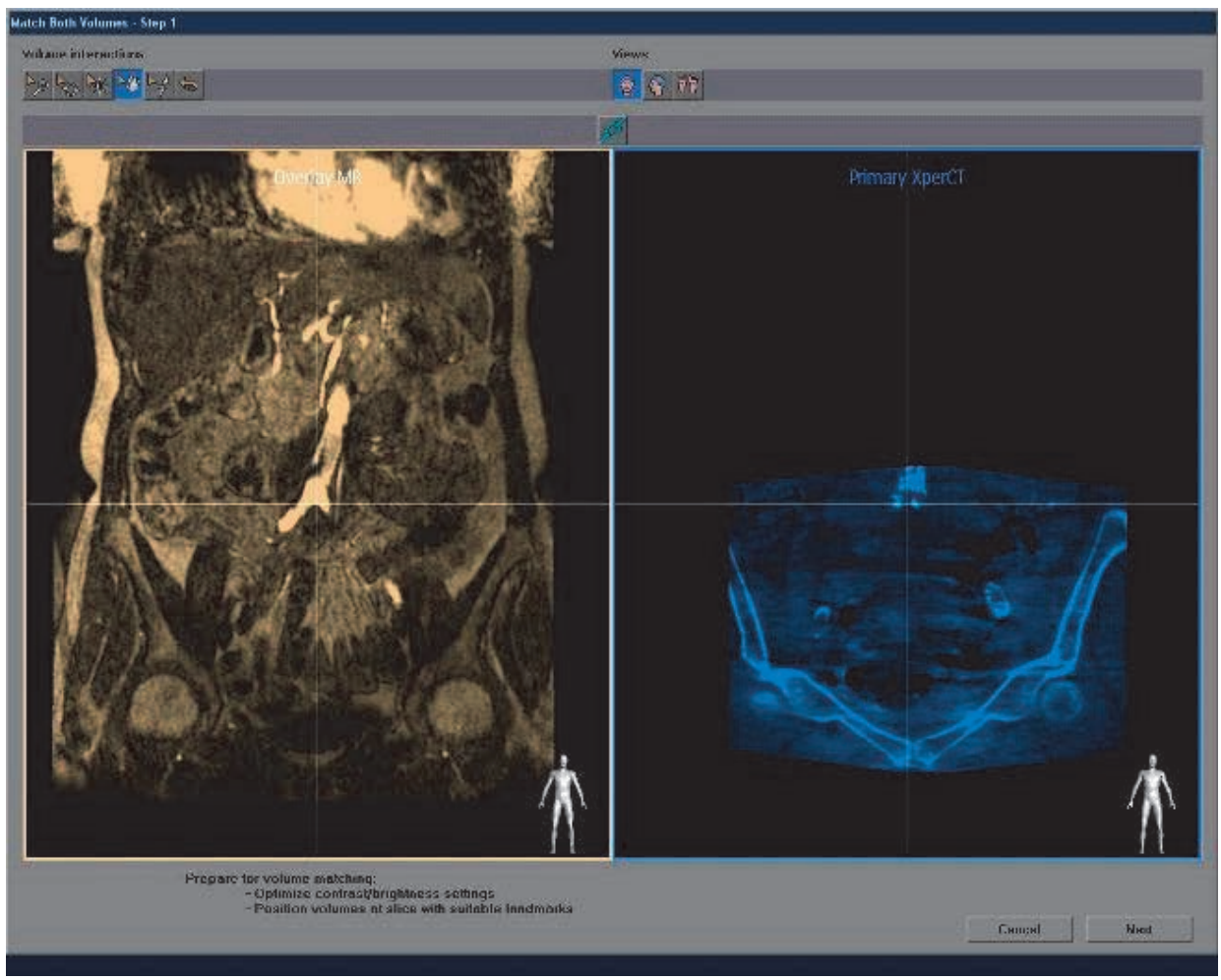

Figure 1. Manually performed rigid coregistration of the volumetric contrast-enhanced MRA dataset (left) and $\mathrm{CBCT}$ dataset (right) by bringing aortic bifurcation and corresponding vessel wall calcifications into the crosshair. MRA and CBCT image coregistration is further adjusted in axial and sagittal planes (not shown) using vessel borders, bone structures, and muscles as landmarks.

Vessels, corresponding vessel wall calcifications, bones and organ borders visible on both datasets were used as landmarks for registration and were verified in three dimensions. By these means, the MRA/ CTA datasets were linked to the 3D coordinate space of the Carm and the corresponding live fluoroscopy image. Dataset co-registration persisted the entire procedure; the MRA/ CTA volume thereby followed any rotation of the C-arm as well as table movements and is adapted to magnification. Guidewires, catheters, PTA balloons and stents were overlaid to the MRA/ CTA vessel tree background allowing non- 
contrast enhanced endovascular road-mapping. By means of manually window leveling, the vasculature of the MRA dataset could be made clearly visible with reduced artifacts from bone and soft tissue structures. Bone-removal enhanced the vessel tree on the CTA dataset.

\section{Patients and Procedures}

Fluoroscopy with MRA/ CTA fusion road-map technology was evaluated in fourteen common and/ or external iliac artery interventions and six superficial femoral artery and/ or popliteal artery interventions in seventeen patients. Patients with below-the-knee lesions and patients that had undergone diagnostic imaging longer than eight weeks ago were not included. Interventions were performed under local anesthesia. A detailed description of the included patients, clinical presentations and vascular pathology on imaging is given in Table 1. Figures 2 to 4 show application of fusion road-mapping in various procedures.

Table 1. Overview of included patients and their clinical presentation and corresponding contrast-enhanced MRA and CTA findings

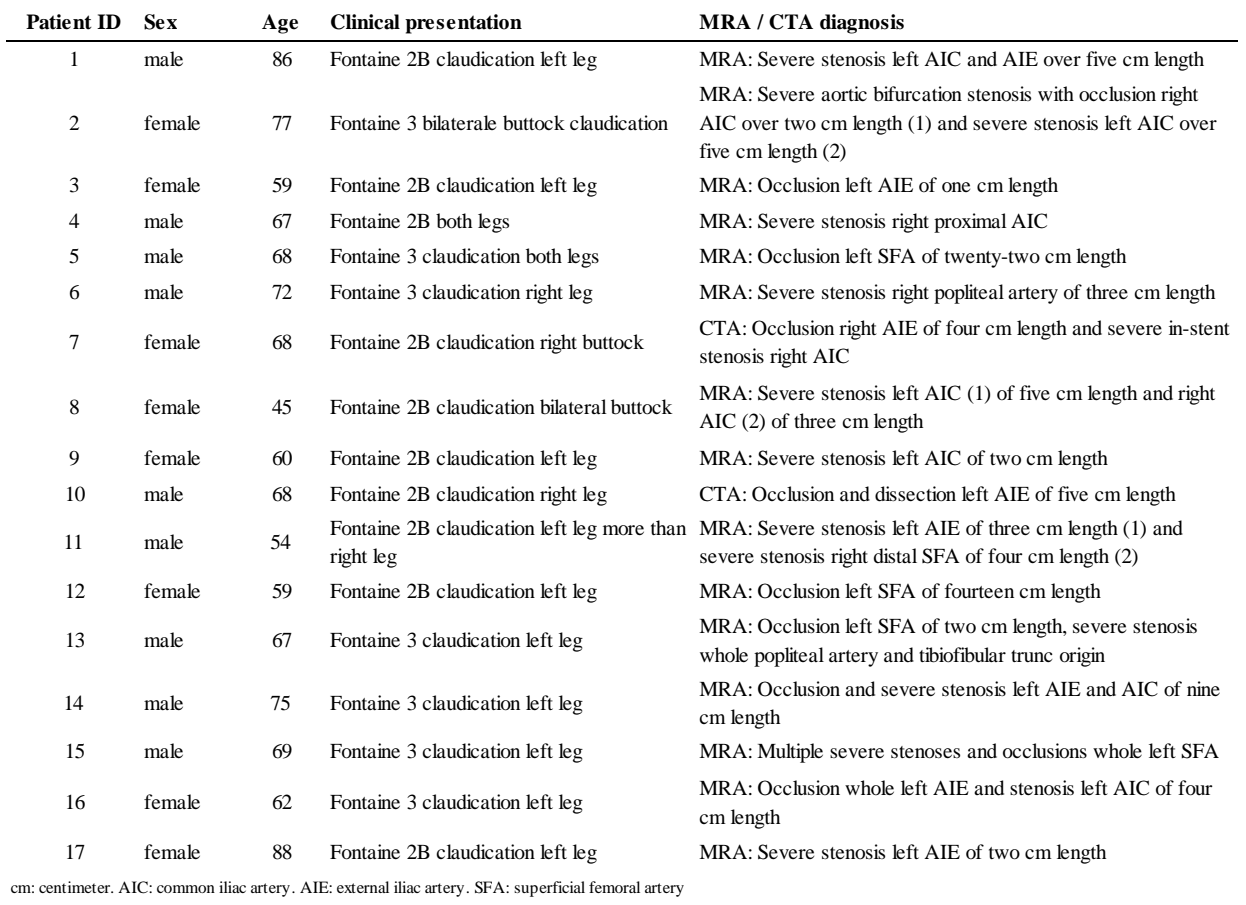




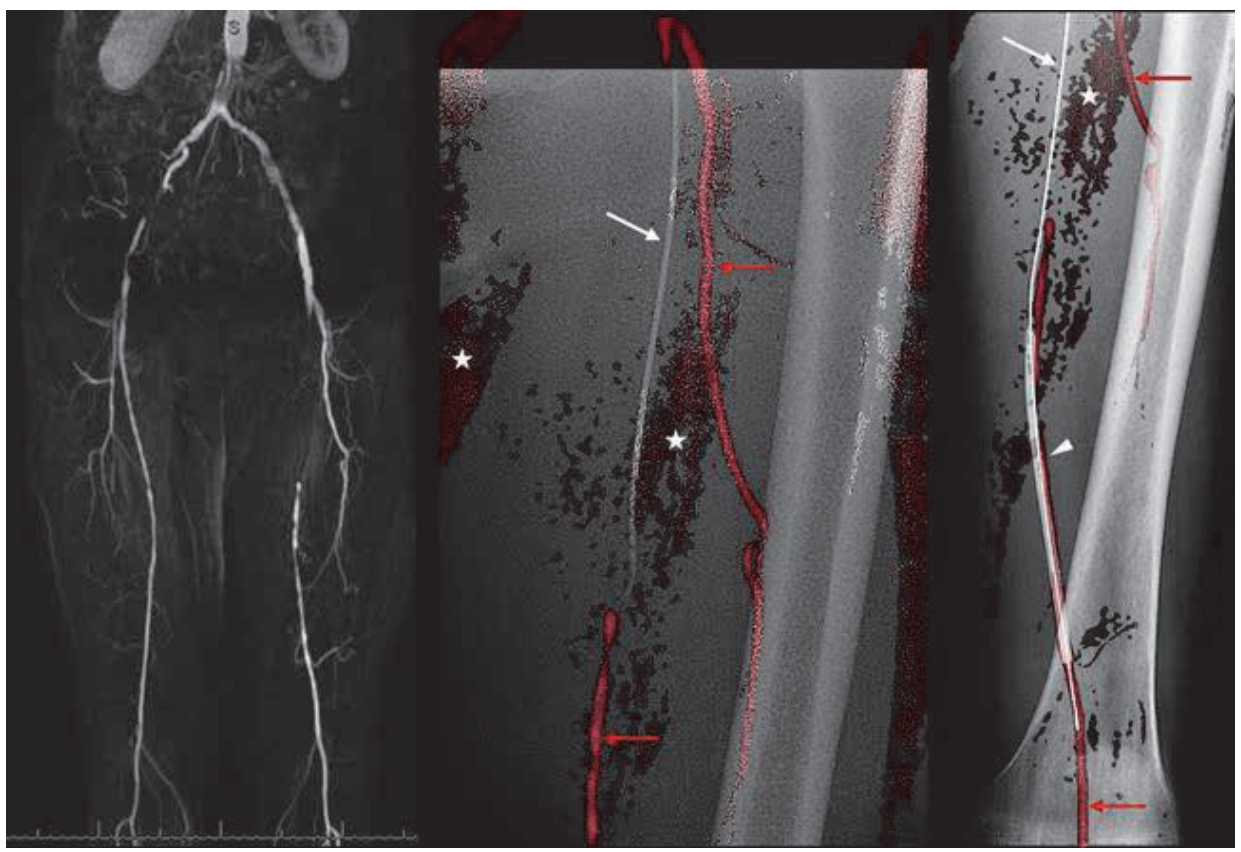

Figure 2. Contrast-enhanced MRA image (left) in patient 12 shows long distance occlusion of the left proximal superficial femoral artery (SFA). Artifacts from previously placed stent in the distal aorta and occlusion of the right external iliac artery are visible. Live fluoroscopy overlay (grey) to the MRA dataset (background) allows noncontrast usage 3D road mapping during SFA recanalization (middle) and balloon angioplasty (right). Guidewire (white arrows) and PTA balloon (white arrow head) superimposed on the MRA vessel tree (red arrows). Artifacts from upper leg muscles (stars), partly removed from the MRA dataset by window-leveling.

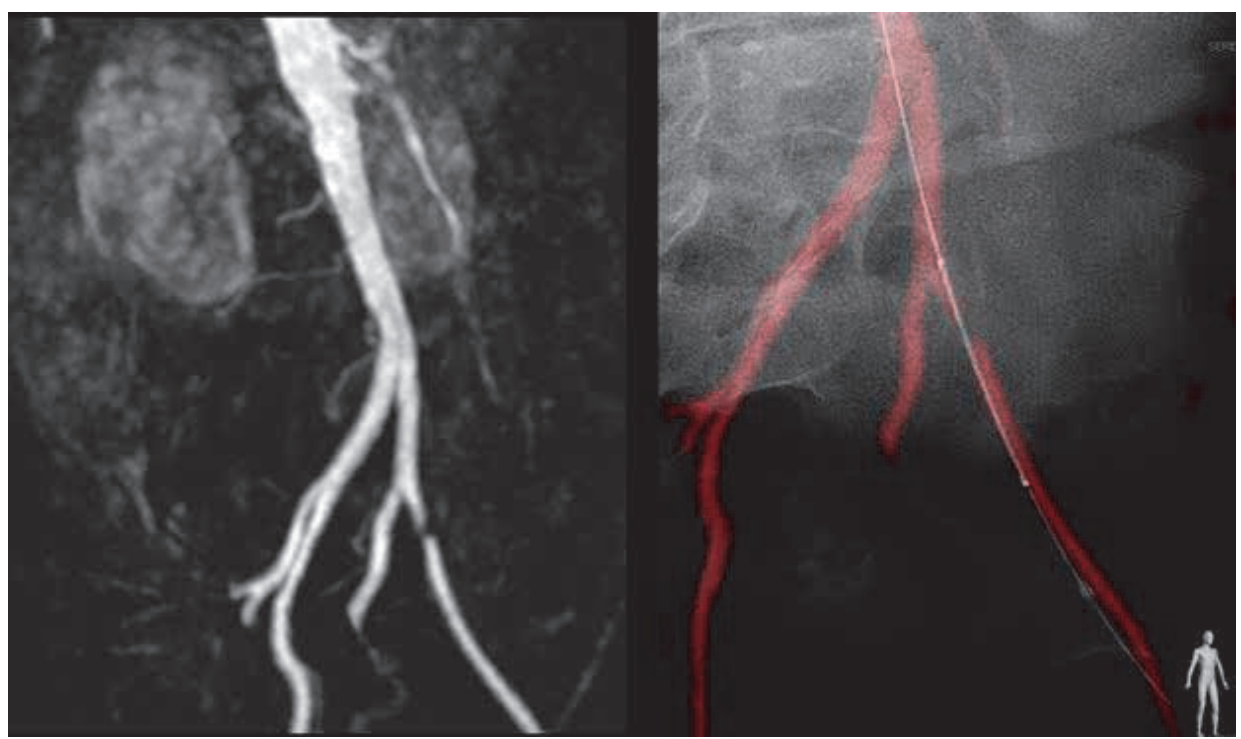

Figure 3. Contrast-enhanced MRA image (left) in patient 3 shows a short occlusion of the left AlE. Direct retrograde access for left external iliac artery recanalization and balloon angioplasty based on fusion road- 
mapping without further need of CM. C-arm in 40 degrees right lateral position and fusion road-map following the 3D projection of live fluoroscopy. Conventional post-PTA control angiography was performed and indicated need for additional stent placement.

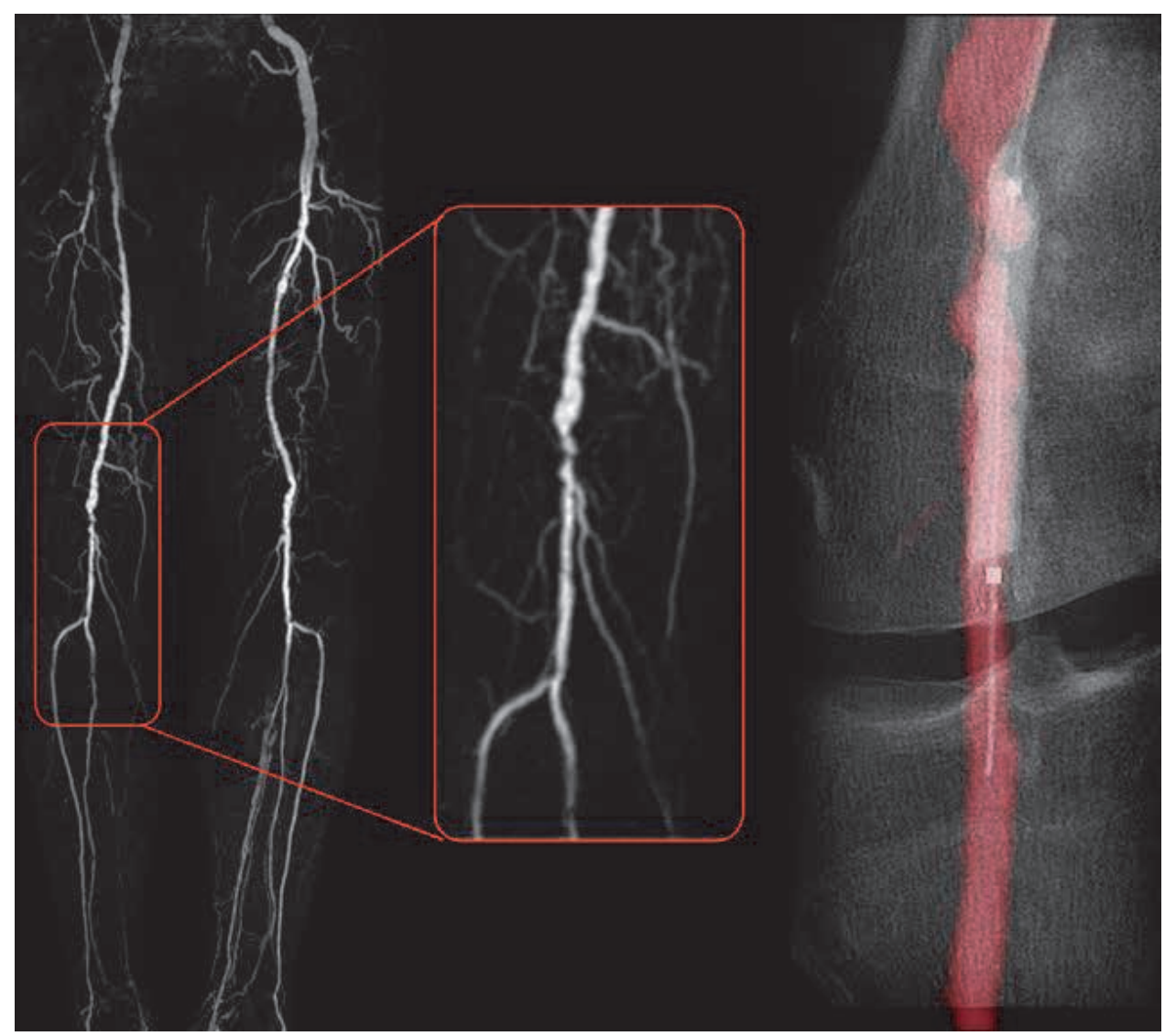

Figure 4. Contrast-enhanced MRA image (left) in patient 6 shows a stenosis of the right popliteal artery. PTA balloon positioning and deployment based on the fusion road-map (right) without need for additional diagnostic CM-enhanced angiogram.

\section{Fusion road-map evaluation}

Time for C-arm CBCT with MRA/ CTA dataset co-registration (in minutes) was monitored. Feasibility of fusion guidance was evaluated according to the following criteria: 1 . For every procedure the executing interventional radiologists recorded whether 3D roadmapping provided added value in terms of defining the best C-arm projection angle for the particular intervention (yes versus no) and whether PTA and/ or stenting could be performed relying on the fusion road-map without the need for a diagnostic contrast enhanced angiogram series (CEAS) (yes versus no). Subjective scores were documented by the interventional radiologists using a standardized online data registration form right 
after the procedure. 2. Precision of the MRA/ CTA with fluoroscopy fusion road-map was evaluated by recording differences between the position of the vasculature on the virtual MRA/ CTA images and conventional angiography. Displacement of the fusion road map was measured as the maximum mismatch in the region of interest (ROI) in millimetres using the measurement tool of the 3D workstation subsequently to each procedure. In patients in whom there was no need for performing a diagnostic angiogram before treatment, the fusion roadmap was compared with the control angiogram that was made after treatment.

\subsection{RESULTS}

Average time needed for dataset co-registration was $5 \pm 2$ minutes (mean \pm standard deviation). Fusion road-map added value was experienced in fifteen procedures (75\%) in twelve patients. In three procedures, inordinate patient movement after CBCT acquisition hindered the use of the fusion road-map. In the procedures of patients no. 4 and 17, the diagnostic MRA and corresponding fusion road-map indicated a severe stenosis of the right common iliac artery (AIC) and left external iliac artery (AIE), respectively. The vessel section could be easily passed with the guidewire and conventional angiography was performed to visualize the extent of the stenosis. In both patients, on two directional conventional angiography series, there was no stenosis visible and no intervention was performed. In $47 \%$ of the patients (8 / 17) PTA and/ or stenting could be performed relying on the fusion road-map and a diagnostic CEAS was not required. Average maximum difference in position of vasculature on angiography and MRA/ CTA fusion road-map was $6.41 \mathrm{~mm}$ with a standard deviation of $11.12 \mathrm{~mm}$. Excluding the three patients with major leg and pelvis movement during the procedure, average maximum difference was $1.86 \pm 0.95 \mathrm{~mm}$, which implies that about $95 \%$ of differences were between 0 and $3.72 \mathrm{~mm}(2 \pm 1.96 *$ standard deviation). Distribution of $3 \mathrm{D}$ road-map added value and precision per patient is shown in Table 2.

\subsection{DISCUSSION}

Fusion guidance is a new technology for endovascular road-mapping. Flat panel detectors that can acquire rotational volumetric datasets for image co-registration are increasingly replacing conventional fluoroscopy units and co-registration software is commercially available from different vendors. To our knowledge, we are the first to report fluoroscopy co-registration with MRA/ CTA in the application of peripheral artery interventions. In our series, dataset co-registration with MRA or CTA was feasible in all procedures. Coregistration was performed by the interventional radiologist on the $3 \mathrm{D}$ workstation while 
Fusion Image Guidance in Endovascular Peripheral Artery Interventions

Table 2. Overview of characteristics of endovascular procedures

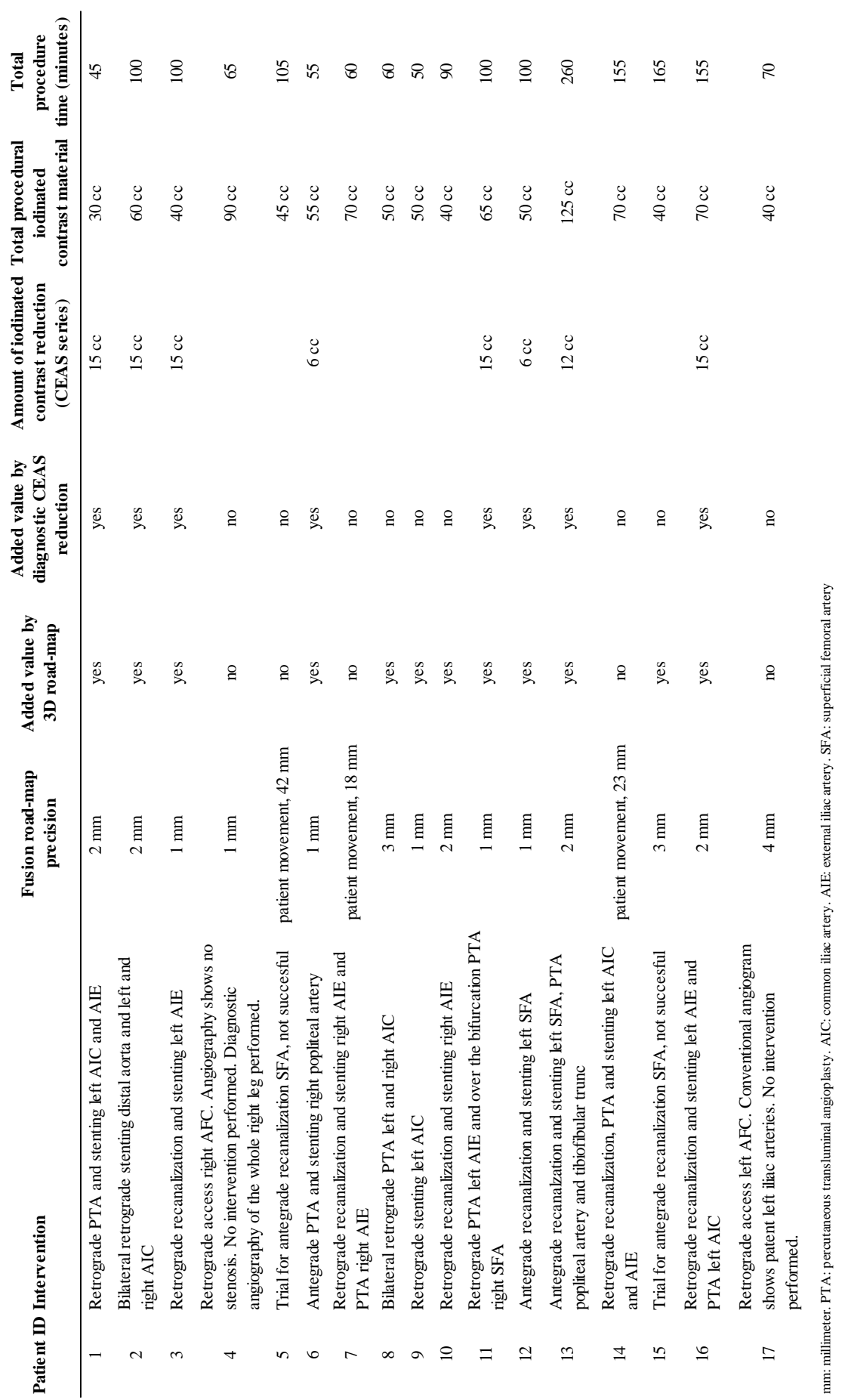


further preparations inside the angiography suite (draping the patient, preparing the table) were carried out by the nurses. Actual time loss for fusion road-mapping was therefore negligible.

Conventional catheter road-mapping with iodinated CM carries potential nephrotoxic side-effects which are correlated with the amount of $\mathrm{CM}$ and should therefore be regarded as relevant in patients with renal impairment and diabetes, both associated with PAD. ${ }^{1,14,15}$ Furthermore, endovascular interventions with angiography are contraindicated in patients with serious allergy to CM. Any technology to reduce procedural CM is therefore of high importance. ${ }^{16}$

Previously, Manke et al. ${ }^{17}$ outlined the feasibility of MR guided iliac artery stenting in thirteen patients. Real-time monitoring by live fluoroscopy was lacking, which resulted in long procedure time. Metal artifacts on MR further restricted applicability for routine clinical practice. These limitations are addressed by fusion technology which links the preinterventional MRA/ CTA information to dynamic fluoroscopy. As volumetric MRA or CTA datasets are registered, the vessel tree follows any three dimensional C-arm rotation. Optimal projection angles can be chosen while rotating the $\mathrm{C}$-arm under fluoroscopy.

In a previously reported case, thoracic aortic repair without $\mathrm{CM}$ administration has been performed. ${ }^{8}$ In our study, PTA and/ or stenting without a pre-treatment CEAS, so fully relying on the fusion road-map, was performed in half of the patients; in the other half, however, we still needed support from an angiogram. Post-treatment control angiograms were further performed in all patients in order to analyze success of the intervention and to detect possible complications; we therefore did not perform procedures without CM administration. Still the reduction of pre-interventional angiograms in eight patients shows that fusion guidance technology holds the potential for CM reduction in future procedures which could be especially relevant for patients with renal impairment. In patients with severe allergy to iodinated contrast material, zero-iodinated-contrast interventions might be possible in combination with ultrasound control or low volume of gadolinium or carbon dioxide. Further trials - ideally prospective randomized trials - are necessary to prove the concept of contrast saving by image fusion and to quantitatively determine effects on procedural contrast usage in a broad patient population.

Dataset co-registration requires acquisition of a CBCT. The staff is usually outside the angiosuite during acquisition, but extra radiation dose for the patient has to be considered. Mean patients' radiation dose in terms of dose are product (DAP) from CBCT in the pelvic region was $14.5 \mathrm{Gycm}^{2}$ (95\% Cl: 11.6; $\left.17.4 \mathrm{Gycm} 2\right)$ which accounted on average for $21.4 \%(95 \% \mathrm{Cl}: 16.4 ; 26.2 \%)$ of the total procedural DAP. Calculated maximum risk for lifetime exposure induced cancer death is less than $0.03 \%$ in pelvic CBCT. ${ }^{18}$ For the PAD patient population, potential benefit from contrast savings by use of fusion road-mapping might overweight the negative impact of the extra radiation dose. 
However, this has to be judged by the interventional radiologist individually for each case under consideration of patient's renal function and age.

Main limitations of fusion road-mapping are patient movement and MRA artifacts. Similar to conventional road mapping, any patient movement after CBCT acquisition results in mismatch of the fusion road-map. In $18 \%$ of the patients ( 3 / 17), inordinate (rotational) movements inhibited the use of the fusion road-map - a limitation that needs to be addressed for further application e.g. by immobilization pelvis and legs casts. Furthermore, different patient positioning in pre-interventional imaging and interventional procedure should be considered as this might have affected fusion roadmap precision. The results of this study indicate that - except for the patients with major movement during the procedure - differences between position of vasculature on angiography and MRA/ CTA fusion road-map did not exceed four millimeters. Most of the procedures were iliac interventions. Displacements of such magnitude might be considered acceptable for adequate balloon and stent positioning in most peripheral artery applications, especially in those with larger vessel regions. As was shown in our study, no complications were detected even in those interventions $(n=8)$ which were performed solely relying on fusion road-mapping. Furthermore, minor displacement of the fusion road-map can be corrected manually during the procedure by readjusting the MRA/ CTA dataset on fluoroscopy landmarks e.g. a catheter placed over the aortic bifurcation and other landmarks visible under fluoroscopy. However, for anatomic regions with smaller vessel diameters, recanalization procedures and stenting nearby side branches, potential risk of device misplacement has to be taken serious and might inhibit application of this technology as long as precision is not further improved. In terms of diagnostic accuracy, one must realize that the fusion image road-map is only as accurate as the underlying MRA or CTA quality as artifacts hamper the usability of the fusion road-map. Also timing intervals between MRA acquisition and PTA/ stenting procedure might cause inaccuracies. In two of the studied patients, the diagnostic MRA indicated a false positive (severe) stenosis of the left iliac artery. This error was detected on an additional conventional angiogram and might have resulted in unnecessary PTA if evaluation with conventional angiogram would not have been performed. However, imaging techniques such as CTA and MRA are constantly improving and the number of pseudo lesions decreases. In a recent meta-analysis by Jens et al. ${ }^{19}$ it was shown that both CTA and MRA are highly accurate with sensitivity of $96 \%$ and $93 \%$ and specificity of $95 \%$ and $94 \%$ respectively, but still potential risk of pseudo lesions always needs to be considered when using fusion road-mapping.

In conclusion, fusion of pre-interventional MRA and CTA datasets with live fluoroscopy for real-time 3D road-mapping in endovascular peripheral artery interventions is feasible. The technology holds potential to reduce contrast dosage and may contribute to enhance renal safety. 


\section{CHAPTER 7}

\section{REFERENCES}

1.Selvin E, Erlinger TP. Prevalence of and risk factors for peripheral arterial disease in the United States: results from the national health and nutrition examination survey, 1999-2000. Circulation. 2004;110(6):738-743.

2. Harris K. The worldwide burden of peripheral artery disease. Inter-society consensus for the management of peripheral artery disease (TASC II). Published 2008. Available at: http://www.radiolodzyinterwencyjni.pl/forms/961.pdf. Accessed November 24, 2013.

3. Raval AN, Karmarkar PV, Guttman MA, Ozturk C, Sampath S, DeSilva R, Aviles RJ, Xu M, Wright VJ, Schenke WH, Kocaturk O, Dick AJ, Raman VK, Atalar E, McVeigh ER, Lederman RJ.Real-time magnetic resonance imagingguided endovascular recanalization of chronic total arterial occlusion in a swine model. Circulation. 2006;113(8):1101-1107.

4. Buecker A, Adam GB, Neuerburg JM, Kinzel S, Glowinski A, Schaeffter T, Rasche V, van Vaals JJ, Guenther RW. Simultaneous real-time visualization of the catheter tip and vascular anatomy for MR-guided PTA of iliac arteries in an animal model. J Magn Reson Imaging. 2002;16(2):201-208.

5. Fink C, Bock M, Umathum R, Volz S, Zuehlsdorff S, Grobholz R, Kauczor HU, Hallscheidt P. Renal embolization: feasibility of magnetic resonance-guidance using active catheter tracking and intraarterial magnetic resonance angiography. Invest Radiol. 2004;39(2):111-119.

6. Klein AJ, Tomkowiak MT, Vigen KK, Hacker TA, Speidel MA, Vanlysel MS, Shah N, Raval AN. Multimodality image fusion to guide peripheral artery chronic total arterial occlusion recanalization in a swine carotid artery occlusion model: unblinding the interventionalist. Catheter Cardiovasc Interv. 2012;80(7):1090-1098.

7. van der Hoeven BL, Schalij MJ, Delgado V. Multimodality imaging in interventional cardiology. Nat Rev Cardiol.2012;9(6):333-346.

8. Kobeiter H, Nahum J, Becquemin JP. Zero-contrast thoracic endovascular aortic repair using image fusion.Circulation.2011;124(11):e280-282.

9. Dijkstra ML, Eagleton MJ, Greenberg RK, Mastracci T, Hernandez A. Intraoperative C-arm cone-beam computed tomography in fenestrated/branched aortic endografting. J Vasc Surg. 2011;53(3):583-590.

10. Sailer AM, de Haan MW, Peppelenbosch AG, Jacobs MJ, Wildberger JE, Schurink GW. CTA with Fluoroscopy Image Fusion Guidance in Endovascular Complex Aortic Aneurysm Repair. Eur J Vasc Endovasc Surg. 2014;47(4):349-356.

11. Sadek M, Berland TL, Maldonado TS, Rockman CB, Mussa FF, Adelman MA, Veith FJ, Cayne NS. Use of preoperative magnetic resonance angiography and the Artis zeego fusion program to minimize contrast during endovascular repair of an iliac artery aneurysm. Ann Vasc Surg.2014;28(1):261.e1-5.

12. Bargellini I, Turini F, Bozzi E, Lauretti D, Cicorelli A, Lunardi A, Cioni R, Bartolozzi C. Image fusion of preprocedural CTA with real-time fluoroscopy to guide proper hepatic artery catheterization during transarterial chemoembolization of hepatocellular carcinoma: a feasibility study. Cardiovasc Intervent Radiol.2013;36(2):526-530.

13. Versluis B, Backes WH, van Eupen MG, Jaspers K, Nelemans PJ, Rouwet EV, Teijink JA, Mali WP, Schurink GW, Wildberger JE, Leiner T. Magnetic resonance imaging in peripheral arterial disease: reproducibility of the assessment of morphological and functional vascular status. Invest Radiol. 2011;46(1):11-24.

14. Romero JM, Bover J, Fite J, Bellmunt S, Dilmé JF, Camacho M, Vila L, Escudero JR. The modification of diet in renal disease 4-calculated glomerular filtration rate is a better prognostic factor of cardiovascular events than classical cardiovascular risk factors in patients with peripheral arterial disease. J Vasc Surg. 2012; 56(5):13241330. 
15. Hölscher B, Heitmeyer C, Fobker M, Breithardt G, Schaefer RM, Reinecke H. Predictors for contrast mediainduced nephropathy and long-term survival: prospectively assessed data from the randomized controlled Dialysis-Versus-Diuresis (DVD) trial. Can J Cardiol. 2008;24(11):845-850.

16. Laskey WK, Jenkins C, Selzer F, Marroquin OC, Wilensky RL, Glaser R, Cohen HA, Holmes DR Jr; NHLBI Dynamic Registry Investigators. Volume-to-creatinine clearance ratio: a pharmacokinetically based risk factor for prediction of early creatinine increase after percutaneous coronary intervention. J Am Coll Cardiol. 2007;50(7):584-590.

17. Manke C, Nitz WR, Djavidani B, Strotzer M, Lenhart M, Völk M, Feuerbach S, Link J.MR imaging-guided stent placement in iliac arterial stenoses: a feasibility study. Radiology 2001;219(2):527-534.

18. Sailer AM, Schurink GWH, Wildberger JE, de Graaf R, van Zwam WH, de Haan MW, Kemerink GJ, Jeukens CRLPN. Radiation exposure of abdominal Cone Beam Computed Tomography. Cardiovasc Interv Radiol 2014 May 6 [Epub ahead of print]. doi: 10.1007/s00270-014-0900-7.

19. Jens S, Koelemay MJ, Reekers JA, Bipat S. Diagnostic performance of computed tomography angiography and contrast-enhanced magnetic resonance angiography in patients with critical limb ischaemia and intermittent claudication: systematic review and meta-analysis. Eur Radiol 2013;23(11):3104-3114. 

CHAPTER

General Discussion 


\section{VALUE AND CURRENT LIMITATIONS OF MULTIMODAL IMAGE FUSION IN ENDOVASCULAR ANEURYSM REPAIR}

Fenestrated and branched endovascular repair are highly personalized procedures and require a detailed knowledge of the patients' individual vascular anatomy for successful planning and execution of the procedure. Multimodal image fusion enables a 3D visualization of the MDCT data during the image guided interventions without additional contrast application. Chapter 2 evaluated the benefits and limitations of multimodal image fusion guidance in endovascular complex aortic aneurysm repair in our clinical center. Image fusion technology proved to significantly reduce iodinated contrast material by a mean volume of $40 \mathrm{cc}$ or $20 \%$ of procedural iodinated contrast compared to equivalent procedures which had been performed without fusion technology. Despite the extra time needed for set up of modality co-registration for image fusion display, procedures which were performed using image fusion technology had a significantly shorter overall procedure time of on average $1.1 \mathrm{~h}$ or $18 \%$ compared to control procedures. A non-significant reduction of on average nine minutes or $15 \%$ of total fluoroscopy time per procedure was observed. Since the first published studies of multimodal fusion in complex endovascular aortic repair in 2011 and 2013 ${ }^{1,2}$ and the study from this thesis, its clinical application and published data have emerged in a rapid pace. Up to now more than 20 studies have been published reporting the use of fusion in complex but also in abdominal or thoracic standard endovascular aortic repair. A metaanalysis on the pooled effects of fusion in endovascular repair is pending. So far published data of seven prospective clinical studies exist that have compared the use of fusion to procedures without fusion. All of them showed a significant reduction in iodinated contrast use by fusion road-mapping. ${ }^{1-7}$ Four of those studies showed a significant reduction in procedural radiation dose by use of fusion ${ }^{4-7}$ and the other three studies showed a trend towards lower radiation dose or fluoroscopy time. ${ }^{1-3}$ Concerning procedure time, four studies showed a significant reduction in procedure time ${ }^{3,5-7}$ by fusion while the other studies showed a trend towards reduction or no reduction. 1,2,4 Despite the strong published evidence for beneficial effects of fusion on procedure characteristics further research has to be performed in order to assess the impact of fusion technology on technical success and clinical outcome of endovascular aortic repair. Therefore the value of multimodal image fusion on procedural technical success, type 1 and 3 endoleaks rate, re-intervention rate and overall clinical outcome of the patients in terms of perioperative mortality, morbidity and postoperative renal function have to be evaluated.

In the second chapter of this thesis, main causes for dataset overlay inaccuracies have been determined. The two predominant causes for MDCT with fluoroscopy image overlay inaccuracies are respiratory movement of the aorta and side branches as well as temporary mismatch during introduction of stiff devices in elongated vessel segments. 
Both sorts of mismatch are based on the rigid co-registration between MDCT and fluoroscopy. The comprehensive analysis of respiratory displacement of the aorta and branches in vascular patients in chapter 3 outlines that respiratory mismatch is fairly predictable and small at the distal descending and abdominal aorta as well as at the level of the origins of visceral and renal arteries. Significant movements up to 15 millimeters in the proximal aorta however might limit the current application of multimodal fusion in proximal thoracic aortic repair and arch repair. lodinated contrast use before any crucial step in aortic prosthesis placement is advocated. ${ }^{8}$ Further research needs to be done on automated motion correction between the MDCT and fluoroscopy data e.g. based on the new coordinates after every iodinated contrast application or current device position. In elongated vessel segments, expected architecture deformation by introduction of stiff devices should be analyzed and incorporated in the fusion image. Finally, fusion image technology by non-rigid deformable display of the fusion roadmap and optical tracking or electromagnetic tracking of the devices and automated deformation algorithms are expected to further improve accuracy of endovascular repair. ${ }^{9}$

\section{PATIENT AND STAFF RADIATION EXPOSURE DURING ENDOVASCULAR COMPLEX AORTIC REPAIR}

The evaluation of radiation exposure in complex endovascular aortic procedures has to respect the different quality and frequency of exposure for patients and physicians or other staff. While patients are likely to receive a higher dose due to direct exposure to the X-ray bundle, they will ideally undergo complex endovascular procedures no more than once in their lifetime. Patient radiation dose concerns related to a single procedure therefore mainly imply deterministic effects like skin erythema from prolonged fluoroscopy in a particular region. ${ }^{10}$ In comparison, the radiation dose of physicians and staff per procedure tends to be lower as it is derived mainly from scatter radiation. However, the cumulative dose derived from repetitive exposure and the significantly higher amount of endovascular procedures performed, substantially increases the individual staff member's work related radiation burden and risk for stochastic effects. ${ }^{11}$ Small effects on procedural dose therefore have to be evaluated in the context of potential cumulative effects in repetitive procedures.

In chapter 4 the additional radiation burden of patients undergoing endovascular complex aortic aneurysm repair with multimodal technology has been evaluated. Patients are exposed to an additional amount of radiation per acquisition of an abdominal CBCT used to operate 3D dataset co-registration. The effective dose has been determined to be 3.5 and $4.3 \mathrm{mSv}$, depending on the exact position of the abdominal CBCT. Associated risk of lethal cancer due to this extra radiation is below $0.02 \%$. The breakeven between additional radiation from fusion and dose from procedural fluoroscopy is 
reached after approximately 7 minutes. A reduction of procedural fluoroscopy time by 7 minutes or more due to fusion therefore equalizes the additional radiation exposure of the $\mathrm{CBCT}$ and can even yield lower radiation exposure for the patient. Additional dose reduction by any decrease of procedural DSA would further amplify this effect. For staff members, the CBCT should not increase the radiation exposure as they ideally leave the angiosuite and stay in the control room during the short time of CBCT acquisition.

Chapter 5 evaluated the radiation exposure to medical staff during standard and complex endovascular aortic repair procedures. The doses measured for the lead physician during complex repair showed on average $0.34 \mathrm{mSv}$. This might be considered low in the context of the annual personal dose limit of $20 \mathrm{mSv}$. However, in some procedures individual staff doses were registered as high as $1 \mathrm{mSv}$ and turned out to be significantly higher during complex procedures compared to standard repair. This justifies the importance and relevance of current and future efforts towards procedural and staff dose assessment and direct feedback systems for employees. Following the principle of keeping doses as low as reasonable achievable (ALARA) multimodal fusion image technology certainly has found its role in procedural dose protection techniques. ${ }^{12,13}$ Potential further research on CBCT could generate protocols of low dose $\mathrm{CBCT}$ that produce just enough image quality for dataset matching. Accuracy and dose effectiveness of co-registration by means of non-rotational 2D fluoroscopic images compared to CBCT based co-registration should further be assessed. Intra-operative post procedural CBCT can be used for final evaluation at completion and replace postoperative MDCT. ${ }^{1}$ Given the limited range and field of view of $\mathrm{CBCT}$, adequate assessment of the whole length of the endograft would demand at least two slightly overlapping acquisitions with the resulting dose basically equalizing the dose of a single MDCT. Nevertheless, intraoperative evaluation using CBCT might be able to detect potential complications intraoperatively and could decrease re-intervention rate which would have to be shown in further research.

\section{IODINATED CONTRAST MEDIA ADMINISTRATION AND RENAL FUNCTION IN COMPLEX ENDOVASCULAR AORTIC REPAIR AND OTHER IMAGE GUIDED INTERVENTIONS}

By its minimal invasive approach, endovascular repair requires not only the above discussed radiation exposure but also demands administration of iodinated contrast media to visualize the arterial vessel structure. Even though the mechanism of renal toxicity is still not fully understood, published data on clinical and experimental studies propose iodinated contrast media to have toxic effects on renal tubular cells. $10 \%$ of all hospital-acquired renal failures are assumed to be caused by iodinated contrast media administration. Patients with diabetes, chronic renal failure, hypertension, advanced age (over 60 years) or use of NSAIDs are of increased risk and incidence at that group might 
be as high 30\%. ${ }^{14}$ The European Society of Urogenital Radiology (ESUR) defines contrastinduced nephropathy (CIN) as a decrease in renal function within 72 hours of receiving iodinated contrast media in the absence of other causes. ${ }^{15}$ Another term more recently used is acute kidney injury (AKI) with slightly different but in essence similar cut-off values. ${ }^{16}$ Patients diagnosed with CIN have an increased morbidity and mortality. It has been shown that patients undergoing complex endovascular repair are at significant risk of post-operative renal damage. ${ }^{17}$

Chapter 6 assessed the incidence and risk factors for renal function decrease in a retrospective cohort of patients undergoing fenestrated and branched endovascular repair. Post-operative kidney injury was diagnosed in $28 \%$ of the procedures and $78 \%$ percent of patients who died within 30 days of the procedure had developed acute kidney injury. Development of kidney injury was found to be associated with a significant increased risk for long term renal function decrease. The procedural contrast media dose was significantly higher in patients who developed kidney injury compared to patients without kidney injury (195 $\pm 88 \mathrm{ml}$ versus $149 \pm 69 \mathrm{ml}$ respectively). However, when adjusting for other procedural factors, long procedure time and macrovascular embolization to renal parenchyma were found the only independent predictors for renal function decline. Nevertheless, iodinated contrast media remained associated with a slightly increased risk of kidney injury for every milliliter of contrast applied. With respect to high costs of prophylaxis and management of renal failure, any preventive effort to reduce potentially harmful contrast agents are of high clinical relevance. This might not only apply to endovascular repair procedures but also to other image guided interventions. Many patients who are referred for vascular interventional procedures are at increased risk for contrast induced injury like patients with diabetes or preexisting renal failure. Furthermore, allergic adverse events occur in around $3 \%$ of cases, and severe adverse reactions in $0.04 \% .{ }^{18}$ Several strategies have been developed to avoid iodinated contrast media. For many years, carbon dioxide (CO2) has been used as alternative contrast agent in interventional procedures. It can however not be applied above the diaphragm, lacks image quality and patients often suffer from injection discomfort. ${ }^{19}$ Gadolinium as an alternative agent is also limited in terms of visibility on fluoroscopy and the maximal volume, which is determined by $0.3 \mathrm{mmol}$ per $\mathrm{kg}$. Furthermore, gadolinium is relatively expensive compared to iodinated contrast media and is not recommended in patients with chronic renal failure. ${ }^{20}$ Sonography guided interventions have disadvantages in certain locations and outcome strongly depends on physician's skills. ${ }^{21}$ Multimodal image fusion technology extents the current repertoire of strategies to avoid iodinated contrast media.

Chapter 7 evaluated feasibility of multimodal fusion of magnetic resonance angiography (MRA) and fluoroscopy in the application of peripheral artery interventions. Despite decreased visibility of bony structures on MR and smaller vessel diameters in iliac and superficial femoral arteries, dataset registration and image fusion proved feasible and 
showed potential to reduce the amount of administered iodinated contrast agent. This has been adopted by other authors using MRA with fluoroscopy fusion for non-iodinated contrast endovascular aortic repair in patients with renal insuffiency. ${ }^{22}$ Zero iodinated contrast interventions have been an ambitious task in recent years and have already been successfully performed in several reported cases. ${ }^{22-25} \mathrm{~A}$ combination of multimodal image fusion technology and other above mentioned alternative vessel visualization techniques can offer safe and realistic strategies to reduce or eliminate iodinated contrast media if needed. ${ }^{26}$

\section{REFERENCES}

1. Dijkstra ML, Eagleton MJ, Greenberg RK, Mastracci T, Hernandez A. Intraoperative C-arm cone-beam computed tomography in fenestrated/branched aortic endografting. J Vasc Surg. 2011 Mar;53(3):583-90.

2. Tacher V, Lin M, Desgranges P, Deux JF, Grünhagen T, Becquemin JP, Luciani A, Rahmouni A, Kobeiter H. Image guidance for endovascular repair of complex aortic aneurysms: comparison of two-dimensional and three-dimensional angiography and image fusion. J Vasc Interv Radiol. 2013;24:1698-1706.

3. Sailer AM, de Haan MW, Peppelenbosch AG, Jacobs MJ, Wildberger JE, Schurink GW. CTA with fluoroscopy image fusion guidance in endovascular complex aortic aneurysm repair. Eur J Vasc Endovasc Surg. 2014 Apr;47(4):349-56.

4. Hertault A, Maurel B, Sobocinski J, Martin Gonzalez T, Le Roux M, Azzaoui R, Midulla M, Haulon S. Impact of hybrid rooms with image fusion on radiation exposure during endovascular aortic repair. Eur J Vasc Endovasc Surg. 2014 Oct;48(4):382-90.

5. Dias NV, Billberg H, Sonesson B, Törnqvist $P$, Resch $T$, Kristmundsson $T$. The effects of combining fusion imaging, low-frequency pulsed fluoroscopy, and low-concentration contrast agent during endovascular aneurysm repair. J Vasc Surg. 2016 May;63(5):1147-55.

6. McNally MM, Scali ST, Feezor RJ, Neal D, Huber TS, Beck AW. Three-dimensional fusion computed tomography decreases radiation exposure, procedure time, and contrast use during fenestrated endovascular aortic repair. J Vasc Surg. 2015 Feb;61(2):309-16.

7. Stangenberg L, Shuja F, Carelsen B, Elenbaas T, Wyers MC, Schermerhorn ML. A novel tool for threedimensional roadmapping reduces radiation exposure and contrast agent dose in complex endovascular interventions. J Vasc Surg. 2015 Aug;62(2):448-55.

8. Kauffmann C, Douane F, Therasse E, Lessard S, Elkouri S, Gilbert P, Beaudoin N, Pfister M, Blair JF, Soulez G. Source of errors and accuracy of a two-dimensional/three-dimensional fusion road map for endovascular aneurysm repair of abdominal aortic aneurysm. J Vasc Interv Radiol. 2015 Apr;26(4):544-51.

9. Abi-Jaoudeh N, Glossop N, Dake M, Pritchard WF, Chiesa A, Dreher MR, Tang T, Karanian JW, Wood BJ. Electromagnetic navigation for thoracic aortic stent-graft deployment: a pilot study in swine. J Vasc Interv Radiol. 2010 Jun;21(6):888-95.

10. Valentin J. Avoidance of radiation injuries from medical interventional procedures. Ann ICRP. 2000;30(2):767.

11. Haqqani OP, Agarwal PK, Halin NM, lafrati MD. Defining the radiation "scatter cloud" in the interventional suite. J Vasc Surg. 2013 Nov;58(5):1339-45. 
12. Maurel B, Hertault A, Sobocinski J, Le Roux M, Gonzalez TM, Azzaoui R, Saeed Kilani M, Midulla M, Haulon S. Techniques to reduce radiation and contrast volume during EVAR. J Cardiovasc Surg (Torino). 2014;55(2 Suppl 1):123-31.

13. Resch TA, Törnqvist $P$, Sonesson B, Dias NV. Techniques to reduce radiation for patients and operators during aortic endografting. J Cardiovasc Surg (Torino). 2016 Apr;57(2):178-84.

14. Tepel M, Aspelin P, Lameire N. Contrast-induced nephropathy: a clinical and evidence-based approach. Circulation. 2006;113(14):1799-806.

15. Stacul F, van der Molen AJ, Reimer P, Webb JA, Thomsen HS, Morcos SK, Almén T, Aspelin P, Bellin MF, Clement O, Heinz-Peer G; Contrast Media Safety Committee of European Society of Urogenital Radiology (ESUR). Contrast induced nephropathy: updated ESUR Contrast Media Safety Committee guidelines. Eur Radiol. 2011; 21(12): 2527- 41.

16. Mehta RL, Kellum JA, Shah SV, Molitoris BA, Ronco C, Warnock DG, Levin A; Acute Kidney Injury Network. Acute Kidney Injury Network: report of an initiative to improve outcomes in acute kidney injury. Crit Care 2007;11(2):R31.

17. Haddad F, Greenberg RK, Walker E, Nally J, O'Neill S, Kolin G, Lyden SP, Clair D, Sarac T, Ouriel K. Fenestrated endovascular grafting: The renal side of the story. J Vasc Surg. 2005 Feb;41(2):181-90.

18. Lieberman PL, Seigle RL. Reactions to radiocontrast material: anaphylactoid events in radiology. Clin Rev Allergy Immunol 1999;17:469-96.

19. Kawasaki D, Fujii K, Fukunaga M, Fukuda N, Masuyama T, Ohkubo N, Kato M. Safety and efficacy of carbon dioxide and intravascular ultrasound-guided stenting for renal artery stenosis in patients with chronic renal insufficiency. Angiology. 2015 Mar;66(3):231-6.

20. Spinosa DJ, Kaufmann JA, Hartwell GD. Gadolinium chelates in angiography and interventional radiology: a useful alternative to iodinated contrast media for angiography. Radiology. 2002 May;223(2):319-25; discussion 326-7.

21. Krasznai AG, Sigterman TA, Bouwman LH. Contrast Free Duplex-Assisted EVAR in Patients with Chronic Renal Insufficiency. Ann Vasc Dis. 2014;7(4):426-9.

22. Kaladji A, Dumenil A, Mahé G, Castro M, Cardon A, Lucas A, Haigron P. Safety and accuracy of endovascular aneurysm repair without pre-operative and intra-operative contrast agent. Eur J Vasc Endovasc Surg. 2015 Mar;49(3):255-61.

23. Tozzi P, Marty B, Ruchat P, Ferrari E, Siniscalchi G, von Segesser LK. Endovascular thoracic aortic aneurysm repair without angiography. Innovations (Phila). 2009;4:32-35.

24. Kobeiter H, Nahum J, Becquemin JP. Zero-contrast thoracic endovascular aortic repair using image fusion. Circulation. 2011 Sep 13;124(11):e280-2.

25. Tacher V, Desgranges P, You K, Ridouani F, Marzelle J, Kobeiter H. Feasibility of Three-Dimensional MR Angiography Image Fusion Guidance for Endovascular Abdominal Aortic Aneurysm Repair. J Vasc Interv Radiol. 2016 Feb;27(2):188-93.

26. Koutouzi G, Henrikson O, Roos H, Zachrisson K, Falkenberg M. EVAR Guided by 3D Image Fusion and CO2 DSA: A New Imaging Combination for Patients With Renal Insufficiency. J Endovasc Ther. 2015 Dec;22(6):912-7. 

CHAPTER

Valorization 
The visualization of the human vasculature using X-ray and contrast media has led to unique diagnostic capabilities and treatment options. Minimal invasive procedures are increasingly performed in modern medicine, accompanied by a reduction of open surgical procedures. In the Netherlands and the United States, annually more than 3,200 or 63,000 aortic aneurysm repair procedures are performed today, with approximately $75 \%$ of them being endovascular repairs.

Fluoroscopy and digital subtraction angiography have become two of the most important tools for image-guided interventions. This thesis has shown that use of multimodal image fusion can decrease procedure time as well as iodinated contrast media dose in endovascular procedures. Such a reduction implies large potential benefits for patients undergoing aortic aneurysms repair for their postoperative renal outcome and morbidity. This thesis also explored the translation of fusion technology to other imaging modalities such as in MRA and use in vessel regions with smaller diameters such as peripheral arteries. Many patients who are referred for interventional procedures are at increased risk for contrast-induced kidney injury which can be as high as $30 \%$ in patients with common associated risk factors like diabetes and pre-existing renal impairment. Recommended prophylaxis with $1-1.5 \mathrm{ml} / \mathrm{kg} / \mathrm{hr}$ saline intravenously at least six hours before and after iodinated contrast administration per case induce additional health care costs of about 56 million Euro per year in the Netherlands. In addition to prolonged hospitalization and its' associated costs, the health risk to patients with chronic heart failure due to volume overload is considerable. This thesis intends to promote the benefits of fusion imagining to endovascular surgeons, interventional radiologists and angiosuite technicians. It needs to be broadly recognized that fusion image technology can effectively reduce procedural iodinated contrast media dose or even eliminate application of contrast media when used in combination with other imaging modalities. All state of the art angiosuite systems today are capable of multimodal image coregistration and visualization of hybrid fusion roadmaps. Medical staff involved in fluoroscopy guided interventions should become familiar with multimodal fusion guidance and offer it to patients with renal impairment or other conditions that do not allow iodinated contrast media administration.

The use of X-rays is crucial for endovascular aortic repair, but also associated with certain risks. Radiation exposure to individuals from image-guided procedures has increased substantially, and by some estimates more than 8-fold since 1980. By use of fusion, the medical staff radiation exposure and associated risk of radiation induced injuries can be reduced. Multimodal image fusion might benefit most patients and medical staff if they were translated into a best clinical practice for complex endovascular procedures. 
This thesis offers prospects for future research and products as already partly outlined in the discussion. Besides technology that can detect and visualize deformation of the vascular roadmap, further improvement of the workflow of multimodal fusion visualization is necessary. Ideally for any procedure, the pre-operative MDCT or MR angiography studies of a patient would be automatically displayed to the operator in a segmented, 3D fashion. No more should be needed than selecting the preoperative imaging study and loading it into a workstation connected to the hybrid operating room. Ideally, a completely automated co-registration algorithm based on the live fluoroscopic image and automated motion correction would run before and during the procedure. By these means the entire 3D information of preoperative imaging could be directly visualized and made accessible for every intervention. Since fluoroscopic imaging has been introduced in the last century, several milestone technology improvements have increased use and applicability of this technology for patient care. Interactive hybrid holographic visualization during interventions is possible and likely to happen within the next years. This thesis provides several impulses that support the evolution of fusion guidance for becoming a key technology for minimal invasive treatments. 

Summary 
Multimodal image fusion is an innovative technology where a 3D vascular map from a diagnostic MDCT angiography can be intraoperatively co-displayed to fluoroscopy without the need for additional iodinated contrast application. In this thesis, the use of multimodal image fusion guidance in endovascular complex aortic aneurysm repair was analyzed. Image fusion technology proved to significantly reduce procedure time and procedural iodinated contrast media. Synthesis from previous research and results from this thesis suggest that both reductions are beneficial for postoperative renal function outcome.

Currently the co-registration between the live fluoroscopy and pre-acquired MDCT volume is operated in a rigid manner. This can lead to fusion image overlay mismatch which has been demonstrated to be mainly due to respiration related vessel displacement as well as temporary straightening of elongated vessel segments during introduction of stiff devices. In an analysis and quantification of respiratory movement of the aorta and side branches, significant movements in the ascending aorta and branch vessels were demonstrated. These movements decrease significantly towards the descending aorta. The origins of the abdominal side branches barely move which emphasizes that image fusion is a valuable tool for thoraco-abdominal fenestrated and branched repair, but fusion might potentially be less accurate in aortic arch repair procedures.

Dataset matching between the MDCT and fluoroscopy is facilitated by means of an intraoperative abdominal $\mathrm{CBCT}$. The additional radiation exposure to the patient was analyzed to be approximately $4 \mathrm{mSv}$, which is about half of the dose of an abdominal MDCT. Attributed risk of lethal cancer due to this additional radiation is low. The radiation exposure to physicians and other staff members does not increase by using CBCT but the medical staff doses are significantly higher during complex endovascular repair compared to standard endovascular repair. Multimodal image fusion has led to a decrease in fluoroscopy time of on average 9 minutes per procedure. Fusion technology is likely to establish as an effective technique to significantly reduce radiation dose for patients and medical staff in complex interventions.

Finally, in a pilot study the feasibility of image fusion using MRA and fluoroscopy for 3D mapping in peripheral artery interventions showed potential for future application of multimodal image fusion for image-guided interventions in smaller vessels and peripheral body parts. This might offer new possibilities for management of image-guided interventions in patients with renal impairment or allergy to iodinated contrast media. 
Samenvatting 


\section{SAMENVATTING}

Multimodale beeldfusie is een innovatieve techniek waarbij 3D informatie van de vaatboom verkregen uit een diagnostische multidectector CT (MDCT) angiografie wordt geprojecteerd over peroperatief verkregen doorlichtingsbeelden zonder toediening van additionele jodiumhoudende contrastmiddelen. In dit proefschrift wordt het gebruik van multimodale beeldfusie bij endovasculaire behandeling van complexe aorta aneurysmata geanalyseerd. Beeldfusietechnologie bleek tot aanzienlijke vermindering van proceduretijd en contrastmiddelgebruik te leiden. Uit eerder onderzoek en uit de resultaten van dit proefschrift blijkt dat beide reducties gunstig zijn voor postoperatieve nierfunctie.

Momenteel is de co-registratie van 'live' doorlichtingsbeelden en vooraf verworven MDCT volume een statisch proces. Dit kan leiden tot discrepantie tussen de gefuseerde beelden. Deze discrepantie ontstaat voornamelijk door ademhalings gerelateerde verplaatsing van de vaatboom tijdens doorlichting en door het tijdelijke strekken van vaatsegmenten tijdens het inbrengen van de rigide voerdraden en endoprothesen. Analyse van de ademhalings gerelateerde beweging van de aorta en haar zijtakken toont aanzienlijke bewegingen in de aorta ascendens, aortaboog en haar plaatselijke zijtakken. Deze beweging neemt duidelijk af in de thoracale aorta descendens, terwijl de oorsprong van de abdominale zijtakken nauwelijks meer bewogen. Uit deze bevindingen kan geconcludeerd worden dat beeldfusie een waardevol instrument kan zijn tijdens de endovasculaire behandeling met thoracoabdominale gefenestreerde en branched endoprothesen. Echter, bij procedures in de aortaboog is het minder zinvol door de grotere discrepantie van fusiebeelden.

Dataset matching tussen de MDCT en doorlichting wordt vergemakkelijkt door een peroperatief gemaakte abdominale cone beam CT (CBCT). De extra stralingsdosis nodig voor de CBCT bleek ca. $4 \mathrm{mSv}$. te zijn, hetgeen overeenkomt met ongeveer de helft van de stralingsdosis van een abdominale MDCT. Het extra risico op mortaliteit door kanker, dat kan worden toegeschreven aan deze extra stralingsdosis is laag. De blootstelling aan extra röntgenstraling van artsen en andere medewerkers neemt niet toe door het gebruik van $\mathrm{CBCT}$, maar de totale stralingsdosis voor het behandelteam is significant hoger tijdens endovasculaire behandelingen van complexe aneurysmata van de aorta in vergelijking met die van standaard abdominale en thoracale aorta aneurysmata. Multimodale beeldfusie heeft geleid tot een daling van de doorlichtingstijd van gemiddeld 9 minuten per procedure. Fusietechnologie ontpopt zich als een effectieve techniek om een aanzienlijke vermindering in stralingsdosis voor patiënten en medisch personeel te bewerkstelligen tijdens complexe endovasculaire interventies.

Tot slot, wordt in een pilotstudie naar de haalbaarheid van de beeldfusie tussen MRA en doorlichting in perifere arteriële interventies aangetoond dat de techniek zich ook leent 
voor toekomstige interventies in kleinere bloedvaten in perifere lichaamsdelen. Deze techniek biedt mogelijkheden voor percutane interventies bij patiënten met nierinsufficiëntie en allergie voor jodiumhoudende contrastmiddelen. 

Acknowledgement 
This thesis is the result of clinical research carried out during my residency at Maastricht University Medical Center. It has come together under the guidance of my respected PhD advisors, the counsel and support of my dear colleagues, friends and family as well as the consent of the patients involved in our research and the positive evaluation of the PhD thesis commission. The work on this thesis has enriched my early career, left me with many colleagues turning to friends, and helped me become the physician I am today. While I am grateful for every single one that has accompanied me on this journey, I would like to thank some in particular.

Prof. Dr. Schurink, dear Geert Willem, for introducing me to the field of endovascular and open vascular surgery, for your guidance and care in- and outside the operating theatre. I could not have wished for a better PhD advisor as you have given me the freedom to develop my own field of research while gently supervising me and supporting me with your counsel and time every step of the way. I very much enjoyed the conversations we had and look forward to many more in the future.

Prof. Dr. de Haan, dear Michiel, for guiding my residency training as training supervisor and my PhD as an advisor and for allowing me to explore new opportunities that I came upon during this time. Your shared passion for Interventional Radiology made me discover my own passion for our field which laid the groundwork for this thesis.

Dr. Jeukens, dear Cécile, for your guidance and continuous support as my PhD co-advisor. Your profound technical knowledge has leveraged the quality of the presented research. I am very grateful for the time we shared working on this project, which led to new exciting ventures derived from the research of this thesis.

Dr. Nelemans, dear Patty, your expertise in statistical data analysis, and profound knowledge in medical research not only left a footprint in this thesis but also made me become a better researcher. I very much enjoyed the many times and hours we spent together, especially when you visited me in Stanford. Dear Patty, thank you for your friendship, your beautiful character and the great work you have put into my projects.

Dr. van Zwam, dear Wim, I was very honored when you chose me to be your paranymph for your thesis. You are my role model as an interventional radiologist and researcher. I deeply value your opinion in medicine, science and on life in general. You have always been there for me and always supported my ambitions. I felt that together we were one 
four-handed interventional radiologist, and I truly miss the time with you in the angiosuite. I am grateful to call you my friend and feel very honored that you have agreed to return the favor as my paranymph.

Prof. Dr. Wildberger, dear Joachim, as head of the department of radiology you have overlooked my professional and personal development and helped me to evolve as a radiologist and clinical researcher. I will not forget your many great advices and especially the unconditional support you gave me in difficult times.

Dr. Das, dear Marco, for all your trust, guidance and the many tips and tricks I have learned from you in the angiosuite. You are a great teacher and perfect example of how to achieve high professionalism in research and clinical work. Your introducing me to the Cardiovascular and Interventional Society of Europe has significantly contributed to achieving impact with my research.

The interventional radiology technicians for your support during my interventional radiology training and for the fun times we had throughout my years of residency. I will remember these years of training joyfully. Thank you for your great assistance with my research projects in the angiosuite. Without your help, none of these projects could have been achieved. Especially I would like to thank Geert, Pascal, Paul, Jan, Ellen, Ankie, Judith, Dionne, Cindy, Peter and Chris.

Prof. Dr. Beets-Tan, dear Regina, for being a mentor and close friend on whose counsel and advice I can always rely for my professional and my private life. Your experience and outlook on life together with the similarities and values we share have helped me make decisions that contributed to where I am right now. Thank you for your love and support.

The CT technicians and the IT team, especially Jef, Ralf, Hans, Roy, Lars, Marcel, Sander and Frans, for your prompt technical support whenever I needed it.

My dear colleagues from the department of Radiology, especially Prof. Robben, dear Simon, Prof. Hofmann, dear Paul, and my dear colleagues from the department of Vascular Surgery, for training me and accompanying my residency, making it a time I will always cherish. 
Dear Stella, for your support and friendship inside and outside the hospital.

Dear Maud, Miguel, Elleke, Marjolein, Daan, Doenja, Max, Casper, Bart, and Rene for being the best peers I could imagine and for enriching my residency with friendship, love and laughter. Our group was unique! You have a special place in my heart and I look back with a big smile on the great times and moments we shared.

My dear parents, siblings and in-laws for reminding me that there is a life and values besides career.

My dearest Oliver, for your unconditional love and support that empower me to pursue my dreams and lead the life I envision. You are a true gentleman; I admire your unrivaled strength and kindness. ...As long as there are stars above you, and longer if I can. 
Curriculum Vitae 


\section{CURRICULUM VITAE}

Anna-Margaretha Hedwig Sailer was born on $20^{\text {th }}$ of November 1984 in Ulm, Germany. Following her high school graduation, she pursued her medical studies at RWTH Aachen University with clinical internships in Brisbane, Sydney and Maastricht. Anna additionally enrolled in a Master's of Economics program between RWTH Aachen University and Tsinghua University Beijing. During her semester in Beijing, she took lessons in traditional Chinese medicine at the Institute of Chinese Materia Medica. She was granted scholarships by McKinsey \& Company global leadership program 'Capstone' and Aachen University female leadership program 'Tandem'. During a 10-week internship at the German Federal Parliament she was involved in developing the 2010 reform of the German healthcare system. In 2010, she concluded her studies with a degree as Medical Doctor (MD), a Master of Business Administration (MBA) and the defense of her German Doctoral thesis (Dr. med.).

During her clinical career as a resident radiology at Maastricht University Medical Center Anna specialized in vascular and interventional radiology. She spent the third year of her residency in The Hague at MCH Westeinde, and in 2015 Anna spent eight month as a research fellow at Stanford University Medical Center where she now continues her professional career. She received a personal funding by the Koninklijke Nederlandse Akademie van Wetenschappen (KNAW) and a Seed Grant from the Stanford Cardiovascular Institue. She initiated her PhD in 2013 under the guidance of Prof. Geert Willem Schurink and Prof. Michiel de Haan and Co-promotor Dr. Cécile Jeukens. Anna became a board-certified radiologist in April 2016. She is a faculty member of CIRSE and RSNA and is married to Wilhelm Oliver Karmann. They live in Stanford and Los Angeles. 
List of Publications 


\section{LIST OF PUBLICATIONS AS OF OCTOBER 2016}

This thesis

Sailer AM, de Haan MW, Jacobs MJ, Wildberger JE, Schurink GW. CTA with fluoroscopy image fusion guidance in endovascular complex aortic aneurysm repair. Eur J Vasc Endovasc Surg 2014 Apr;47(4):349-56.

Oral presentations at the European Congress of Radiology (ECR), Vienna 2014, at the Dutch Radiology conference 'Radiologendagen', Hertogenbosch 2013; at the Dutch Vascular Conference 'Vaatdagen', Noordwijkerhout 2014 and the Cardiovascular and Interventional Radiology Society (CIRSE) conference, Glasgow 2014 (invited special focus talk).

Sailer AM, Wagemans BA, Das M, Nelemans PJ, de Haan MW, Wildberger JE, Schurink GW. Quantification of respiratory movement of the aorta and side branches. Journal of Endovasc Ther 2015 Dec;22(6):905-11.

Oral presentation at the Radiological Society of North America (RSNA) conference, Chicago 2014.

Sailer AM, Schurink GWH, de Graaf R, Wildberger JE, van Zwam WH, de Haan MW, Kemerink GJ, Jeukens CRLPN. Radiation exposure of abdominal Cone Beam Computed Tomography. Cardiovasc Interv Radiol 2015 Feb;38(1):112-20.

Oral presentation at the Cardiovascular and Interventional Radiology Society (CIRSE) conference, Barcelona 2014.

Sailer AM, Schurink GWH, Bol ME, de Haan MW, van Zwam WH, Wildberger JE, Jeukens CRLPN. Occupational radiation exposure during endovascular aortic repair. Cardiovasc Interv Radiol 2015 Aug;38(4):827-32.

Oral presentation at the Radiological Society of North America (RSNA) conference, Chicago 2014.

Sailer AM, Nelemans PJ, van Berlo C, Yazar O, de Haan MW, Fleischmann D, Schurink GW. Endovascular repair of complex aortic aneurysms: Prevalence of Acute Kidney Injury and effect on long term renal function. Eur Radiol 2016 Jun;26(6):1613-9.

Oral presentation at the Radiological Society of North America (RSNA) conference, Chicago 2015.

Sailer AM, de Haan MW, de Graaf R, van Zwam WH, Schurink GWH, Nelemans PJ, Wildberger JE, Das M. Fusion image guidance in endovascular peripheral artery interventions - a feasibility study. Cardiovasc Interv Radiol 2015 Apr;38(2):314-21.

Oral presentation at the European Congress of Radiology (ECR), Vienna 2013. 
Other

Stoffels I, Wolter TP, Sailer AM, Pallua N. The impact of silicone spray on scar formation. A single-center placebo-controlled double-blind trial. Hautarzt 2010;61:332-8. [article in German].

Sailer AM, van Ommen VG, Tordoir JH, Schurink GW, van Zwam WH. latrogenic type A aortic dissection: conservative treatment after complicated left subclavian artery recanalization. J Vasc Interv Radiol 2013 Dec;24(12):1923-5.

Sailer AM, Grutters JP, Wildberger JE, Hofman PA, Wilmink JT, van Zwam WH. Costeffectiveness of CTA, MRA and DSA in patients with non-traumatic subarachnoid haemorrhage. Insights Imaging 2013 Aug;4(4):499-507.

Sailer AM, Wagemans BA, Nelemans PJ, de Graaf R, van Zwam WH. Diagnosing intracranial aneurysms with MR Angiography: Systematic review and meta-analysis. Stroke 2014 Jan;45(1):119-26.

Sailer AM, Douwes DC, Cappendijk VC, Bakers FC, Wagemans BA, Wildberger JE, Kessels AG, Beets-Tan RG. RECIST measurements in cancer treatment: Is there a role for physician assistants? Cancer Imaging 2014 Apr;14(1):12.

de Graaf R, Jalaie H, Sailer AM. Rekanalisation des benignen Venenverschlusses. Bildgebung, Indikation \& Behandlung. Interventionelle Radiologie Scan 2014; 02(03): 235250. DOI: 10.1055/s-0034-1377583 [article in German].

de Graaf R, van Laanen J, Sailer A, Tordoir J. Long segment recanalization and dedicated central venous stenting in an ultimate attempt to restore vascular access central vein outflow. J Vasc Access. 2014;15 Suppl 7:109-13.

Jalaie H, Grommes J, Sailer A, Binnebösel M, Kalder J, Greiner A, Schurink GWH, Jacobs MJ. Treatment of symptomatic aberrant subclavian arteries. Eur J Vasc Endovasc Surg 2014 Nov;48(5):521-6.

de Graaf R, de Wolf M, Sailer AM, van Laanen J, Wittens C, Jalaie H. Iliocaval confluence stenting for chronic venous occlusions. Cardiovasc Interv Radiol 2015 Oct;38(5):1198-204.

Seehofnerová A, Kok M, Mihl C, Douwes D, Sailer A, Nijssen E, de Haan MW, Wildberger $\mathrm{JE}$, Das M. Feasibility of low contrast media volume in CT angiography of the aorta. Eur Journal of Radiology Open 2015;(2):58-65. 
Sailer AM, van Zwam WH, Wildberger JE, Grutters JP. Cost-effectiveness analysis in diagnostic imaging: A stepwise appraoch. Eur Radiol 2015 Dec;25(12):3629-37.

Kok M, de Haan MW, Mihl C, Hendriks B, Eijsvoogel NG, Sailer AM, Schnerr RS, Schurink GW, Wildberger JE, Das M. Individualized CT angiography protocols for the evaluation of the aorta. J Vasc Interv Radiol 2016 Apr;27(4):531-8.

Mistelbauer G, Schmidt J, Sailer AM, Baeumler K, Fleischmann D. Aortic dissection maps: Comprehensive visualization of aortic dissections for risk assesment. in: Bruckner, Preim, Vilanova (eds). Eurographics Workshop on Visual Computing for Biology and Medicine 2016, p1-10.

Sailer AM, Paulis L, Vergoossen L, Schurink GW, Mees B, Das M, Wildberger JE, de Haan MW, Jeukens CRLPN. Real-time patient and staff radiation dose monitoring in IR practice. Cardiovasc Interv Radiol. (accepted for publication). 
